\title{
Statistics of Infima and Stopping Times of Entropy Production and Applications to Active Molecular Processes
}

\author{
Izaak Neri, ${ }^{1,2,3, *}$ Édgar Roldán, ${ }^{1,3,4, \dagger}$ and Frank Jülicher ${ }^{1,3, \$}$ \\ ${ }^{1}$ Max Planck Institute for the Physics of Complex Systems, Nöthnitzer Straße 38, 01187 Dresden, Germany \\ ${ }^{2}$ Max Planck Institute of Molecular Cell Biology and Genetics, \\ Pfotenhauerstraße 108, 01307 Dresden, Germany \\ ${ }^{3}$ Center for Advancing Electronics Dresden cfAED, 01062 Dresden, Germany \\ ${ }^{4}$ GISC-Grupo Interdisciplinar de Sistemas Complejos, 28040 Madrid, Spain
}

(Received 14 April 2016; revised manuscript received 22 September 2016; published 21 February 2017)

\begin{abstract}
We study the statistics of infima, stopping times, and passage probabilities of entropy production in nonequilibrium steady states, and we show that they are universal. We consider two examples of stopping times: first-passage times of entropy production and waiting times of stochastic processes, which are the times when a system reaches a given state for the first time. Our main results are as follows: (i) The distribution of the global infimum of entropy production is exponential with mean equal to minus Boltzmann's constant; (ii) we find exact expressions for the passage probabilities of entropy production; (iii) we derive a fluctuation theorem for stopping-time distributions of entropy production. These results have interesting implications for stochastic processes that can be discussed in simple colloidal systems and in active molecular processes. In particular, we show that the timing and statistics of discrete chemical transitions of molecular processes, such as the steps of molecular motors, are governed by the statistics of entropy production. We also show that the extreme-value statistics of active molecular processes are governed by entropy production; for example, we derive a relation between the maximal excursion of a molecular motor against the direction of an external force and the infimum of the corresponding entropy-production fluctuations. Using this relation, we make predictions for the distribution of the maximum backtrack depth of RNA polymerases, which follow from our universal results for entropy-production infima.
\end{abstract}

DOI: 10.1103/PhysRevX.7.011019

Subject Areas: Biological Physics, Statistical Physics

\section{INTRODUCTION AND STATEMENT OF THE MAIN RESULTS}

The total entropy $S_{\text {tot }}(t)$ produced by a mesoscopic process in a finite time interval $[0, t]$ is stochastic, and for a single realization it can be negative because of fluctuations. The second law of thermodynamics implies that its average, taken over many realizations of the process, increases in time, $\left\langle S_{\text {tot }}(t)\right\rangle \geq 0$. In the 19 th century, Maxwell formulated the idea of a stochastic entropy [1], and in the last few decades, definitions of entropy production of nonequilibrium processes were established using the theory of stochastic processes [2-20].

Little is known beyond the second law about the statistics of entropy-production fluctuations. The best insights, so far, in fluctuations of entropy production are provided by fluctuation theorems. They express a fundamental

\footnotetext{
*izaakneri@posteo.net

†edgar@edgarroldan.com

*julicher@pks.mpg.de
}

Published by the American Physical Society under the terms of the Creative Commons Attribution 3.0 License. Further distribution of this work must maintain attribution to the author(s) and the published article's title, journal citation, and DOI. asymmetry of the fluctuations of entropy production: It is exponentially more likely to produce a positive amount of entropy than to reduce entropy by the same but negative amount. An example is the detailed fluctuation theorem, which can be written as $p_{\mathrm{S}}\left(S_{\text {tot }} ; t\right) / p_{\mathrm{S}}\left(-S_{\text {tot }} ; t\right)=e^{S_{\text {tot }} / k_{\mathrm{B}}}$, where $k_{\mathrm{B}}$ is Boltzmann's constant. Here, $p_{\mathrm{S}}\left(S_{\mathrm{tot}} ; t\right)$ is the probability density describing the distribution of the entropy production $S_{\text {tot }}$ at a given time $t$. The detailed fluctuation theorem is universal and holds for a broad class of physical processes in a steady state [3-5,8-10,14,21-26]. Moreover, the detailed fluctuation theorem has been tested in several experiments [27-36]; for reviews, see Refs. [37-39].

In addition to fluctuation theorems, an important question is to understand the extreme-value statistics of entropy production. In particular, because entropy must increase on average, it is interesting to understand the statistics of records of negative entropy production during a given time interval $[0, t]$. To address this question, here we introduce the finite-time infimum of entropy production, $S_{\text {inf }}(t) \equiv \inf _{0 \leq \tau \leq t} S_{\text {tot }}(\tau)$, which is the negative record of entropy production for a single realization of the process over a time interval $[0, t]$.

In this paper, we derive universal equalities and inequalities on the statistics of entropy-production infima. We show that the mean of the finite-time infimum of the 
stochastic entropy production is bounded from below by minus the Boltzmann constant:

$$
\left\langle S_{\text {inf }}(t)\right\rangle \geq-k_{\mathrm{B}} .
$$

This infimum law for entropy production is illustrated in Fig. 1(a) and expresses a fundamental bound on how much entropy can be reduced in a finite time. The infimum law follows from a universal bound for the cumulative distribution of entropy-production infima:

$$
\operatorname{Pr}\left(S_{\text {inf }}(t) \geq-s\right) \geq 1-e^{-s / k_{\mathrm{B}}} .
$$

Here, $\operatorname{Pr}(\cdot)$ denotes the probability of an event, and the lefthand side is the cumulative distribution of entropy production with $s \geq 0$. Remarkably, as we show in this paper, the infimum law, given by Eq. (1), is universal and holds in general for classical and stationary stochastic processes.

The global infimum of entropy production, $S_{\text {inf }}^{\infty} \equiv$ $\lim _{t \rightarrow \infty} S_{\text {inf }}(t)$, is the lowest value that entropy production will ever reach in one realization of the process; note that the global infimum is always smaller than or equal to the finite-time infimum, $S_{\mathrm{inf}}^{\infty} \leq S_{\mathrm{inf}}(t)$. We show that the distribution of the global infimum of entropy production is exponential,

$$
p_{S_{\text {inf }}^{\infty}}(-s)=\frac{e^{-s / k_{\mathrm{B}}}}{k_{\mathrm{B}}},
$$

where $s \geq 0$, and the mean value of the global infimum is equal to minus the Boltzmann constant:

$$
\left\langle S_{\text {inf }}^{\infty}\right\rangle=-k_{\mathrm{B}}
$$

The shape of the distribution of the global infimum implies that the infimum lies with $50 \%$ probability within $-k_{\mathrm{B}} \ln 2 \leq$ $S_{\text {inf }}^{\infty} \leq 0$, and its standard deviation equals the Boltzmann constant. Whereas Eqs. (1) and (2) hold generally in steady states, the equalities given by Eqs. (3) and (4) are shown to be true for continuous stochastic processes.

Related to the global infimum are the passage probabilities $\mathrm{P}_{+}^{(2)}\left(\mathrm{P}_{-}^{(2)}\right)$ for entropy production to reach a threshold $s_{\text {tot }}^{+}\left(-s_{\text {tot }}^{-}\right)$without having reached $-s_{\text {tot }}^{-}\left(s_{\text {tot }}^{+}\right)$ before. This corresponds to the stochastic process $S_{\text {tot }}(t)$ with two absorbing boundaries, a positive absorbing boundary at $S_{\text {tot }}(t)=s_{\text {tot }}^{+}$and a negative absorbing boundary at $S_{\text {tot }}(t)=-s_{\text {tot }}^{-}$. If the process $S_{\text {tot }}(t)$ is continuous and $\left\langle S_{\text {tot }}(t)\right\rangle \neq 0$, we find

$$
\begin{aligned}
& \mathrm{P}_{+}^{(2)}=\frac{e^{s_{\text {tot }}^{-} / k_{\mathrm{B}}}-1}{e^{s_{\mathrm{tot}}^{-} / k_{\mathrm{B}}}-e^{-s_{\mathrm{tot}}^{+} / k_{\mathrm{B}}}}, \\
& \mathrm{P}_{-}^{(2)}=\frac{1-e^{-s_{\mathrm{tot}}^{+} / k_{\mathrm{B}}}}{e^{s_{\mathrm{tot}}^{-} / k_{\mathrm{B}}}-e^{-s_{\mathrm{tot}}^{+} / k_{\mathrm{B}}}} .
\end{aligned}
$$

Interestingly, if $s_{\text {tot }}^{+} \neq s_{\text {tot }}^{-}$, the relations (5) and (6) relate entropy-production fluctuations of events with different amplitudes. The asymptotic value of the passage
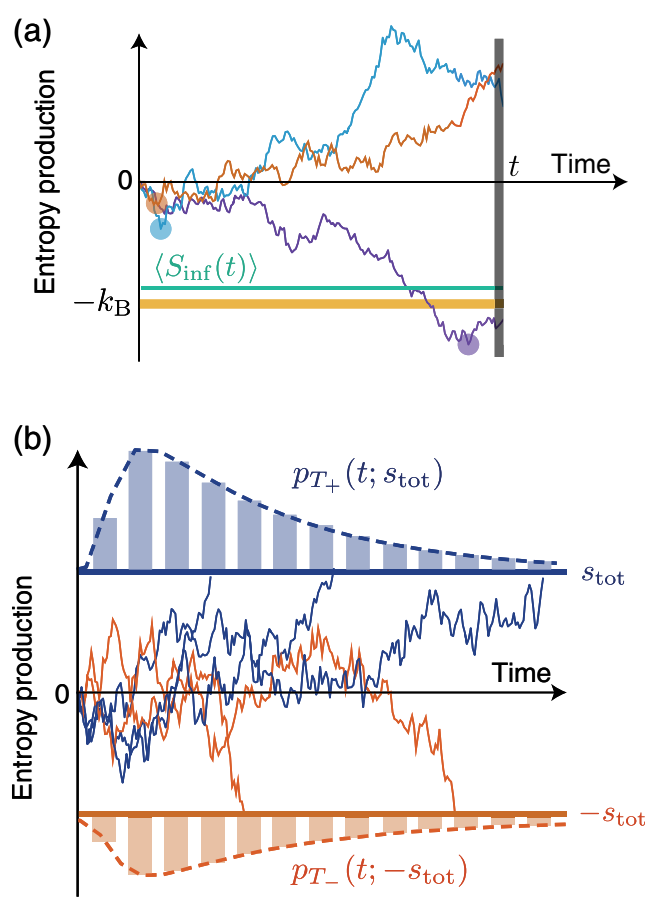

(c)

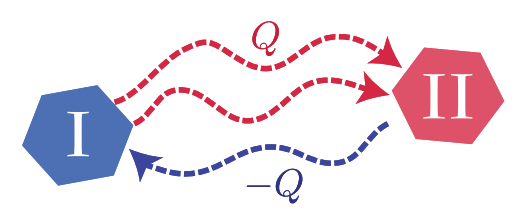

FIG. 1. Illustration of three key results of the paper. (a) Schematic representation of the infimum law for entropy production. Several stochastic trajectories of entropy production are shown (solid lines), and their infima are indicated (filled circles). The infimum law implies that the average infimum of the entropy production (green solid line) is larger than or equal to $-k_{\mathrm{B}}$ (orange line). (b) First-passage-time fluctuation theorem for entropy production with two absorbing boundaries. We show examples of trajectories of stochastic entropy production as a function of time, which first reach the positive threshold $s_{\text {tot }}$ (horizontal thick blue line) and which first reach the negative threshold $-s_{\text {tot }}$ (horizontal thick red line). The probability distribution $p_{T_{+}}\left(t ; s_{\text {tot }}\right)$ to first reach the positive threshold at time $t$ and the probability distribution $p_{T_{-}}\left(t ;-s_{\text {tot }}\right)$ to first reach the negative threshold at time $t$ are related by Eq. (8). (c) Waiting-time fluctuations: The statistics of the waiting times between two states I and II are the same for forward and backward trajectories that absorb or dissipate a certain amount of heat $Q$ in isothermal conditions.

probability $\mathrm{P}_{+}^{(2)}$ for $s_{\text {tot }}^{+}=+\infty$ is the probability that entropy never reaches the value $-s_{\text {tot }}^{-}$. It is equal to the probability that the global infimum is larger than or equal to $-s_{\text {tot }}^{-}$. The relations for the passage probabilities given by Eqs. (5) and (6) thus imply Eqs. (3) and (4) for the global infimum. Notably, the infima and passage statistics of entropy production are independent of the strength of the nonequilibrium driving, i.e., the mean entropyproduction rate. 
We also discuss stopping times. A stopping time is the time when a stochastic process satisfies a certain criterion for the first time. Here, we discuss $s_{\text {tot }}$-stopping times $T_{+}$, for which entropy production at the stopping time equals $S_{\text {tot }}\left(T_{+}\right)=s_{\text {tot }}$, with $s_{\text {tot }}>0$. An example is the firstpassage time of entropy production, at which entropy production reaches $s_{\text {tot }}$ for the first time. This value of entropy $S_{\text {tot }}\left(T_{+}\right)$is a new record of entropy production, and first-passage times of entropy production are thus times at which a given record is reached. Analogously, we define a $\left(-s_{\text {tot }}\right)$-stopping time $T_{-}$associated with $T_{+}$, for which entropy production at the stopping time equals $S_{\text {tot }}\left(T_{-}\right)=$ $-s_{\text {tot }}$. For example, if $T_{+}$is the first-passage time of entropy production to first reach $s_{\text {tot }}$, then $T_{-}$is the first-passage time of entropy production to first reach $-s_{\text {tot }}$. Remarkably, we find that the mean stopping time $\left\langle T_{+}\right\rangle$equals the mean stopping time $\left\langle T_{-}\right\rangle$:

$$
\left\langle T_{+}\right\rangle=\left\langle T_{-}\right\rangle .
$$

A similar equality holds for all the higher-order moments of stopping times of entropy production. These results follow from the stopping-time fluctuation theorem

$$
\frac{p_{T_{+}}\left(t ; s_{\mathrm{tot}}\right)}{p_{T_{-}}\left(t ;-s_{\mathrm{tot}}\right)}=e^{s_{\mathrm{tot}} / k_{\mathrm{B}}}
$$

which we derive in this paper for classical and continuous stochastic processes in a steady state. Here, $p_{T_{+}}\left(t ; s_{\text {tot }}\right)$ is the probability density for the stopping time $T_{+}$, and $p_{T_{-}}\left(t ;-s_{\text {tot }}\right)$ is the probability density for the stopping time $T_{-}$. The stopping-time fluctuation theorem (8) is illustrated in Fig. 1(b) for the example where $T_{+}$and $T_{-}$ are first-passage times of entropy production with two absorbing boundaries.

Other examples of stopping times are waiting times, defined as the time a stochastic trajectory takes while changing from an initial state I to a final state II; see Fig. 1(c). In this paper, we show that for a nonequilibrium and stationary isothermal process, the ratio of waiting-time distributions corresponding to forward trajectories (I $\rightarrow$ II) and backward trajectories (II $\rightarrow$ I) obeys

$$
\frac{p_{T_{+}^{\mathrm{I} \mapsto \mathrm{II}}}(t ;-Q)}{p_{T_{-}^{\mathrm{II}}(t)}(t ; Q)}=e^{-Q / k_{\mathrm{B}} T_{\mathrm{env}}},
$$

for all trajectories between I and II that exchange the amount $Q$ of heat with an equilibrated environment at temperature $\mathrm{T}_{\text {env }}$; if $Q>0$, then the system absorbs heat from the environment. Here, $p_{T_{+}^{\mathrm{I}+\mathrm{II}}}(t ;-Q)$ denotes the probability density for the waiting time $T_{+}^{\mathrm{I} \rightarrow \mathrm{II}}$ to reach state II while absorbing the heat $Q$. Equation (9) is a generalization of the local detailedbalance condition for transition rates $k_{+}^{\mathrm{I} \rightarrow \mathrm{II}} / k_{-}^{\mathrm{I} \rightarrow \mathrm{I}}=e^{-Q / k_{\mathrm{B}} \mathrm{T}_{\text {env }}}$ [40-42]. Indeed, transition rates are given by $k^{-1}=$ $\int_{0}^{\infty} \mathrm{d} t t p_{\mathrm{T}}(t)$. Notably, Eq. (9) implies a symmetry relation on the normalized waiting-time distributions

$$
\frac{p_{T_{+}^{\mathrm{I} \rightarrow \mathrm{II}}}(t \mid-Q)}{\mathrm{P}_{+}^{\mathrm{I} \rightarrow \mathrm{II}}}=\frac{p_{T_{-}^{\mathrm{II} \rightarrow \mathrm{I}}(t \mid Q)}}{\mathrm{P}_{-}^{\mathrm{II} \rightarrow \mathrm{I}}}
$$

where $\mathrm{P}=\int_{0}^{\infty} \mathrm{d} t p_{T}(t)$. Therefore, the mean waiting times $\langle T\rangle=1 /(k \mathrm{P})$ for the forward and backward transitions are the same, $\left\langle T_{+}^{\mathrm{I} \rightarrow \mathrm{II}}\right\rangle=\left\langle T_{-}^{\mathrm{II} \rightarrow \mathrm{I}}\right\rangle$.

We derive all these results on infima, passage probabilities, and stopping times of entropy production in a new unified formalism that uses the theory of martingales $[26,43,44]$, and we apply our results to the dynamics of colloidal particles in periodic potentials and molecular motors, which transduce chemical energy into mechanical work. The paper is structured as follows: In Sec. II, we briefly review the formalism of stochastic thermodynamics. In Sec. III, we discuss the connection between martingale processes and entropy production. In Secs. IV-VI, we derive, respectively, the infimum law (1) and the bound (2); the statistics of the global infimum of entropy production (3) and (4) and the equalities for the passage probabilities (5) and (6); and fluctuation theorems for stopping times of entropy production, which include first-passage times of entropy production (8) and waiting times of stochastic processes (9). We apply our results in Sec. VII to a drifted colloidal particle moving in a periodic potential. In Sec. VIII, we apply our results to discrete molecular processes such as the stepping statistics of molecular motors or the dynamics of enzymatic reactions. The paper concludes with a discussion in Sec. IX.

\section{STOCHASTIC THERMODYNAMICS AND ENTROPY PRODUCTION}

We first briefly review the basic concepts of stochastic entropy production for discrete processes based on path probabilities. Discrete processes include processes in discrete time (e.g., Markov chains) and processes in continuous time with discrete states (e.g., Markov jump processes). We then present a measure-theoretic formalism of stochastic thermodynamics, which defines entropy production for discrete and continuous processes (e.g., Langevin processes).

\section{A. Entropy production for discrete processes}

We consider the dynamics of a mesoscopic system in a nonequilibrium steady state and describe its dynamics with the coarse-grained state variables $\omega(t)=\left(\boldsymbol{q}(t), \boldsymbol{q}^{*}(t)\right)$ at time $t$. The variables $\boldsymbol{q}(t)$ represent $n$ degrees of freedom that are even under time reversal, and the variables $\boldsymbol{q}^{*}(t)$ represent $n^{*}$ degrees of freedom that are odd under time reversal [45]. Notably, the variables $\boldsymbol{q}(t)$ and $\boldsymbol{q}^{*}(t)$ represent the dynamics of collective modes in a system of interacting particles; for instance, $\boldsymbol{q}(t)$ describes the position of a colloidal particle in a fluid and $\boldsymbol{q}^{*}(t)$ its effective momentum.

In a given time window $[0, t]$, the coordinates $\omega(t)$ trace a path in phase space $\omega_{0}^{t}=\{\omega(\tau)\}_{0 \leq \tau \leq t}$. We associate with each trajectory $\omega_{0}^{t}$ a probability density $\mathcal{P}\left(\omega_{0}^{t} ; p_{\text {init }}\right)$, which 
captures the limited information provided by the coarsegrained variables $\omega$, and the fact that the exact microstate is not known; the distribution $p_{\text {init }}$ is the probability density of the initial state $\omega(0)$. We define the entropy production associated with a path $\omega_{0}^{t}$ of a stationary process by [7-9,11]

$$
S_{\text {tot }}(t) \equiv k_{\mathrm{B}} \ln \frac{\mathcal{P}\left(\omega_{0}^{t} ; p_{\mathrm{ss}}\right)}{\mathcal{P}\left(\Theta_{t} \omega_{0}^{t} ; \tilde{p}_{\mathrm{ss}}\right)},
$$

where $\Theta_{t} \omega_{0}^{t}=\{\tilde{\omega}(t-\tau)\}_{\tau=0}^{t}$ is the time-reversed trajectory with $\tilde{\omega}(\tau)=\left(\boldsymbol{q}(\tau),-\boldsymbol{q}^{*}(\tau)\right), p_{\mathrm{ss}}$ is the steady-state distribution in the forward dynamics, and $\tilde{p}_{\mathrm{ss}}$ is the steady-state distribution in the backward dynamics. The ensemble average of entropy production of a stationary process can be expressed as an integral:

$$
\left\langle S_{\text {tot }}(t)\right\rangle=k_{\mathrm{B}} \int \mathcal{D} \omega_{0}^{t} \mathcal{P}\left(\omega_{0}^{t} ; p_{\mathrm{ss}}\right) \ln \frac{\mathcal{P}\left(\omega_{0}^{t} ; p_{\mathrm{ss}}\right)}{\mathcal{P}\left(\Theta \omega_{0}^{t} ; \tilde{p}_{\mathrm{ss}}\right)} .
$$

Here, entropy production is therefore the observable that quantifies time irreversibility of mesoscopic trajectories [46]. In fact, by measuring entropy production, an observer can determine within a minimal time whether a movie of a stochastic process is run forwards or backwards [47].

Microscopic time-reversibility implies that a mesoscopic system in contact with an equilibrium reservoir, or consecutively in contact with different equilibrium reservoirs, satisfies local detailed balance [8,48-50]. Local detailed balance manifests itself in a condition on the path probabilities conditioned on the initial state and reads

$$
\frac{\mathcal{P}\left(\omega_{0}^{t} \mid \omega(0)\right)}{\mathcal{P}\left(\Theta_{t} \omega_{0}^{t} \mid \tilde{\omega}(t)\right)}=e^{S_{\mathrm{env}}(t) / k_{\mathrm{B}}},
$$

where $S_{\text {env }}(t)$ is the entropy change in the environment. If local detailed balance holds, then our definition of entropy production (11) equals the total entropy change, i.e., the sum of the system-entropy change $\Delta S_{\text {sys }}$ [14] and the environment-entropy change $S_{\text {env }}$ :

$$
S_{\mathrm{tot}}(t)=\Delta S_{\mathrm{sys}}(t)+S_{\mathrm{env}}(t),
$$

with

$$
\Delta S_{\mathrm{sys}}(t)=-k_{\mathrm{B}} \ln \frac{p_{\mathrm{ss}}(\omega(t))}{p_{\mathrm{ss}}(\omega(0))} .
$$

Notice that in Eq. (15), we have used $\tilde{p}_{\text {ss }}(\tilde{\omega}(t))=$ $p_{\mathrm{ss}}(\omega(t))[11]$. For systems in contact with one or several thermal baths, the environment-entropy change is related to the heat exchanged between the system and the environment [51]. For systems that violate the local detailed balance condition (13), a physical interpretation of the entropy production (11) in terms of heat exchange is not so direct. Nevertheless, entropy production (11) can always be interpreted as the functional that characterizes timeirreversibility.

\section{B. Entropy production for continuous processes that may have jumps}

For discrete processes, the expressions Eqs. (11) and (12) for the stochastic and the average entropy production are well defined. However, for Langevin processes the path probabilities densities $\mathcal{P}$ are not normalizable. In order to avoid this problem, we use a formalism based on measure theory to define entropy production for continuous processes [43,44,53-56]. Note that this formalism also applies to processes with continuous variables that may undergo discrete jumps [57].

Measure theory studies probabilities of events in terms of a probability space $(\Omega, \mathcal{F}, \mathbb{P})$. The set $\Omega$ of all trajectories $\omega$ is called the sample space, the set $\mathcal{F}$ of all measurable subsets $\Phi$ of $\Omega$ is a $\sigma$-algebra, and the function $\mathbb{P}$ is a measure, which associates probabilities with the measurable subsets $\Phi$. In the following, we identify the symbol $\omega$ with the full trajectory of state variables over all times, $\omega=\left\{\boldsymbol{q}(\tau), \boldsymbol{q}^{*}(\tau)\right\}_{\tau \in(-\infty, \infty)}$.

The concept of a probability measure $\mathbb{P}(\Phi)$ generalizes the path probability densities $\mathcal{P}(\omega)$. The value $\mathbb{P}(\Phi)$ denotes the probability to observe a trajectory $\omega$ in the set $\Phi$; in other words, $\mathbb{P}(\Phi)=\operatorname{Pr}(\omega \in \Phi)$. An example of a measure is

$$
\mathbb{P}(\Phi)=\int_{\boldsymbol{x} \in \Phi} \mathrm{d} \lambda p(\boldsymbol{x}),
$$

where $p(\boldsymbol{x})$ is a probability density of elements $\boldsymbol{x}$ in $\mathbb{R}^{n}$. Here, $\lambda$ denotes the Lebesgue measure, and the Lebesgue integral is over the set $\Phi$. One can also define a probability density $\mathcal{R}(\omega)$ of a measure $\mathbb{P}(\Phi)=\int_{\omega \in \Phi} \mathrm{d} \mathbb{P}$ with respect to a second probability measure $\mathbb{Q}(\Phi)$ using the RadonNikodým theorem [54]

$$
\mathbb{P}(\Phi)=\int_{\omega \in \Phi} \mathrm{d} \mathbb{Q} \mathcal{R}(\omega),
$$

where the integral is over the measurable set $\Phi$ and with respect to the probability space $(\Omega, \mathcal{F}, \mathbb{Q})[54,56]$. The function $\mathcal{R}(\omega)$ is called the Radon-Nikodým derivative, which we denote by $\mathcal{R}(\omega)=\frac{\mathrm{d} \mathbb{P}}{\mathrm{d}}(\omega)$. In Eq. (17), the function $\mathcal{R}(\omega)$ is a generalization of the concept of a probability density $p(\boldsymbol{x})$, which also applies to spaces for which the Lebesgue measure does not exist, e.g., the Wiener space of trajectories of a Brownian particle.

We now consider probability measures of steady-state processes. A stationary probability measure is timetranslation invariant and satisfies $\mathbb{P}=\mathbb{P} \circ \mathrm{T}_{t}$, where $\mathrm{T}_{t}$ is the map that translates a trajectory $\omega$ by a time $t$ as $\boldsymbol{q}(\tau) \rightarrow$ $\boldsymbol{q}(\tau+t)$ and $\boldsymbol{q}^{*}(\tau) \rightarrow \boldsymbol{q}^{*}(\tau+t)$.

A stochastic process $X(\omega ; t)$ provides the value of an observable $X$ at time $t$ for a given trajectory $\omega$. We denote the average or expectation value of $X(\omega ; t)$ by $\langle X(\omega ; t)\rangle_{\mathbb{P}}=$ $\int_{\omega \in \Omega} X(\omega ; t) \mathrm{d} \mathbb{P}$. In the following, a stochastic process 
$X(\omega ; t)$ is sometimes simply denoted by $X(t)$ and its average by $\langle X(t)\rangle$.

Entropy production $S_{\text {tot }}(\omega ; t)$ is an example of a stochastic process. An appropriate definition of entropy production, which generalizes Eq. (11) and applies also to continuous-time processes, can be written using the Radon-Nikodým derivative

$$
S_{\text {tot }}(\omega ; t) \equiv k_{\mathrm{B}} \ln \frac{\left.\mathrm{d} \mathbb{P}\right|_{\mathcal{F}(t)}}{\left.\mathrm{d}(\mathbb{P} \circ \Theta)\right|_{\mathcal{F}(t)}}(\omega)
$$

of the measure $\left.\mathbb{P}\right|_{\mathcal{F}(t)}$ with respect to the time-reversed measure $\left.(\mathbb{P} \circ \Theta)\right|_{\mathcal{F}(t)}[58]$. Here, $\left.\mathbb{P}\right|_{\mathcal{F}(t)}$ denotes the restriction of the measure $\mathbb{P}$ over those events in the sub- $\sigma$-algebra $\mathcal{F}(t) \subset \mathcal{F}$ that is generated by trajectories $\omega_{0}^{t}$ in the time interval $[0, t]$. The time-reversed measure $\mathbb{P} \circ \Theta$ is defined using the time-reversal map $\Theta$, which time reverses trajectories $\omega$ as $\boldsymbol{q}(t) \rightarrow \boldsymbol{q}(-t)$ and $\boldsymbol{q}^{*}(t) \rightarrow-\boldsymbol{q}^{*}(-t)$. Note that Eq. (18) is well defined for continuous-time processes that may contain jumps.

\section{MARTINGALE THEORY FOR ENTROPY PRODUCTION}

A fundamental, but still unexplored, property of entropy production is that in a steady state, its exponential $e^{-S_{\text {tot }}(t) / k_{\mathrm{B}}}$ is a positive and uniformly integrable martingale process. A process is called martingale if its expected value at any time $t$ equals its value at a previous time $\tau$, when the expected value is conditioned on observations up to the time $\tau$ (see Appendix A). Therefore, martingales have zero drift. Martingale processes represent fair games and are widely studied in quantitative finance [59,60], but their use for stochastic thermodynamics has not been explored much [26].

Here, we show that the process $e^{-S_{\text {tot }}(t) / k_{\mathrm{B}}}$ is a martingale (see Appendix B) and thus obeys

$$
\left\langle e^{-S_{\mathrm{tot}}(t) / k_{\mathrm{B}}} \mid \omega_{0}^{\tau}\right\rangle=e^{-S_{\mathrm{tot}}(\tau) / k_{\mathrm{B}}},
$$

for $t>\tau$, where the average is conditioned on a particular trajectory $\omega\left(t^{\prime}\right)$ from $t^{\prime}=0$ up to time $\tau$. From Eq. (19), it follows that martingale processes have a time-independent average. Interestingly, for $e^{-S_{\text {tot }}(t) / k_{\mathrm{B}}}$, this implies the integral fluctuation theorem. Indeed, using Eq. (19) for $\tau=0$ and $S_{\text {tot }}(0)=0$, it follows that $\left\langle e^{-S_{\text {tot }}(t) / k_{\mathrm{B}}}\right\rangle=1$, for arbitrary initial conditions $[2,8,14,61]$.

On average, the total entropy $S_{\text {tot }}(t)$ always increases, and therefore, it cannot be a martingale. However, entropy production is a submartingale, with the property

$$
\left\langle S_{\mathrm{tot}}(t) \mid \omega_{0}^{\tau}\right\rangle \geq S_{\mathrm{tot}}(\tau) .
$$

Equation (20) follows from Eq. (19) and the fact that $e^{-S_{\text {tot }}(t) / k_{\mathrm{B}}}$ is a convex function of $S_{\text {tot }}(t)$. From Eq. (20), it follows that the average entropy production is greater than or equal to zero for any initial condition. Note that this statement is stronger than $\left\langle S_{\text {tot }}(t)\right\rangle \geq 0$, where the brackets denote the steady-state ensemble.

A key property of martingales is Doob's maximal inequality (see Appendix A) $[43,44]$. For $e^{-S_{\text {tot }}(t) / k_{\mathrm{B}}}$, this inequality provides a bound on the cumulative distribution of its supremum:

$$
\operatorname{Pr}\left(\sup _{\tau \in[0, t]}\left\{e^{-S_{\text {tot }}(\tau) / k_{\mathrm{B}}}\right\} \geq \lambda\right) \leq \frac{1}{\lambda}\left\langle e^{-S_{\text {tot }}(t) / k_{\mathrm{B}}}\right\rangle .
$$

Equation (21) is a stronger condition than the well-known Markov inequality Eq. (A7), and it holds for steady-state processes in discrete time and steady-state continuous-time processes with jumps.

Another key property of martingales is Doob's optional sampling theorem. For entropy production, this theorem generalizes Eq. (19) to averages conditioned on stochastic stopping times $T<t$ (see Appendix A):

$$
\left\langle e^{-S_{\mathrm{tot}}(t) / k_{\mathrm{B}}} \mid S_{\mathrm{tot}}(T)\right\rangle=e^{-S_{\mathrm{tot}}(T) / k_{\mathrm{B}}} .
$$

The stopping time $T=T(\omega)$ is the time at which a trajectory $\omega$ satisfies a certain criterion for the first time, and therefore differs for each realization $\omega$; this is a generalization of passage times. Equation (22) holds for steady-state discrete-time processes and for steady-state continuous-time processes with jumps. Equation (22) implies that the expected value of $e^{-S_{\text {tot }}(t) / k_{\mathrm{B}}}$, over all trajectories for which the value of entropy at the stochastic stopping time $T$ (with $T<t$ ) is given by the value $s_{\text {tot }}$, equals $e^{-s_{\text {tot }} / k_{\mathrm{B}}}$.

\section{INFIMUM LAW}

Using the martingale property of $e^{-S_{\mathrm{tot}}(t) / k_{\mathrm{B}}}$, we derive the infimum law for entropy production, which holds for nonequilibrium stationary processes. From Eq. (21) and the integral fluctuation theorem, $\left\langle e^{-S_{\text {tot }}(t) / k_{\mathrm{B}}}\right\rangle=1$, we find the following bound for the cumulative distribution of the supremum of $e^{-S_{\mathrm{tot}}(t) / k_{\mathrm{B}}}$,

$$
\operatorname{Pr}\left(\sup _{\tau \in[0, t]}\left\{e^{-S_{\mathrm{tot}}(\tau) / k_{\mathrm{B}}}\right\} \geq \lambda\right) \leq \frac{1}{\lambda},
$$

for $\lambda \geq 0$. Equation (23) implies a lower bound on the cumulative distribution of the infimum of $S_{\text {tot }}$ in a given time interval $[0, t]$ :

$$
\operatorname{Pr}\left(\frac{S_{\text {inf }}(t)}{k_{\mathrm{B}}} \geq-s\right) \geq 1-e^{-s},
$$

where

$$
S_{\text {inf }}(t) \equiv \inf _{\tau \in[0, t]} S_{\text {tot }}(\tau)
$$


is the finite-time infimum and $s \geq 0$. The right-hand side of Eq. (24) is the cumulative distribution of an exponential random variable $S$ with distribution function $p_{S}(s)=e^{-s}$. From Eq. (24), it thus follows that the random variable $-S_{\text {inf }}(t) / k_{\mathrm{B}}$ dominates stochastically over $S$, and this implies an inequality in the mean values of the corresponding random variables, as we show in Appendix C. From Eq. (24), we thus find the following universal bound for the mean infimum of entropy production:

$$
\left\langle S_{\text {inf }}(t)\right\rangle \geq-k_{\mathrm{B}} .
$$

The infimum law given by Eq. (26) holds for stationary stochastic processes in discrete time and for stationary stochastic processes in continuous time for which $e^{-S_{\text {tot }}(t) / k_{\mathrm{B}}}$ is right continuous.

For the special case of isothermal processes, the total entropy change $S_{\text {tot }}(t)=\Delta S_{\text {sys }}(t)-Q(t) / \mathrm{T}_{\text {env }}=\Delta S_{\text {sys }}(t)+$ $[W(t)-\Delta E(t)] / \mathrm{T}_{\mathrm{env}}$, with $Q(t)$ denoting the heat absorbed by the system from the thermal reservoir, $W(t)$ denoting the work done on the system, and $\Delta E(t)$ denoting the internal energy change of the system. We thus have a bound on the infimum of the dissipated part of the work $W^{\text {diss }}$, which reads

$$
\left\langle W_{\mathrm{inf}}^{\text {diss }}(t)\right\rangle \geq-k_{\mathrm{B}} \mathrm{T}_{\text {env }} .
$$

Here, we have defined the dissipated work $W^{\text {diss }}(t)=$ $W(t)-\Delta F(t)$, with $\Delta F(t)=\Delta E(t)-\mathrm{T}_{\text {env }} \Delta S_{\text {sys }}(t)$. For an isothermal process for which all states have the same energy and entropy, we have $\Delta F(t)=0$, and thus

$$
\left\langle W_{\text {inf }}(t)\right\rangle \geq-k_{\mathrm{B}} \mathrm{T}_{\text {env }}, \quad\left\langle Q_{\text {sup }}(t)\right\rangle \leq k_{\mathrm{B}} \mathrm{T}_{\text {env }},
$$

with $W_{\text {inf }}(t)$ the infimum of the work done on the system and $Q_{\text {sup }}(t)$ the supremum of the heat absorbed by the system in a time $t$. Equation (28) implies that a homogeneous system in isothermal conditions cannot absorb, on average, more than $k_{\mathrm{B}} \mathrm{T}_{\text {env }}$ of energy from the thermal reservoir (regardless of the number of degrees of freedom contained in the system).

\section{PASSAGE PROBABILITIES AND GLOBAL INFIMUM OF ENTROPY PRODUCTION}

Using the theory of martingales, we now derive general expressions for the passage probabilities and the global infimum of entropy production in continuous steady-state processes without jumps.

\section{A. Passage probabilities of entropy production with two asymmetric absorbing boundaries}

We consider the stochastic entropy production $S_{\text {tot }}(\omega ; t)$ of a stationary probability measure $\mathbb{P}$ in a time interval $\left[0, T^{(2)}(\omega)\right]$, which starts at $t=0$ and ends at a stopping time $T^{(2)}(\omega)$. Here, $T^{(2)}$ is the first-passage time at which $S_{\text {tot }}(\omega ; t)$ passes, for the first time, one of the two threshold values $-s_{\text {tot }}^{-}<0$ or $s_{\text {tot }}^{+}>0$ [see Fig. 1(b) for the particular case of $\left.s_{\text {tot }}^{+}=s_{\text {tot }}^{-}\right]$.

We define the passage probability $\mathrm{P}_{+}^{(2)}$ as the probability that entropy production first passes $s_{\text {tot }}^{+}$before passing $-s_{\text {tot }}^{-}$, and, analogously, $\mathrm{P}_{-}^{(2)}$ as the probability that entropy production first passes $-s_{\text {tot }}^{-}$before passing $s_{\text {tot }}^{+}$. These passage probabilities can be written as

$$
\begin{aligned}
& P_{+}^{(2)}=\mathbb{P}\left(\Phi_{+}\right), \\
& \mathbf{P}_{-}^{(2)}=\mathbb{P}\left(\Phi_{-}\right),
\end{aligned}
$$

with $\Phi_{+}$the set of trajectories $\omega$ for which entropy production first passes the positive threshold $s_{\text {tot }}^{+}$, and $\Phi_{-}$ the set of trajectories $\omega$ for which entropy production first passes the negative threshold $-s_{\text {tot }}^{-}$:

$$
\begin{gathered}
\Phi_{+} \equiv\left\{\omega \in \Omega: S_{\mathrm{tot}}\left(\omega ; T^{(2)}(\omega)\right)=s_{\mathrm{tot}}^{+}\right\}, \\
\Phi_{-} \equiv\left\{\omega \in \Omega: S_{\mathrm{tot}}\left(\omega ; T^{(2)}(\omega)\right)=-S_{\mathrm{tot}}^{-}\right\} .
\end{gathered}
$$

Note that if $s_{\text {tot }}^{+}$is different from $s_{\text {tot }}^{-}$, then $\Phi_{+}$and $\Phi_{-}$are not each other's time reversal. Therefore, the probabilities of these sets are, in general, not related by local detailed balance. We also define the conjugate probabilities $\tilde{\mathrm{P}}_{+}^{(2)}$ and $\tilde{P}_{-}^{(2)}$ of the sets $\Phi_{+}$and $\Phi_{-}$under the time-reversed dynamics:

$$
\begin{aligned}
& \tilde{\mathbf{P}}_{+}^{(2)}=(\mathbb{P} \circ \Theta)\left(\Phi_{+}\right), \\
& \tilde{\mathbf{P}}_{-}^{(2)}=(\mathbb{P} \circ \Theta)\left(\Phi_{-}\right) .
\end{aligned}
$$

For a steady-state process out of equilibrium, i.e., $\left\langle S_{\mathrm{tot}}(t)\right\rangle>0, S_{\mathrm{tot}}(t)$ passes one of the two boundaries in a finite time with probability 1 . We thus have

$$
\begin{aligned}
& \mathbf{P}_{+}^{(2)}+\mathbf{P}_{-}^{(2)}=1, \\
& \tilde{\mathbf{P}}_{+}^{(2)}+\tilde{\mathbf{P}}_{-}^{(2)}=1 .
\end{aligned}
$$

In addition, we derive, using Doob's optional sampling theorem, the following two identities:

$$
\begin{aligned}
& \frac{\mathbf{P}_{+}^{(2)}}{\tilde{\mathbf{P}}_{+}^{(2)}}=e^{s_{\mathrm{tot}}^{+} / k_{\mathrm{B}}}, \\
& \frac{\mathbf{P}_{-}^{(2)}}{\tilde{\mathbf{P}}_{-}^{(2)}}=e^{-s_{\mathrm{tot}}^{-} / k_{\mathrm{B}}} .
\end{aligned}
$$

Equation (37) follows from the equalities 


$$
\begin{aligned}
\tilde{\mathbf{P}}_{+}^{(2)} & =\int_{\omega \in \Phi_{+}} \mathrm{d}(\mathbb{P} \circ \Theta) \\
& =\int_{\omega \in \Phi_{+}} e^{-S_{\mathrm{tot}}(\omega ;+\infty) / k_{\mathrm{B}}} \mathrm{d} \mathbb{P} \\
& =\int_{\omega \in \Phi_{+}} e^{-S_{\mathrm{tot}}\left(\omega ; T^{(2)}(\omega)\right) / k_{\mathrm{B}}} \mathrm{d} \mathbb{P} \\
& =e^{-s_{\mathrm{tot}}^{+} / k_{\mathrm{B}}} \int_{\omega \in \Phi_{+}} \mathrm{d} \mathbb{P} \\
& =e^{-s_{\mathrm{tot}}^{+} / k_{\mathrm{B}}} \mathbb{P}\left(\Phi_{+}\right) \\
& =e^{-s_{\mathrm{tot}}^{+} / k_{\mathrm{B}}} \mathbf{P}_{+}^{(2)} .
\end{aligned}
$$

In Eq. (40), we have transformed an integral over the measure $\mathbb{P} \circ \Theta$ to an integral over the measure $\mathbb{P}$, using the definition of entropy production given by Eq. (18) and $e^{-S_{\mathrm{tot}}(\omega ;+\infty) / k_{\mathrm{B}}}=\lim _{t \rightarrow+\infty} e^{-S_{\mathrm{tot}}(\omega ; t) / k_{\mathrm{B}}}$ (see Appendix B). In Eq. (41), we have replaced $e^{-S_{\text {tot }}(\omega ;+\infty) / k_{\mathrm{B}}}$ by its value at the stopping time, $e^{-S_{\text {tot }}\left(\omega ; T^{(2)}(\omega)\right) / k_{\mathrm{B}}}$, using Doob's optional sampling theorem given by Eq. (22). Finally, in Eq. (42), we have used the fact that for continuous processes, $S_{\text {tot }}\left(\omega ; T^{(2)}(\omega)\right)=s_{\text {tot }}^{+}$for all realizations of the process $\omega$ in the set $\Phi_{+}$.

From Eqs. (35)-(38), we find the following explicit expressions for the passage probabilities:

$$
\begin{aligned}
& \mathrm{P}_{+}^{(2)}=\frac{e^{s_{\text {tot }}^{-} / k_{\mathrm{B}}}-1}{e^{s_{\mathrm{tot}}^{-} / k_{\mathrm{B}}}-e^{-s_{\mathrm{tot}}^{+} / k_{\mathrm{B}}}}, \\
& \mathrm{P}_{-}^{(2)}=\frac{1-e^{-s_{\mathrm{tot}}^{+} / k_{\mathrm{B}}}}{e^{s_{\mathrm{tot}}^{-} / k_{\mathrm{B}}}-e^{-s_{\mathrm{tot}}^{+} / k_{\mathrm{B}}}} .
\end{aligned}
$$

For the case of symmetric boundaries $s_{\text {tot }}=s_{\text {tot }}^{+}=s_{\text {tot }}^{-}$, we have

$$
\begin{aligned}
& \mathbf{P}_{+}^{(2)}=\frac{e^{s_{\mathrm{tot}} / k_{\mathrm{B}}}}{1+e^{s_{\mathrm{tot}} / k_{\mathrm{B}}}} \\
& \mathbf{P}_{-}^{(2)}=\frac{1}{1+e^{s_{\mathrm{tot}} / k_{\mathrm{B}}}}
\end{aligned}
$$

We can also discuss the limits where one of the two thresholds becomes large, whereas the other threshold remains finite. This corresponds to a process with one absorbing boundary. If the lower threshold $s_{\text {tot }}^{-} \gg k_{\mathrm{B}}$, the process ends with probability 1 in the positive threshold,

$$
\begin{aligned}
& \mathrm{P}_{+}^{(2)} \simeq 1, \\
& \mathrm{P}_{-}^{(2)} \simeq 0,
\end{aligned}
$$

in accordance with the second law of thermodynamics. If, however, the upper threshold becomes large, $s_{\text {tot }}^{+} \gg k_{\mathrm{B}}$, entropy production can still first reach the positive threshold, but with a probability that depends on $s_{\text {tot }}^{-}$, since on average entropy always increases. In this case, the passage probabilities are given by

$$
\begin{gathered}
\mathrm{P}_{+}^{(2)} \simeq 1-e^{-s_{\mathrm{tot}}^{-} / k_{\mathrm{B}}}, \\
\mathrm{P}_{-}^{(2)} \simeq e^{-s_{\mathrm{tot}}^{-} / k_{\mathrm{B}}}
\end{gathered}
$$

From these limits, we can also determine the passage probabilities $\mathrm{P}_{+}^{(1)}$ and $\mathrm{P}_{-}^{(1)}$ of entropy production with one absorbing boundary. They denote, respectively, the probability to reach a positive boundary $s_{\text {tot }}$ or a negative boundary $-s_{\text {tot }}$ :

$$
\begin{gathered}
\mathbf{P}_{+}^{(1)}=1, \\
\mathbf{P}_{-}^{(1)}=e^{-s_{\mathrm{tot}} / k_{\mathrm{B}}} .
\end{gathered}
$$

The above arguments also hold for sets $\Phi_{+, \mathrm{I}}$ and $\Phi_{-, \mathrm{I}}$ of trajectories $\omega$ that are conditioned on an initial coarsegrained state I. They are defined as the subsets of, respectively, $\Phi_{+}$and $\Phi_{-}$, with the additional constraint that the initial state is part of the coarse-grained state I, i.e., $\omega(0) \in \mathrm{I}$. With these definitions, Eqs. (45) and (46) can be generalized to passage probabilities of entropy production conditioned on the initial state; see Appendix D. Note that this generalization holds for coarse-grained states that are invariant with respect to time reversal, i.e., $\mathrm{I}=\Theta(\mathrm{I})$.

In Fig. 2, we illustrate the expressions of the passage probabilities, given by Eqs. (45) and (46), by plotting $\ln \left(\mathrm{P}_{+}^{(2)} / \mathrm{P}_{-}^{(2)}\right)$ as a function of the thresholds $s_{\text {tot }}^{-}$and $s_{\text {tot }}^{+}$. Characteristic features of this figure are the lines of constant ratio $\mathrm{P}_{+}^{(2)} / \mathrm{P}_{-}^{(2)}$, which are given by

$$
s_{\mathrm{tot}}^{-}=k_{\mathrm{B}} \ln \left(1+\frac{\mathrm{P}_{+}^{(2)}}{\mathrm{P}_{-}^{(2)}}\left(1-e^{-s_{\mathrm{tot}}^{+} / k_{\mathrm{B}}}\right)\right) .
$$

In situations for which $\mathrm{P}_{+}^{(2)}=\mathrm{P}_{-}^{(2)}=1 / 2$, the stopping process is unbiased, and the probability to reach the threshold $s_{\text {tot }}^{+}$equals the probability to reach the threshold $-s_{\text {tot }}^{-}$. Since entropy production is a stochastic process with positive drift, the passage probabilities can be equal only if the negative threshold lies closer to the origin than the positive threshold, $s_{\text {tot }}^{-}<s_{\text {tot }}^{+}$. Additionally, it follows from Eq. (55) that for $\mathrm{P}_{+}^{(2)}=\mathrm{P}_{-}^{(2)}$, the negative threshold obeys $s_{\text {tot }}^{-} \leq k_{\mathrm{B}} \ln 2$, as we illustrate in Fig. 2. This bound on $s_{\text {tot }}^{-}$can also be discussed for passage probabilities $\mathrm{P}_{-}^{(2)} \neq 1 / 2$, for which the lower threshold must satisfy $s_{\text {tot }}^{-} \leq-k_{\mathrm{B}} \ln \mathrm{P}_{-}^{(2)}$.

The discussion of stopping events of entropy production with two boundaries is an example of the thermodynamics of symmetry breaking. Thermodynamics of symmetry 


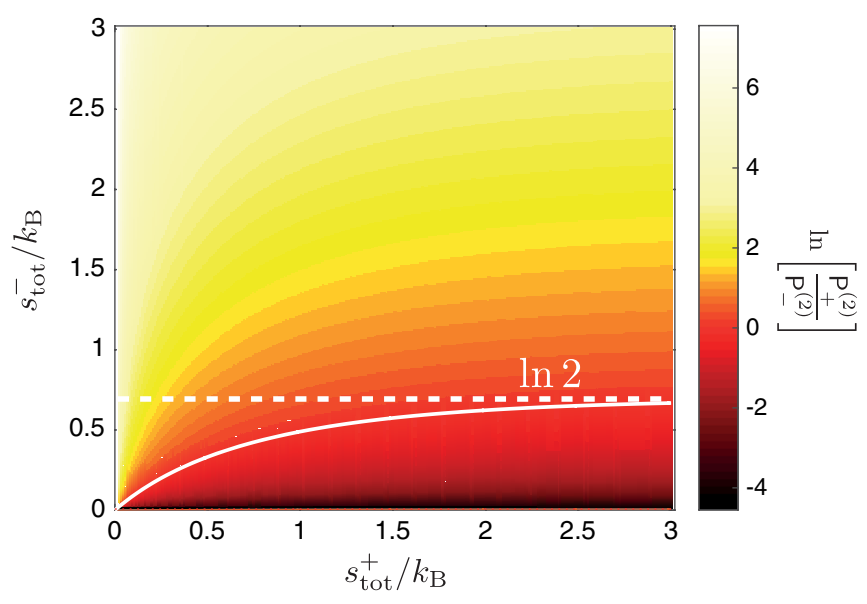

FIG. 2. Entropy-production passage-probability ratio $\ln \left[\mathrm{P}_{+}^{(2)} / \mathrm{P}_{-}^{(2)}\right]$ as a function of the thresholds $s_{\text {tot }}^{-}$and $s_{\text {tot }}^{+}$, obtained from Eqs. (45) and (46). The solid white line is the curve for which $\mathrm{P}_{+}^{(2)}=\mathrm{P}_{-}^{(2)}=1 / 2$, and it follows fromEq. (55). This curve converges asymptotically to the value $s_{\text {tot }}^{-}=k_{\mathrm{B}} \ln 2$ for $s_{\text {tot }}^{+} \rightarrow+\infty$ (white dashed line).

breaking is usually discussed in finite times [62]. Here, we find the analogous relation $\pm s_{\text {tot }}^{ \pm} \geq k_{\mathrm{B}} \ln \mathrm{P}_{ \pm}^{(2)}$, which is valid for stopping times.

\section{B. Global infimum of entropy production}

The global infimum of entropy production $S_{\text {inf }}^{\infty}$ is the lowest value of entropy production over all times $t \geq 0$ during one realization of the process. The global infimum can be defined in terms of the finite-time infima $S_{\text {inf }}(t)$ as

$$
S_{\mathrm{inf}}^{\infty} \equiv \lim _{t \rightarrow \infty} S_{\mathrm{inf}}(t)
$$

Therefore, the global infimum is always negative and smaller than or equal to the finite-time infima $S_{\text {inf }}(t)$. The statistics of global infima follow from the expressions for the passage probabilities (45) and (46). This can be most easily understood in terms of the cumulative distribution of the global infimum

$$
\operatorname{Pr}\left(S_{\text {inf }}^{\infty} \geq-s\right)=\operatorname{Pr}\left(S_{\text {tot }}(t) \geq-s, \forall t \geq 0\right) .
$$

The right-hand side of Eq. (57) is the survival probability for entropy production in a process with one absorbing boundary located at $-s$, with $s \geq 0$. Therefore, the survival probability is the passage probability $\mathrm{P}_{+}^{(2)}$ with $s_{\text {tot }}^{+}=+\infty$ and $s_{\text {tot }}^{-}=s$. This implies $\operatorname{Pr}\left(S_{\text {inf }}^{\infty} \geq-s\right)=1-e^{-s / k_{\mathrm{B}}}$. The corresponding distribution of the global infimum is

$$
p_{S_{\mathrm{inf}}^{\infty}}(-s)=\frac{e^{-s / k_{\mathrm{B}}}}{k_{\mathrm{B}}}
$$

and the mean of the global infimum is

$$
\left\langle S_{\text {inf }}^{\infty}\right\rangle=-k_{\mathrm{B}}
$$

These properties of the global infimum hold for continuous and stationary processes. The infimum law, given by Eq. (26), thus becomes an equality at large times. Since $S_{\text {inf }}(t) \geq S_{\text {inf }}^{\infty}$, the equalities on the global infimum, given by Eqs. (58) and (59), valid for continuous processes, imply the inequalities for the local infima, given by Eqs. (24) and (26), for continuous processes. Note, however, that Eqs. (24) and (26) are also valid for processes in discrete time and processes in continuous time with jumps.

Remarkably, the distribution of the global infimum of entropy production is universal. For any continuous steadystate process, the distribution of the global infimum is an exponential with mean equal to $-k_{\mathrm{B}}$.

\section{STOPPING TIMES OF ENTROPY PRODUCTION}

In this section, we derive fluctuation theorems for stopping times of entropy production using the martingale property of $e^{-S_{\text {tot }}(t) / k_{\mathrm{B}}}$. The stopping-time fluctuation theorem entails fluctuation theorems for first-passage times of entropy production and for waiting times of stochastic processes.

\section{A. Stopping-time fluctuation theorem}

We consider the statistics of $s_{\text {tot }}$-stopping times $T_{+}=$ $T(\omega)$ for which entropy production at the stopping time takes the value $s_{\text {tot }}$, i.e., $S_{\text {tot }}\left(T_{+}\right)=s_{\text {tot }}\left(s_{\text {tot }}>0\right)$. An example of such an $s_{\text {tot }}$-stopping time is the first-passage time $T_{+}^{(1)}$, which determines the time when entropy production $S_{\text {tot }}(t)$ reaches the value $s_{\text {tot }}>0$ for the first time. Another example is given by the first-passage time $T_{+}^{(2)}$, which is the time when entropy production $S_{\text {tot }}(t)$ passes a threshold value $s_{\text {tot }}$ for the first time, given that it has not reached $-s_{\text {tot }}$ before. The latter process is therefore equivalent to a first-passage problem with two absorbing boundaries. More generally, $s_{\text {tot }}$-stopping times $T_{+}^{(n)}$ can be defined by multiple threshold crossings and the condition $S_{\text {tot }}\left(T_{+}^{(n)}\right)=s_{\text {tot }}$, with $n$ the order of threshold crossings.

We derive the following cumulative fluctuation theorem for $s_{\text {tot }}$-stopping times $T_{+}$(see Appendix E):

$$
\frac{\mathbb{P}\left(\Phi_{T_{+} \leq t}\right)}{\mathbb{P}\left(\Theta_{T_{+}}\left(\Phi_{T_{+} \leq t}\right)\right)}=e^{s_{\text {tot }} / k_{\mathrm{B}}},
$$

where $\mathbb{P}\left(\Phi_{T_{+} \leq t}\right)$ is the probability to observe a trajectory $\omega$ that satisfies the stopping-time criterion at a time $T_{+} \leq t<+\infty$, and $\Phi_{T_{+} \leq t}$ denotes the set of these trajectories. The set $\Theta_{T_{+}}\left(\Phi_{T_{+} \leq t}\right)$ describes the time-reversed trajectories of $\Phi_{T_{+} \leq t}$. It is generated by applying the timereversal map $\Theta_{T_{+}}$to all the elements of the original set. The 
map $\Theta_{T_{+}}=T_{T_{+}} \circ \Theta$ time reverses trajectories $\omega$ with respect to the reference time $T_{+}(\omega) / 2$, and thus $X\left(\Theta_{T_{+}}(\omega) ; \tau\right)=X\left(\omega ; T_{+}(\omega)-\tau\right)$, for stochastic processes $X$ that are even under time reversal. The fluctuation theorem for $s_{\text {tot }}$-stopping times, Eq. (60), is valid for continuous and stationary stochastic processes.

The probability density $p_{T_{+}}$of the $s_{\text {tot }}$-stopping time $T_{+}$ is given by

$$
p_{T_{+}}\left(t ; s_{\mathrm{tot}}\right)=\frac{\mathrm{d}}{\mathrm{d} t} \mathbb{P}\left(\Phi_{T_{+} \leq t}\right),
$$

for $t<+\infty$. Entropy production is odd under time reversal, i.e., $S_{\text {tot }}\left(\Theta_{T_{+}}(\omega) ; T_{+}(\omega)\right)=-S_{\text {tot }}\left(\omega ; T_{+}(\omega)\right)$, as shown in Appendix B 1. Therefore, we can associate with the $s_{\text {tot }}$-stopping time $T_{+}$a $\left(-s_{\text {tot }}\right)$-stopping time $T_{-}$with the property

\section{(a) One absorbing boundary}
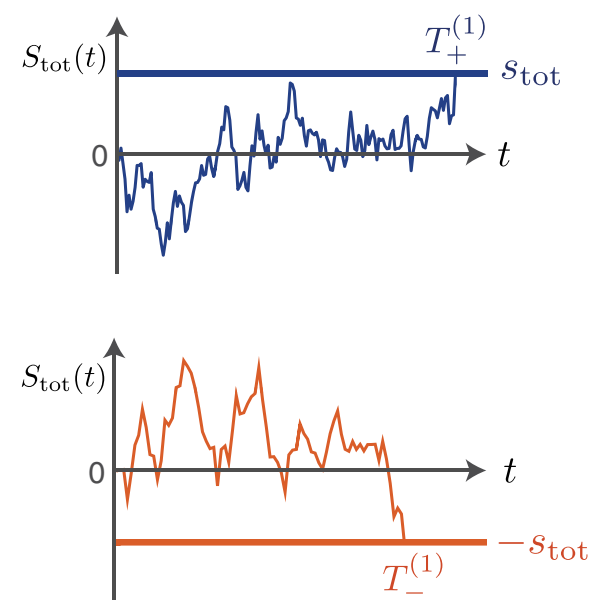

(b) Two absorbing boundaries

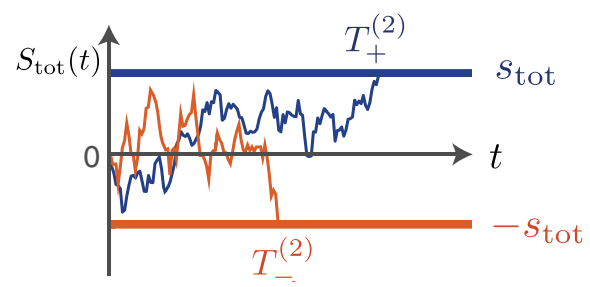

FIG. 3. Illustration of the first-passage times for entropy production. (a) First-passage time $T_{+}^{(1)}$ for entropy production with one positive absorbing boundary $s_{\text {tot }}$ (top) and first-passage time $T_{-}^{(1)}$ for entropy production with one negative absorbing boundary $-s_{\text {tot }}$ (bottom). (b) First-passage times $T_{+}^{(2)}$ and $T_{-}^{(2)}$ for entropy production with two absorbing boundaries at $\pm s_{\text {tot }}$. At the time $T_{+}^{(2)}$, entropy production passes the threshold $s_{\text {tot }}$ for the first time without having reached $-s_{\text {tot }}$ before. At the time $T_{-}^{(2)}$, entropy production passes the threshold $-s_{\text {tot }}$ for the first time without having reached $s_{\text {tot }}$ before.

$$
\Theta_{T_{+}}\left(\Phi_{T_{+} \leq t}\right)=\Phi_{T_{-} \leq t} .
$$

For example, the $\left(-s_{\text {tot }}\right)$-stopping time $T_{-}$associated with the first-passage time $T_{+}^{(1)}$ is the first-passage time $T_{-}^{(1)}$ when entropy production first reaches $-s_{\text {tot }}$; see Fig. 3(a). Analogously, the $\left(-S_{\text {tot }}\right)$-stopping time $T_{-}$associated with the first-passage time $T_{+}^{(2)}$ is the first-passage time $T_{-}^{(2)}$ when entropy production first reaches $-s_{\text {tot }}$ without having reached $s_{\text {tot }}$ before; see Fig. 3(b).

We can thus identify the distribution of $T_{-}$with the measure of time-reversed trajectories:

$$
p_{T_{-}}\left(t ;-s_{\mathrm{tot}}\right)=\frac{\mathrm{d}}{\mathrm{d} t} \mathbb{P}\left(\Theta_{T_{+}}\left(\Phi_{T_{+} \leq t}\right)\right) .
$$

This equation can be applied to all pairs of stopping times $T_{+}$and $T_{-}$related by Eq. (62). From Eqs. (60), (61), and (63), we get the stopping-time fluctuation theorem for entropy production,

$$
\frac{p_{T_{+}}\left(t ; s_{\mathrm{tot}}\right)}{p_{T_{-}}\left(t ;-s_{\mathrm{tot}}\right)}=e^{s_{\mathrm{tot}} / k_{\mathrm{B}}}
$$

The stopping-time fluctuation theorem for entropy production, given by Eq. (64), generalizes the results derived in Ref. [47] for first-passage times. Below, we discuss two interesting implications of Eq. (64) for the stopping-time statistics of entropy production.

\section{B. Symmetry of the normalized stopping-time distributions}

The stopping-time fluctuation relation Eq. (64) implies an equality between the normalized stopping-time distributions $p_{T_{+}}\left(t \mid s_{\text {tot }}\right)$ and $p_{T_{-}}\left(t \mid-s_{\text {tot }}\right)$, which reads

$$
p_{T_{+}}\left(t \mid s_{\text {tot }}\right)=p_{T_{-}}\left(t \mid-s_{\text {tot }}\right) .
$$

The normalized distributions are defined as

$$
\begin{gathered}
p_{T_{+}}\left(t \mid s_{\mathrm{tot}}\right) \equiv \frac{p_{T_{+}}\left(t ; s_{\mathrm{tot}}\right)}{\int_{0}^{\infty} \mathrm{d} t p_{T_{+}}\left(t ; s_{\mathrm{tot}}\right)}, \\
p_{T_{-}}\left(t \mid-s_{\mathrm{tot}}\right) \equiv \frac{p_{T_{-}}\left(t ;-s_{\mathrm{tot}}\right)}{\int_{0}^{\infty} \mathrm{d} t p_{T_{-}}\left(t ;-s_{\mathrm{tot}}\right)} .
\end{gathered}
$$

The symmetric relation Eq. (65) comes from the fact that the ratio of the stopping-time distributions in Eq. (64) is time independent. Consequently, the stopping-time statistics for entropy production are identical for the ensembles with stopping events for positive and negative entropy production.

The stopping-time fluctuation theorem (64) thus implies that the mean stopping time, given that the process terminates at the positive boundary, is equal to the mean 
stopping time, given that the process terminates at the negative boundary:

$$
\left\langle T_{+}\right\rangle=\left\langle T_{-}\right\rangle,
$$

with $\quad\left\langle T_{+}\right\rangle=\int_{0}^{+\infty} \mathrm{d} t t p_{T_{+}}\left(t \mid s_{\text {tot }}\right) \quad$ and $\quad\left\langle T_{-}\right\rangle=$ $\int_{0}^{+\infty} \mathrm{d} t t p_{T_{-}}\left(t \mid-s_{\text {tot }}\right)$. This remarkable symmetry extends to all the moments of the stopping-time distributions. A similar result has been found for waiting-time distributions in chemical kinetics [63-69], for cycle time distributions in Markov chains [68,70], and for decision-time distributions in sequential hypothesis tests $[47,71]$. These results could therefore be interpreted as a consequence of the fundamental relation Eq. (65) for stopping-time fluctuations of entropy production.

\section{Passage probabilities for symmetric boundaries}

Equation (64) implies the following relation for the ratio of the passage probabilities of entropy production:

$$
\frac{\mathrm{P}_{+}}{\mathrm{P}_{-}}=e^{s_{\mathrm{tot}} / k_{\mathrm{B}}}
$$

Passage probabilities are the probabilities that entropy production satisfies the stopping-time criterion in a finite time. They read

$$
\begin{aligned}
& \mathrm{P}_{+}=\int_{0}^{\infty} \mathrm{d} t p_{T_{+}}\left(t ; s_{\mathrm{tot}}\right), \\
& \mathrm{P}_{-}=\int_{0}^{\infty} \mathrm{d} t p_{T_{-}}\left(t ;-s_{\mathrm{tot}}\right) .
\end{aligned}
$$

Equation (69) follows directly from integrating Eq. (64) over time. The relations (53) and (54) for passage probabilities of entropy production with one absorbing boundary, and the relations (47) and (48) for passage probabilities of entropy production with two symmetric absorbing boundaries, are examples of passage probabilities that satisfy Eq. (69).

\section{Fluctuation relation for waiting times}

An interesting question, closely related to entropy stopping times, is the following: What is the waiting time $T^{\mathrm{I} \rightarrow \mathrm{II}}$ a process takes to travel from state I to state II. Here, we derive exact relations characterizing $\left( \pm s_{\text {tot }}\right)$-waiting times $T_{ \pm}^{\mathrm{I} \rightarrow \mathrm{II}}$. The $\left( \pm s_{\text {tot }}\right)$-waiting time $T_{ \pm}^{\mathrm{I} \rightarrow \mathrm{II}}$ denotes the time a process takes to travel from state I to state II and to produce a total positive or negative entropy $\pm s_{\text {tot }}$ (see Appendix E 4).

Following Kramers [72], we define states as points in phase space, i.e., I $=\left\{\boldsymbol{q}_{\mathrm{I}}\right\}$ and II $=\left\{\boldsymbol{q}_{\mathrm{II}}\right\}$. In Appendix E 4, we also consider the more general case for which states consist of sets of points, which may also contain odd-parity variables.

We first derive a cumulative fluctuation theorem that applies to trajectories starting from a given initial state I (see Appendix E 3):

$$
\frac{\mathbb{P}\left(\Phi_{T_{+} \leq t} \cap \Gamma_{\mathrm{I}}\right)}{\mathbb{P}\left(\Theta_{T_{+}}\left(\Phi_{T_{+} \leq t} \cap \Gamma_{\mathrm{I}}\right)\right)}=e^{s_{\mathrm{tot}} / k_{\mathrm{B}}}
$$

with $\Gamma_{\text {I }}$ the set of trajectories $\omega$ for which $\omega(0) \in \mathrm{I}$.

We use Eq. (72) to derive the following fluctuation theorem for waiting times (see Appendix E 4):

$$
\frac{p_{T_{+}^{\mathrm{I} \rightarrow \mathrm{II}}}(t)}{p_{T_{-}^{\mathrm{II}} \rightarrow I}(t)}=e^{s_{\mathrm{env}} / k_{\mathrm{B}}},
$$

where $s_{\text {env }}=s_{\text {tot }}+k_{\mathrm{B}} \log \frac{p_{\mathrm{ss}}\left(\boldsymbol{q}_{\mathrm{II}}\right)}{p_{\mathrm{ss}}\left(\boldsymbol{q}_{\mathrm{I}}\right)}$ is the change in the environment entropy during the transition from state I to state II. Equation (73) relates the waiting-time distributions between two states with the environment-entropy change along trajectories connecting both states.

We normalize the distributions in Eq. (73) and find a relation for the normalized waiting-time distributions,

$$
p_{T_{+}^{\mathrm{I} \rightarrow \mathrm{II}}}\left(t \mid s_{\mathrm{env}}\right)=p_{T_{-}^{\mathrm{II} \rightarrow \mathrm{I}}}\left(t \mid-s_{\mathrm{env}}\right),
$$

and for the associated passage probabilities,

$$
\frac{\mathrm{P}_{+}^{\mathrm{I} \rightarrow \mathrm{II}}}{\mathrm{P}_{-}^{\mathrm{II} \rightarrow \mathrm{I}}}=e^{s_{\mathrm{env}} / k_{\mathrm{B}}} .
$$

Interestingly, the relations Eqs. (73)-(75) are similar to the stopping-time relations (64), (65), and (69) discussed above. However, in Eqs. (73)-(75), the environmental entropy production appears, instead of the total entropy production, because the trajectories are conditioned on passage through initial and final states. For isothermal processes, $s_{\text {env }}=-Q / \mathrm{T}_{\text {env }}$, with $Q$ the heat absorbed by the system and $\mathrm{T}_{\text {env }}$ the temperature of the environment.

\section{APPLICATION TO SIMPLE COLLOIDAL SYSTEMS}

Infima of entropy-production fluctuations, and stopping times of entropy production, can be calculated for specific stochastic processes. In this section, we discuss these quantities for the dynamics of a colloidal particle with diffusion coefficient $D$, which moves in a periodic potential $V$ with period $\ell$, and under the influence of a constant external force $F$ [73,74] (see Fig. 4 for a graphical illustration). This process has been realized in several experiments using colloidal particles trapped with toroidal optical potentials [30,75-77]. We discuss how our results can be tested in these types of experiments.

We describe the dynamics of this colloidal particle in terms of a one-dimensional overdamped Brownian motion with periodic boundary conditions. The state of the particle at time $t$ is characterized by a phase variable $\phi(t) \in[0, \ell)$. In the illustration of a ring geometry in Fig. $4, \phi$ is the azimuthal angle and $\ell=2 \pi$. Equivalently, one can consider a stochastic process $X(t)$ given by the net distance 


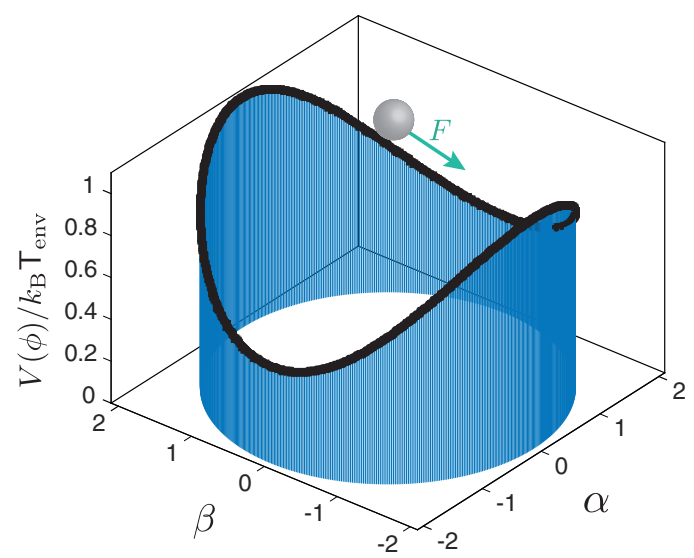

FIG. 4. Illustration of a Smoluchowski-Feynman ratchet. A Brownian particle (gray sphere) immersed in a thermal bath of temperature $\mathrm{T}_{\text {env }}$ moves in a periodic potential $V(\phi)$ (black shaded curve) with friction coefficient $\gamma$. The coordinate $\phi$ is the azimuthal angle of the particle. When applying an external force $F=\gamma v$ in the azimuthal direction, the particle reaches a nonequilibrium steady state. In this example, $V(\phi)=$ $k_{\mathrm{B}} \mathrm{T}_{\text {env }} \ln [\cos (\phi)+2], \quad \alpha=R \cos (\phi)$ and $\beta=R \sin (\phi)$, with $R=2$.

traveled by the Brownian particle up to time $t$ : $X(t)=\phi(t)-\ell N(t)$, where $N(t)=N_{-}(t)-N_{+}(t)$ is the negative winding number, or the number of turns $N_{-}(t)$ against the direction of the external force minus the number of turns $N_{+}(t)$ in the direction of the external force, of the particle up to time $t$ [78]. The time evolution of $X(t)$ obeys the Langevin equation

$$
\frac{\mathrm{d} X(t)}{\mathrm{d} t}=-\frac{1}{\gamma} \frac{\partial V(X(t))}{\partial x}+v+\zeta(t)
$$

where $\gamma$ is the friction coefficient, $v=F / \gamma$ is the drift velocity, and $\zeta$ is a Gaussian white noise with zero mean $\langle\zeta(t)\rangle=0 \quad$ and $\quad$ with autocorrelation $\left\langle\zeta(t) \zeta\left(t^{\prime}\right)\right\rangle=$ $2 D \delta\left(t-t^{\prime}\right)$. If the Einstein relation holds, $D=k_{\mathrm{B}} \mathrm{T}_{\text {env }} / \gamma$, with $\mathrm{T}_{\text {env }}$ the temperature of the thermal reservoir. In Eq. (76), $V(x)$ is the periodic potential of period $\ell$, $V(x+\ell)=V(x)$, and $\frac{\partial V(X(t))}{\partial x}=\left.\frac{\partial V(x)}{\partial x}\right|_{X(t)}$.

The steady-state entropy production after a time $t$ is $[73,79]$

$$
S_{\mathrm{tot}}(t)=k_{\mathrm{B}} \ln \frac{\int_{X(0)+\ell}^{X(0)+\ell} \mathrm{d} y e^{(V(y)-F y) / k_{\mathrm{B}} \mathrm{T}_{\mathrm{env}}}}{\int_{X(t)}^{X(t)+\ell} \mathrm{d} y e^{(V(y)-F y) / k_{\mathrm{B}} \mathrm{T}_{\mathrm{env}}}} .
$$

For the drift-diffusion process with $V(x)=0$, the total entropy production reads

$$
S_{\mathrm{tot}}(t)=k_{\mathrm{B}} \frac{v}{D}[X(t)-X(0)] .
$$

Equation (78) implies that the first-passage and extremevalue statistics of entropy production in the drift-diffusion process follow from the statistics of the position $X(t)$ of a drifted Brownian particle on the real line. Consequently, the drift-diffusion process is an example for which the infimum and first-passage statistics of entropy production can be calculated analytically. We also consider a SmoluchowskiFeynman ratchet whose dynamics is given by Eq. (76) with the nonzero potential

$$
V(x)=V_{0} \ln (\cos (2 \pi x / \ell)+2),
$$

as illustrated in Fig. 4. For the potential (79) with $V_{0}=k_{\mathrm{B}} \mathrm{T}_{\text {env }}$, the stochastic entropy production in a steady state, given by Eq. (77), equals [79]

$$
\frac{S_{\mathrm{tot}}(t)}{k_{\mathrm{B}}}=\frac{F}{k_{\mathrm{B}} \mathrm{T}_{\mathrm{env}}}(X(t)-X(0))-\ln \frac{\psi(X(t), f)}{\psi(X(0), f)},
$$

with $f=F \ell /\left(2 \pi k_{\mathrm{B}} \mathrm{T}_{\text {env }}\right)$ and $\psi(x, f)=f^{2}[\cos (2 \pi x / \ell)+$ 2] $-f \sin (2 \pi x / \ell)+2$.

In the following, we present analytical results for the infima and passage statistics of entropy production in the drift-diffusion process, and we present simulation results for the Smoluchowski-Feynman ratchet with the potential (79). The simulation results are for parameters corresponding to conditions in optical tweezers experiments [75-77], namely, for a polystyrene spherical Brownian particle of radius $1 \mu \mathrm{m}$ immersed in water at room temperature.

\section{A. Infimum statistics}

We present the statistics of infima of entropy production for the drift-diffusion process and the SmoluchowskiFeynman ratchet in a steady state.

In Fig. 5, we show that the cumulative distribution of the finite-time entropy-production infima $S_{\text {inf }}(t)$ is bounded from below by $1-e^{-s}$, which confirms the universality of Eq. (2). We compare analytical results for the driftdiffusion process $[V(x)=0$, dashed lines] with numerical results for the Smoluchowski-Feynman ratchet [with a potential $V(x)$ given by Eq. (79), solid lines], for different values of the mean entropy production $\bar{s}(t)=\left\langle S_{\text {tot }}(t)\right\rangle / k_{\mathrm{B}}$. The analytical expression for the cumulative distribution of $S_{\text {inf }}(t)$ is, for the drift-diffusion process, given by (see Appendix F)

$$
\begin{aligned}
\operatorname{Pr}\left(\frac{S_{\text {inf }}(t)}{k_{\mathrm{B}}} \geq-s\right)= & \frac{1}{2}\left[\operatorname{erfc}\left(\frac{-s-\bar{s}(t)}{2 \sqrt{\bar{s}(t)}}\right)\right. \\
& \left.-e^{-s} \operatorname{erfc}\left(\frac{s-\bar{s}(t)}{2 \sqrt{\bar{s}(t)}}\right)\right],
\end{aligned}
$$

where $s>0$, $\operatorname{erfc}(x)=(2 / \sqrt{\pi}) \int_{x}^{+\infty} e^{-y^{2}} \mathrm{~d} y$ is the complementary error function, and $\bar{s}(t)$ is the average entropy production in a steady state at time $t$, which for the driftdiffusion process is $\bar{s}(t)=\left(v^{2} / D\right) t$. Interestingly, the bound saturates for large values of the average entropy 


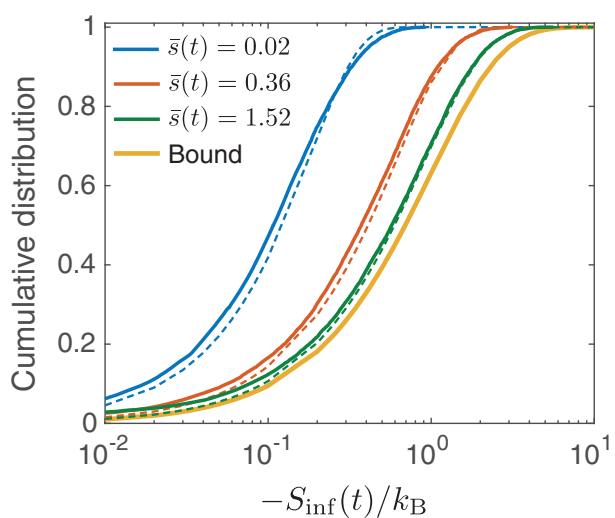

FIG. 5. Cumulative distributions of the infimum of entropy production for a Smoluchowski-Feynman ratchet in a steady state for different values of the mean entropy change $\bar{s}(t)=\left\langle S_{\text {tot }}(t)\right\rangle / k_{\mathrm{B}}$. Simulation results for the SmoluchowskiFeynman ratchet with the potential (79) (solid lines) are compared with analytical results for the drift-diffusion process given by Eq. (81) (dashed lines), and with the universal bound given by $1-e^{-s}$ (rightmost yellow curve). Simulation parameters: $10^{4}$ simulations with $\mathrm{T}_{\text {env }}=300 \mathrm{~K}, \ell=2 \pi \mathrm{nm}, \gamma=8.4 \mathrm{pN} \mathrm{s} / \mathrm{nm}$, $V_{0}=k_{\mathrm{B}} \mathrm{T}_{\text {env }}$, simulation time step $\Delta t=0.00127 \mathrm{~s}$, and total simulation time $t=0.4 \mathrm{~s}$. The external force $F$ and the drift velocity $v$ are determined by the average entropy production $\bar{s}(t)$.

production $\bar{s}(t)$, which illustrates the universal equality in the distribution of the global infimum of entropy production, given by Eq. (3). Remarkably, as shown in Fig. 5, the cumulative distribution for the infimum of entropy production of the Smoluchowski-Feynman ratchet is nearly identical for different shapes of the potential $V(x)$. This equivalence between the infimum cumulative distributions holds to a good approximation also for small values of $\bar{s}(t)$, where the shape of the potential $V(x)$ affects the entropyproduction fluctuations.

Second, in Fig. 6, we illustrate the infimum law, $\left\langle S_{\text {inf }}(t)\right\rangle \geq-k_{\mathrm{B}}$. We show the average finite-time infimum $\left\langle S_{\text {inf }}(t)\right\rangle$ as a function of the mean entropy production $\bar{s}(t)$; we compare analytical results for the drift-diffusion process (dashed lines) with numerical results for the Smoluchowski-Feynman ratchet with a potential given by Eq. (79) (solid lines). The analytical expression for the drift-diffusion process is (see Appendix F)

$$
\begin{aligned}
\left\langle\frac{S_{\mathrm{inf}}(t)}{k_{\mathrm{B}}}\right\rangle= & -\operatorname{erf}\left(\frac{\sqrt{\bar{s}(t)}}{2}\right)+\frac{\bar{s}(t)}{2} \operatorname{erfc}\left(\frac{\sqrt{\bar{s}(t)}}{2}\right) \\
& -\sqrt{\frac{\bar{s}(t)}{\pi}} e^{-\bar{s}(t) / 4},
\end{aligned}
$$

where $\operatorname{erf}(x)=1-\operatorname{erfc}(x)$ is the error function. In the limit of large times, the global infimum $\left\langle S_{\text {inf }}^{\infty}\right\rangle=-k_{\mathrm{B}}$, in accordance with the universal equality (4). The results in Fig. 6 show that the mean finite-time infimum of entropy production has a functional dependence on $\bar{s}(t)$ that is to a

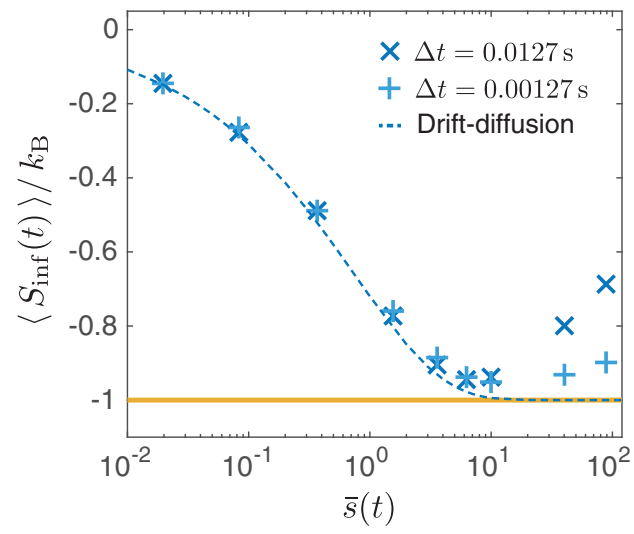

FIG. 6. Mean of the finite-time entropy-production infimum $\left\langle S_{\text {inf }}(t)\right\rangle$ as a function of the mean entropy change $\bar{s}(t)=$ $\left\langle S_{\text {tot }}(t)\right\rangle / k_{\mathrm{B}}$ for a Smoluchowski-Feynman ratchet in a steady state. Simulation results for the Smoluchowski-Feynman ratchet with the potential (79) (symbols) are compared with analytical results for the drift-diffusion process given by Eq. (82) (dashed lines), and with the universal infimum law given by $-k_{\mathrm{B}}$ (yellow thick bar). Different symbols are obtained for different simulation time steps $\Delta t$. Simulation parameters: $10^{4}$ simulations with $\mathrm{T}_{\text {env }}=300 \mathrm{~K}, \quad \ell=2 \pi \mathrm{nm}, \quad \gamma=8.4 \mathrm{pN} \mathrm{s} / \mathrm{nm}, \quad V_{0}=k_{\mathrm{B}} \mathrm{T}_{\text {env }}$, and total simulation time $t=0.4 \mathrm{~s}$.

good approximation independent of the potential $V(x)$. This points towards a universal behavior of the statistics of local infima of entropy production.

\section{B. Passage probabilities and first-passage times}

\section{Symmetric boundaries}

We illustrate our universal results on passage probabilities and first-passage times for the drift-diffusion process and the Smoluchowski-Feynman ratchet.

For the drift-diffusion process, we recover the firstpassage-time fluctuation theorem for entropy production, given by Eq. (8), from the analytical expressions of the first-passage-time distributions for the position of a Brownian particle (see Appendix G).

We also compute, using numerical simulations, the firstpassage-time statistics for entropy production for the Smoluchowski-Feynman ratchet in a steady state and with potential $V(x)$ given by Eq. (79). First, we study the firstpassage times $T_{ \pm}^{(2)}$ for entropy production with two absorbing boundaries at the threshold values $s_{\text {tot }}$ and $-s_{\text {tot }}$ (with $s_{\text {tot }}>0$ ). Figure 7 shows the empirical firstpassage-time distribution $p_{T_{+}^{(2)}}\left(t ; s_{\text {tot }}\right)$ to first reach the positive threshold (blue squares) together with the firstpassage-time distribution $p_{T_{-}^{(2)}}\left(t ;-s_{\text {tot }}\right)$ to first reach the negative threshold (red circles; the distribution is rescaled by $\left.e^{s_{\text {tot }} / k_{\mathrm{B}}}\right)$. Since both distributions coincide, we confirm the validity of the first-passage-time fluctuation theorem given by Eq. (8). Moreover, the functional dependence of the empirical passage probabilities $\mathrm{P}_{+}^{(2)}$ and $\mathrm{P}_{-}^{(2)}$ on the 


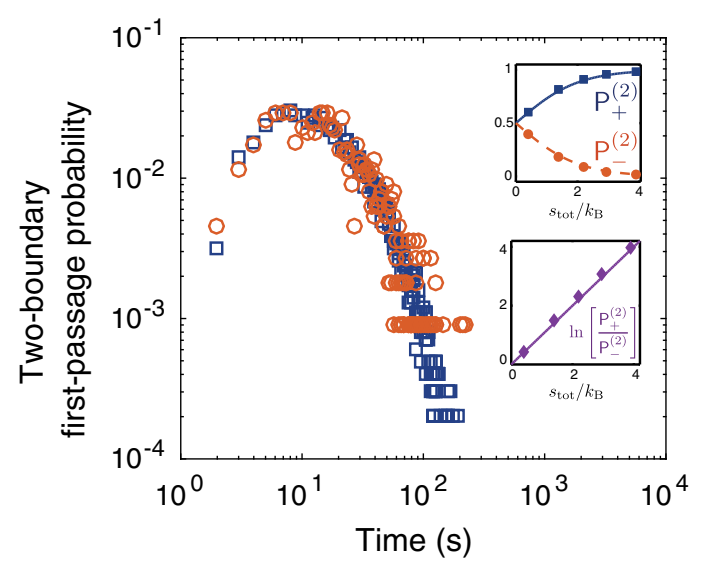

FIG. 7. Empirical two-boundary first-passage-time distributions of entropy production to first reach a positive threshold $p_{T_{+}^{(2)}}\left(t ; s_{\text {tot }}\right)$ (blue squares) and the rescaled distribution for the negative threshold $p_{T_{-}^{(2)}}\left(t ;-s_{\text {tot }}\right) e^{s_{\text {tot }} / k_{\mathrm{B}}}$ (red circles) for the Smoluchowski-Feynman ratchet with the potential (79) in a steady state. The distributions are obtained from $10^{4}$ numerical simulations, and the threshold values are set to $\pm s_{\text {tot }}= \pm 2.2 k_{\mathrm{B}}$. The simulations are done using the Euler numerical scheme with the following parameters: $F=0.64 \mathrm{pN}, \quad \mathrm{T}_{\text {env }}=300 \mathrm{~K}$, $\ell=2 \pi \mathrm{nm}, \gamma=8.4 \mathrm{pN} \mathrm{s} / \mathrm{nm}, V_{0}=k_{\mathrm{B}} \mathrm{T}_{\text {env }}$, and simulation time step $\Delta t=0.0127 \mathrm{~s}$. Top inset: Empirical passage probabilities of entropy production to first reach the positive threshold $\left(\mathrm{P}_{+}^{(2)}\right.$, blue squares) and to first reach the negative threshold $\left(\mathrm{P}_{-}^{(2)}\right.$, red circles) as a function of the threshold value $s_{\text {tot }}$. The analytical expressions for $\mathrm{P}_{+}^{(2)}$ (blue solid line), given by Eq. (47), and for $\mathrm{P}_{-}^{(2)}$ (red dashed line), given by Eq. (48), are also shown. Bottom inset: Logarithm of the ratio between the empirical passage probabilities $\mathrm{P}_{+}^{(2)}$ and $\mathrm{P}_{-}^{(2)}$ as a function of the threshold value $s_{\text {tot }}$ (magenta diamonds). The solid line is a straight line of slope 1.

threshold value $s_{\text {tot }}$ obeys the expressions given by Eqs. (47) and (48) (see top inset in Fig. 7). As a result, the integral first-passage-time fluctuation theorem given by Eq. (69) is also fulfilled in this example (see bottom inset in Fig. 7). Notice that, for this example, analytical expressions for $\mathrm{P}_{+}^{(2)}$ and $\mathrm{P}_{-}^{(2)}$ can be obtained with the method described in Refs. [80,81].

As a second case, we consider two one-boundary firstpassage problems for entropy production. We study the first-passage times $T_{+}^{(1)}$ for entropy production with one absorbing boundary at the threshold value $s_{\text {tot }}$, and the corresponding first-passage times $T_{-}^{(1)}$ at the negative threshold value $-s_{\text {tot }}$. We obtain numerical estimates of the distribution $p_{T_{+}^{(1)}}\left(t ; s_{\text {tot }}\right)$ and its conjugate distribution $p_{T_{-}^{(1)}}\left(t ;-s_{\text {tot }}\right)$. Figure 8 shows empirical estimates of these first-passage-time distributions and confirms the validity of the first-passage-time fluctuation theorem given by Eq. (8). In the top inset of Fig. 8, we show that the passage probabilities $\mathrm{P}_{+}^{(1)}$ and $\mathrm{P}_{-}^{(1)}$ are given by the analytical expressions in Eqs. (53) and (54), and in the bottom inset, we verify the integral first-passage-time fluctuation

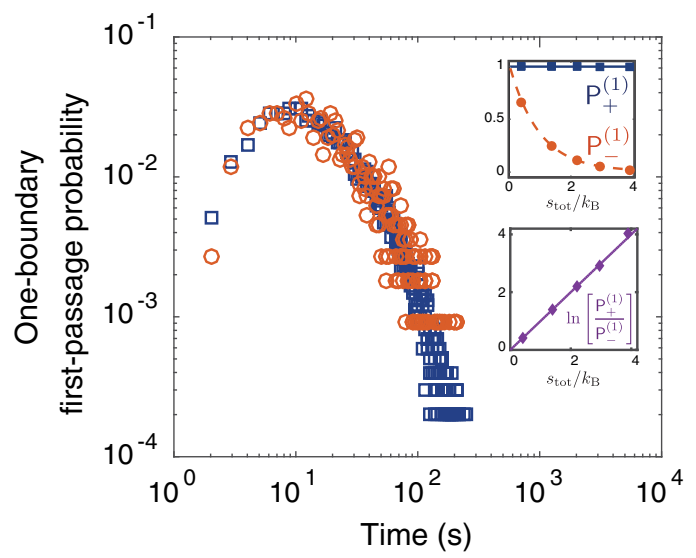

FIG. 8. Empirical one-boundary first-passage-time distributions of entropy production to first reach a positive threshold $p_{T_{+}^{(1)}}\left(t ; s_{\text {tot }}\right)$ (blue squares), and the rescaled distribution for the negative threshold $p_{T_{-}^{(1)}}\left(t ;-s_{\text {tot }}\right) e^{s_{\mathrm{tot}} / k_{\mathrm{B}}}$ (red circles), for the Smoluchowski-Feynman ratchet with the potential (79) in a steady state. The estimate of $p_{T_{+}^{(1)}}\left(t ; s_{\text {tot }}\right)\left[p_{T_{-}^{(1)}}\left(t ;-s_{\text {tot }}\right)\right]$ is obtained by measuring the time when entropy production first reaches the single absorbing boundary $s_{\text {tot }}=2.2 k_{\mathrm{B}}$ $\left(-s_{\text {tot }}=-2.2 k_{\mathrm{B}}\right)$ in $10^{4}$ simulations. The simulations are done with the same parameters as in Fig. 7, and the empirical probabilities are calculated over a total simulation time of $\tau_{\max }=20$ s. Top inset: Empirical passage probabilities of entropy production with a positive-threshold $\left(\mathrm{P}_{+}^{(1)}\right.$, blue squares) and with a negative-threshold $\left(\mathrm{P}_{-}^{(1)}\right.$, red circles) as a function of the values of the thresholds. For comparison, the expression for $\mathbf{P}_{+}^{(1)}$ given by Eq. (53) (blue solid line) and the expression for $\mathbf{P}_{-}^{(1)}$ given by Eq. (54) (red dashed line) are also shown. Bottom inset: Logarithm of the ratio between $\mathrm{P}_{+}^{(1)}$ and $\mathrm{P}_{-}^{(1)}$ as a function of the threshold value (magenta diamonds). The solid line is a straight line of slope 1 .

theorem given by Eq. (69) for one-boundary first-passage processes.

\section{Asymmetric boundaries}

We now discuss the passage statistics for entropy production with asymmetric boundaries. In Appendix G, we discuss the drift-diffusion process, whereas here we discuss the Smoluchowski-Feynman rachet with a potential given by Eq. (79). In Fig. 9(a), we show the distributions of the first-passage times $p_{T_{+}^{(2)}}\left(t ; s_{\text {tot }}^{+}\right)$and $p_{T_{-}^{(2)}}\left(t ;-s_{\text {tot }}^{-}\right)$with two boundaries located at $s_{\text {tot }}^{+}=2 k_{\mathrm{B}}$ and $-s_{\text {tot }}^{-}=-k_{\mathrm{B}}$. Interestingly, the ratio of the two distributions $p_{T_{+}^{(2)}}\left(t ; s_{\text {tot }}^{+}\right) / p_{T_{-}^{(2)}}\left(t ;-s_{\text {tot }}^{-}\right)$is time dependent. Therefore, the first-passage-time fluctuation relation, given by Eq. (8), does not extend to asymmetric boundaries. Consequently, for asymmetric boundaries, the mean firstpassage times $\left\langle T_{+}^{(2)}\right\rangle$ and $\left\langle T_{-}^{(2)}\right\rangle$ to first reach, respectively, the positive and negative boundaries are, in general, different. In Fig. 9(b), we show numerical results for the 

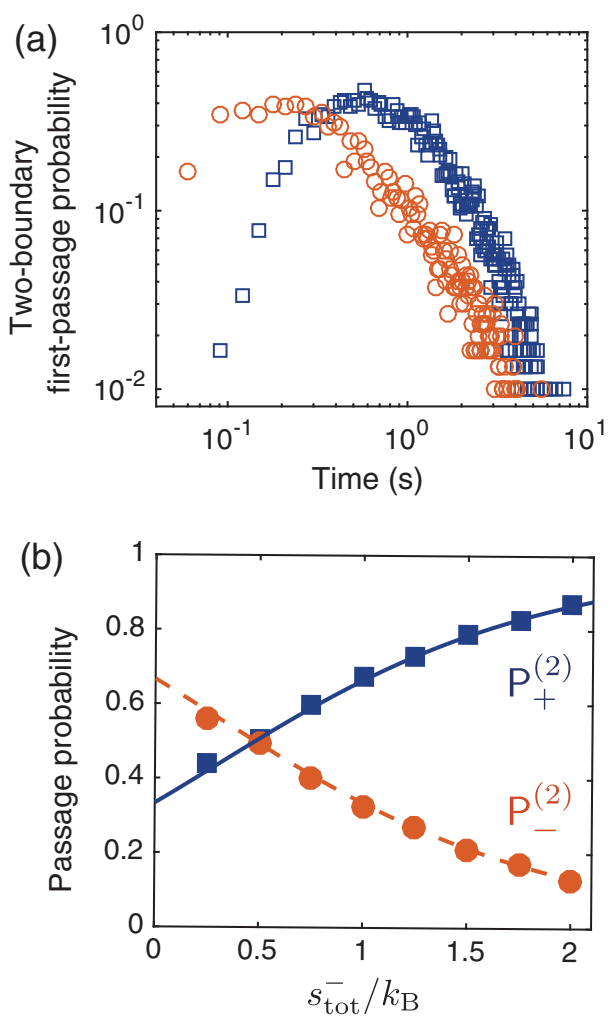

FIG. 9. First-passage statistics of entropy production with two absorbing and asymmetric boundaries at values $s_{\text {tot }}^{+}=2 s_{\text {tot }}^{-}$for the Smoluchowski-Feynman ratchet with potential (79) in a steady state. (a) The first-passage-time distribution $p_{T_{+}^{(2)}}\left(t ; s_{\text {tot }}^{+}\right)$(blue open squares) for entropy production to first reach $s_{\text {tot }}^{+}=2 k_{\mathrm{B}}$ given that it has not reached $-s_{\text {tot }}^{-}=-k_{\mathrm{B}}$ before, and the firstpassage-time distribution $p_{T_{-}^{(2)}}\left(t ;-s_{\text {tot }}^{-}\right)$(red open circles) to first reach $-s_{\text {tot }}^{-}$given that it has not reached $s_{\text {tot }}^{+}$before. The data are from $10^{4}$ simulations of the Smoluchowski-Feynman ratchet using the same simulation parameters as in Fig. 7. (b) Passage probabilities for entropy production $\mathrm{P}_{+}^{(2)}$ (blue filled squares) to first reach the positive threshold $s_{\text {tot }}^{+}$given that it has not reached $-s_{\text {tot }}^{-}$before, and the passage probability for entropy production $\mathrm{P}_{-}^{(2)}$ (red filled circles) to first reach the positive threshold $-s_{\text {tot }}^{-}$ given that it has not reached $s_{\text {tot }}^{+}$before. We show the passage probabilities as a function of the negative threshold value $s_{\text {tot }}^{-}$; the positive threshold is $s_{\text {tot }}^{+}=2 s_{\text {tot }}^{-}$. The curves represent the analytical expressions for the passage probabilities given by Eqs. (5) and (6) $\left(P_{+}^{(2)}\right.$, blue solid curve; $P_{-}^{(2)}$, red dashed curve).

entropy-production passage probabilities $\mathrm{P}_{+}^{(2)}$ and $\mathrm{P}_{-}^{(2)}$ as a function of the value of the negative threshold $s_{\text {tot }}^{-}$. Simulation results are in accordance with the universal expressions given by Eqs. (5) and (6).

\section{Fluctuation theorem in waiting times}

We illustrate the waiting-time fluctuation theorem, given by Eq. (9) [or Eq. (73)], for the Smoluchowski-Feynman ratchet. We compute, using numerical simulations, the waiting times along forward trajectories I $\rightarrow$ II and backward trajectories II $\rightarrow$ I between two states characterized, respectively, by the coordinates $X=X_{\mathrm{I}}$ and $X=X_{\mathrm{II}}$, as illustrated in Fig. 10(a). In agreement with the fluctuation theorem for waiting times, we find that the normalized distribution $p_{T^{\mathrm{I} \rightarrow \mathrm{II}}}\left(t \mid s_{\text {env }}\right)$ is equal to the normalized distribution $p_{T_{-}^{\mathrm{II} \rightarrow 1}}\left(t \mid-s_{\text {env }}\right)$ [see Fig. 10(b)]. Here, the environment-entropy change is determined by the heat exchange between the system and the environment, i.e., $s_{\text {env }}=-Q / \mathrm{T}_{\text {env }}=F\left(X_{\text {II }}-X_{\mathrm{I}}\right) / \mathrm{T}_{\text {env }}+\left[V\left(X_{\mathrm{I}}\right)-V\left(X_{\mathrm{II}}\right)\right] /$ $\mathrm{T}_{\text {env }}$. In the inset of Fig. 10(b), we show simulation results for the ratio of passage probabilities, which is in agreement
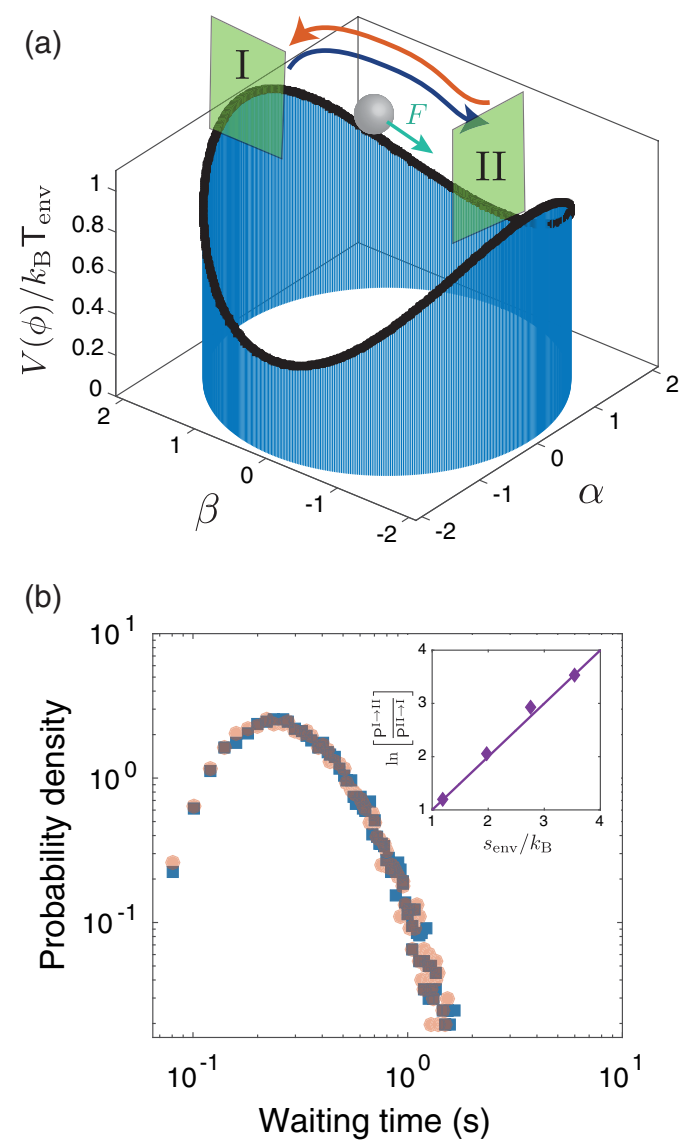

FIG. 10. Waiting-time statistics between the states $X_{\mathrm{I}}$ and $X_{\mathrm{II}}$ as illustrated in panel (a) for the Smoluchowski-Feynman ratchet with the potential (79). In panel (b), simulation results are shown for the normalized waiting-time distributions of transitions I $\rightarrow$ II (blue squares) and of reversed transitions II $\rightarrow$ I (red circles). The simulations are done with the same parameters as in Fig. 7, and the distributions are obtained from $10^{4}$ trajectories. Inset: Logarithm of the ratio between $\mathrm{P}_{+}^{\mathrm{I} \rightarrow \mathrm{II}}$ and $\mathrm{P}_{-}^{\mathrm{II} \rightarrow \mathrm{I}}$ as a function of the environment-entropy change in the transition I $\rightarrow$ II (magenta diamonds). The transition probability $\mathrm{P}_{+}^{\mathrm{I} \rightarrow \mathrm{II}}\left(\mathrm{P}_{-}^{\mathrm{II} \rightarrow \mathrm{I}}\right)$ is the fraction of the trajectories starting from $X_{\mathrm{I}}\left(X_{\mathrm{II}}\right)$ at $t=0$ that reach $X_{\mathrm{II}}\left(X_{\mathrm{I}}\right)$ at a later time $t>0$ without returning to $X_{\mathrm{I}}\left(X_{\mathrm{II}}\right)$ before. The solid line is a straight line of slope 1 . The data in the inset are obtained from $10^{5}$ simulations starting from state I and $10^{5}$ simulations starting from state II. 
with our theoretical result Eq. (75), i.e., $\mathrm{P}_{+}^{\mathrm{I} \rightarrow \mathrm{II}} / \mathrm{P}_{-}^{\mathrm{II} \rightarrow \mathrm{I}}=$ $e^{-Q / k_{\mathrm{B}} T_{\mathrm{env}}}$.

\section{APPLICATIONS TO ACTIVE MOLECULAR PROCESSES}

In contrast to the driven colloidal particles discussed in the last section, which are best viewed as continuous stochastic processes, many biochemical processes are often described in terms of transitions between discrete states. Examples are the motion of a molecular motor on a substrate with discrete binding sites or a chemical reaction that turns reactants into products. The statistics of waiting times of discrete processes can be obtained by a coarse-graining procedure of continuous processes, which we describe below. We apply our theory to a chemically driven hopping process with 1 degree of freedom and to the dynamics of RNA polymerases described by 2 degrees of freedom.

\section{A. From continuous to discrete processes}

We can apply the theory developed above to systems that progress in discrete steps using a coarse-graining procedure $[82,83]$. We consider a single continuous mesoscopic degree of freedom $X$, which can be used to describe a stochastic cyclic process. The variable $X$ can be interpreted as a generalized reaction coordinate or the position coordinate of a molecular motor, and it obeys the Langevin equation (76) with the same noise correlations. The effective potential $V(X)$ now describes the free energy profile along a chemical reaction, or a position coordinate, of a molecular motor.

We coarse grain the continuous variable $X$ by considering events when the process $X$ passes discrete points at positions $X_{M}$. The transition times between these points are examples of waiting times, similar to those in Kramers' theory [72]. An example is shown in Fig. 11, for which the points $X_{M}$ are located at the minima of the potential $V$. We introduce the transition times $T_{M \rightarrow M+1}$ when the process $X$ reaches the final state $X_{M+1}$ for the first time, starting from the initial state $X_{M}$, and given that the process $X$ has not reached $X_{M-1}$ before; we allow for several passages of the process $X$ through $X_{M}$. Similarly, we define $T_{M+1 \rightarrow M}$ for the reverse process.

The entropy change associated with a transition is $s_{\text {tot }}=F \ell / \mathrm{T}_{\text {env }}$, where we have used the fact that the process is cyclic. Entropy production is therefore related to position $X_{M}$ by

$$
S_{\mathrm{tot}}(t)=-N(t) \frac{F \ell}{\mathrm{T}_{\mathrm{env}}},
$$

where $N(t)=\left[X_{M}(0)-X_{M}(t)\right] / \ell$ is the number of steps in the negative direction minus the number of steps in the positive direction up to time $t$. The transition times are thus first-passage times of entropy production: $T_{M \rightarrow M+1}=T_{+}^{(2)}$

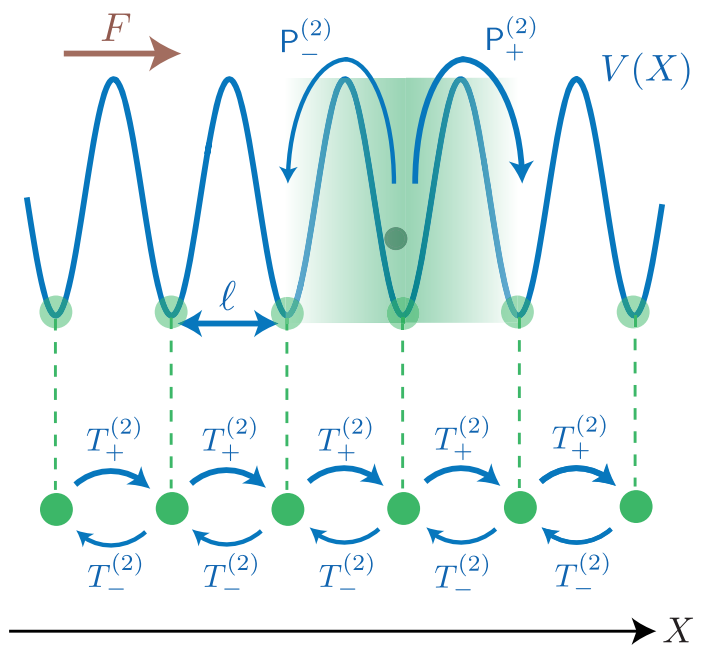

FIG. 11. Coarse-graining procedure from a continuous Langevin process (top) to a discrete Markov process (bottom). The horizonal axis denotes either a chemical coordinate, which quantifies the progress of a chemical reaction, or a position coordinate, which quantifies the position of a molecular motor. In the Langevin description, this coordinate is described by the position of a Brownian particle (gray circle) moving in a periodic potential $V(X)$ of period $\ell$ (blue curve) and driven by an external bias $F=\Delta G / \ell-F_{\text {mech }}$. In the discrete process, this coordinate is described by the state of a Markov jump process with stepping rates $k_{+}$and $k_{-}$, which are related to stopping times and passage probabilities of entropy production through $k_{+}=P_{+}^{(2)}\left\langle 1 / T_{+}^{(2)}\right\rangle$ and $k_{-}=P_{-}^{(2)}\left\langle 1 / T_{-}^{(2)}\right\rangle$. Our coarse-graining procedure corresponds to a partitioning of the coordinate axis into intervals (green shaded areas) centered on the minima of the potential (dashed lines).

and $T_{M+1 \rightarrow M}=T_{-}^{(2)}$, with thresholds $s_{\text {tot }}^{ \pm}=F \ell / T_{\text {env }}$. The probabilities for forward and backward hopping are the passage probabilities for entropy production $\mathrm{P}_{+}^{(2)}$ and $\mathrm{P}_{-}^{(2)}$. The hopping probabilities and the statistics of transition times therefore obey the universal equalities that we have derived in Secs. IV-VI. They can also be related to the usually discussed transition rates $k_{ \pm} \equiv \mathrm{P}_{ \pm}^{(2)}\left\langle 1 / T_{ \pm}^{(2)}\right\rangle$, which satisfy the condition of local detailed balance $k_{+} / k_{-}=$ $e^{F \ell / k_{\mathrm{B}} \mathrm{T}_{\mathrm{env}}}[40-42]$, as follows from Eqs. (65) and (69).

\section{B. Chemically driven hopping processes described by 1 degree of freedom}

Enzymes or molecular motors that are driven out of equilibrium by a chemical bias or an external force are examples of stepping processes. The thermodynamic bias of a chemical transition is often of the form $F \ell=\Delta G-F_{\text {mech }} \ell$, where $\Delta G$ denotes the chemical free energy change associated with the chemical transition and $F_{\text {mech }}$ is an externally applied force opposing motion driven by positive $\Delta G$.

We are interested in the statistics of chemical transition times $T_{M \rightarrow M^{\prime}}$, which are the first-passage times of entropy 
production $T_{+}^{(2)}$ and $T_{-}^{(2)}$. Equation (65) implies that the normalized distributions of $T_{+}^{(2)}$ and $T_{-}^{(2)}$ are identical. This symmetry condition of the distributions of forward and backward transition times could be tested experimentally. For instance, the relation $\left\langle T_{+}^{(2)}\right\rangle=\left\langle T_{-}^{(2)}\right\rangle$ on the mean dwell times could be tested experimentally for molecular motors that make reversible steps, for example, $\mathrm{F}_{0} \mathrm{~F}_{1}$-ATP synthases [84] and RNA polymerases in the backtracking state $[85,86]$.

We can also discuss the extreme-value statistics of the number of steps $N$ against the direction of the bias. We denote by $N_{\max }(t)>0$ the maximum number of steps against the direction of the bias, which is the most extreme fluctuation opposing the direction of the average current. The infimum law Eq. (1) implies an upper bound for the average of $N_{\max }(t)$ given by

$$
\left\langle N_{\max }(t)\right\rangle \leq \frac{k_{\mathrm{B}} \mathrm{T}_{\mathrm{env}}}{\Delta G-F_{\mathrm{mech}} \ell},
$$

for $\Delta G>F_{\text {mech }} \ell$. The right-hand side of Eq. (84) is the inverse of the Péclet number $\mathrm{Pe}=v \ell / D$. Moreover, Eq. (2) implies that the cumulative distribution of $N_{\max }(t)$ is bounded from above by an exponential

$$
\operatorname{Pr}\left(N_{\max }(t) \geq n\right) \leq e^{-n\left(\Delta G-F_{\text {mech }} \ell\right) / k_{\mathrm{B}} \mathrm{T}_{\mathrm{env}}}
$$

with $n \geq 0$. Equation (85) states that the probability that a molecular motor makes more than $n$ backsteps is smaller than or equal to $e^{-n\left(\Delta G-F_{\text {mech }} \ell\right) / k_{\mathrm{B}} \mathrm{T}_{\text {env }}}$. Therefore, our results on infima of entropy production constrain the maximum excursion of a molecular motor against the direction of an external force or a chemical bias.

\section{Dynamics of RNA polymerases: An example with 2 degrees of freedom}

We now apply the general results of our theory to a more complex example of a biomolecular process, which cannot be described in terms of a single degree of freedom, namely, the dynamics of RNA polymerases on a DNA template. RNA polymerases transcribe genetic information from DNA into RNA. During transcription, RNA polymerases adopt two different states: the elongating state and the backtracking state [85]. Elongation is an active process where RNA polymerases move stepwise and unidirectionally along the DNA while polymerizing a complementary RNA driven by the chemical free energy of the incorporated nucleotides, as illustrated in Fig. 12(a). In the elongating state, the motion of RNA polymerase and the polymerization reaction are fueled by the hydrolysis of ribonucleotide triphosphate (NTP), which provides the free energy $\Delta G_{\mathrm{NTP}}$ per nucleotide [87]. Backtracking is a passive motion of the RNA polymerase on the DNA template that displaces the RNA $3^{\prime}$ end from the active site (a) Elongation

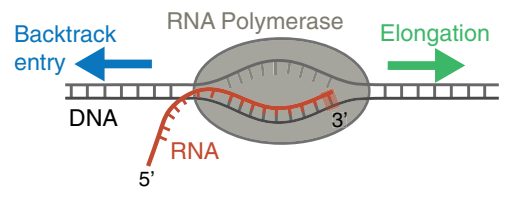

(b) Backtracking

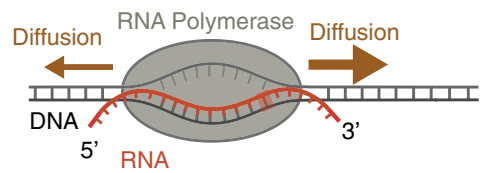

(c)

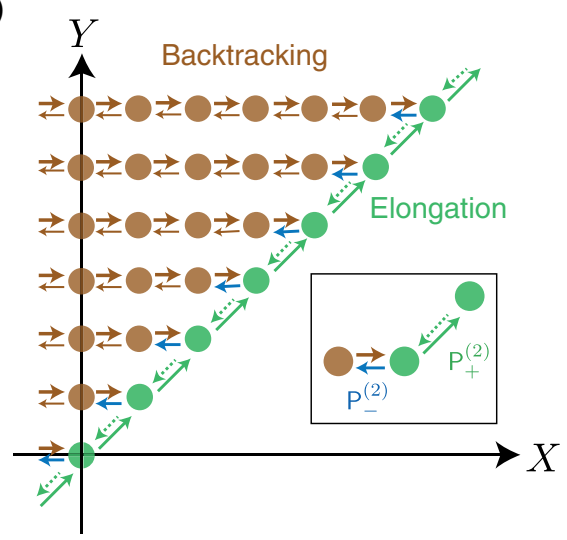

FIG. 12. Stochastic model for transcription. (a) Sketch of an elongating RNA polymerase. The polymerase can either incorporate a new nucleotide in its active site (red shaded square) or enter a backtracked state. (b) Sketch of a backtracking RNA polymerase. The polymerase diffuses along the DNA template until its active site (red shaded area) aligns with the 3' end of the transcribed RNA. (c) Network representation of a Markov jump process describing the dynamics of RNA polymerases. The $X$ coordinate gives the position of the polymerase along the DNA template, and the $Y$ coordinate gives the number of nucleotides transcribed. Green nodes represent elongating states, and brown nodes represent backtracked states. The inset shows all possible transitions from or to an elongating state. The green solid arrow denotes active translocation of the RNA polymerase, and the dashed green arrow denotes its time-reversed transition. Because the heat dissipated during the forward translocation is much larger than $k_{\mathrm{B}} \mathrm{T}_{\text {env }}$, the rate of the backward transition is very small. The blue arrow corresponds to the entry in a backtracked state, and the brown arrow is the exit from a backtracked state into the elongating state. In the backtracked state, motion is biased towards the elongating state.

of the polymerase and leaves the enzyme transcriptionally inactive [88], as illustrated in Fig. 12(b). Transcription is thus an active polymerization process that is interspersed by pauses of passive stepping motion.

The main properties of the dynamics of RNA polymerases are the following: In the elongating state, RNA polymerases can either continue elongation [green arrows in Figs. 12(a) and 12(c)] or enter a backtracking state [blue 
arrows in Figs. 12(a) and 12(c)]. A RNA polymerase in the backtracking state diffuses on the DNA template until its active site is realigned with the $3^{\prime}$ end of the RNA $[85,89]$. This diffusive motion is often biased by either the presence of external opposing forces $F_{\text {mech }}$ or by an energy barrier $\Delta V_{\mathrm{RNA}}$ related to the secondary structure of the nascent RNA [91,92]; see Fig. 13.

The dynamics of RNA polymerase can thus be described as a continuous-time Markov jump process on a twodimensional network [92-94]; see Fig. 12(c). The state of a polymerase is determined by two variables: $X$ denotes the position of the polymerase (in nucleotides) along the DNA template, and $Y$ denotes the number of NTP molecules hydrolyzed during elongation. When $X=Y$, the polymerase is in the elongating state, and when $X<Y$, the polymerase is in the backtracked state. The variable $N=Y-X$ denotes the depth of the backtrack. We consider, for simplicity, a stationary process with steady-state distribution $p_{\mathrm{ss}}(X, Y)$ on a homogeneous DNA template. Therefore, the steady-state probability to find the polymerase in an elongating state is $p_{\mathrm{ss}}(0)=p_{\mathrm{ss}}(X, X)$, and the steady-state probability to find the polymerase backtracked by $N$ steps is $p_{\text {ss }}(N)=p_{\text {ss }}(X, X+N)$.

Two relevant quantities in transcription are the pause density $[85,90,93,95]$, i.e., the probability per nucleotide that an elongating polymerase enters a backtracked state, and the maximum depth of a backtrack $[85,86]$, i.e., the maximum number of backtracked nucleotides. Our theory provides expressions for the statistics of these quantities in terms of infima and stopping-time statistics of entropy production.

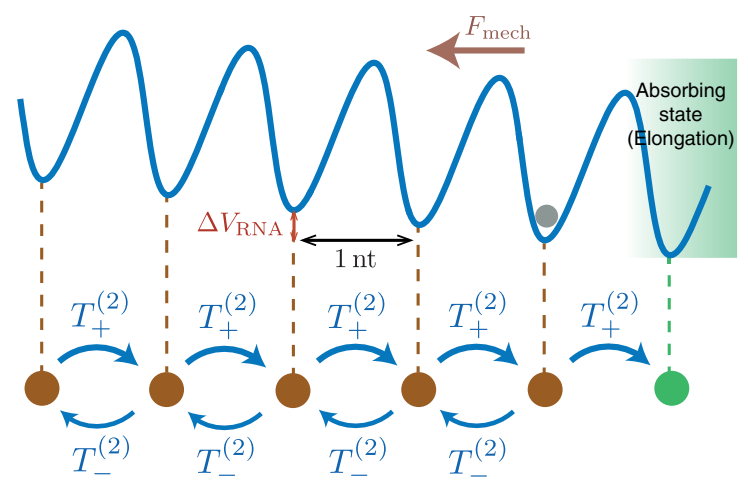

FIG. 13. The dynamics of RNA polymerase during backtracking. The motion of the polymerase is represented as a diffusion process in a tilted periodic potential with an absorbing boundary on the right (which corresponds to the transition from the backtracked state into the elongating state). The period of the potential is equal to the length of one nucleotide (nt). The potential is tilted towards the absorbing boundary due to an energetic barrier $\Delta V_{\mathrm{RNA}}$, and an external opposing mechanical force $F_{\text {mech }}$ pushes the polymerase away from the absorbing boundary. This process can be coarse grained into a discrete onedimensional hopping process with waiting times $T_{+}^{(2)}$ and $T_{-}^{(2)}$.
If the probability flux from the elongating state into the backtracked state is smaller than the flux of the reverse process, which implies that entropy $S_{\text {tot }}$ reduces when the polymerase enters a backtrack, the pause density is equal to a passage probability $\mathrm{P}_{-}^{(2)}$ of entropy production. In addition, we consider that an elongating polymerase only moves forward. These conditions are necessary for backtrack entry to correspond to a first-passage process of entropy production with two absorbing boundaries of different sign. The probability $\mathrm{P}_{+}^{(2)}$ then corresponds to the probability for the polymerase to attach a new nucleotide, as illustrated in the inset in Fig. 12(c). These probabilities obey Eqs. (5) and (6) [96], with $-s_{\text {tot }}^{-}=$ $\Delta S_{\text {be }}+F_{\text {mech }} \ell / \mathrm{T}_{\text {env }}$ and $s_{\text {tot }}^{+}=\left(\Delta G_{\mathrm{NTP}}-F_{\text {mech }} \ell\right) / \mathrm{T}_{\text {env }}$. Here, $\Delta S_{\mathrm{be}}=-k_{\mathrm{B}} \ln \left[p_{\mathrm{ss}}(1) / p_{\mathrm{ss}}(0)\right]$ is the system entropy change when entering the backtrack. If $\Delta G_{\mathrm{NTP}} /$ $k_{\mathrm{B}} \mathrm{T}_{\text {env }} \gg 1$, which holds in typical experiments [93], we have $e^{-s_{\mathrm{tot}}^{+} / k_{\mathrm{B}}} \ll 1$, and thus we find, using Eqs. (51)-(52), simple expressions for the pause densities and the probability to continue elongation,

$$
\begin{gathered}
\mathrm{P}_{+}^{(2)} \simeq 1-e^{\left(\mathrm{T}_{\text {env }} \Delta S_{\text {be }}+F_{\text {mech }} \ell\right) / k_{\mathrm{B}} \mathrm{T}_{\text {env }}}, \\
\mathrm{P}_{-}^{(2)} \simeq e^{\left(\mathrm{T}_{\text {env }} \Delta S_{\text {be }}+F_{\text {mech }} \ell\right) / k_{\mathrm{B}} \mathrm{T}_{\text {env }}} .
\end{gathered}
$$

The backtrack entry of a polymerase, and therefore the pause density, is thus determined by a kinetic competition between an entropic term $\mathrm{T}_{\text {env }} \Delta S_{\text {be }}<0$ and the work done by the force acting on the polymerase, $F_{\text {mech }} \ell>0$.

We can also discuss the dynamics of backtracked RNA polymerases. During a backtrack, the dynamics of RNA polymerase is captured by a one-dimensional biased diffusion process with an absorbing boundary corresponding to the transition to elongation [86]; see Fig. 13. During backtracking, the bias $F=\Delta V_{\mathrm{RNA}} / \ell-F_{\text {mech }}$, where $\Delta V_{\mathrm{RNA}}$ is an energy barrier associated with the secondary structure of the nascent RNA [90-92]. The waiting times of the corresponding Markov jump process are equal to firstpassage times of entropy production. For a polymerase with backtrack depth $N$, the waiting time to decrease $N$ by one $T_{+}^{(2)}(N)$ and the waiting time to increase $N$ by one $T_{-}^{(2)}(N)$ are first-passage times of entropy production with two absorbing boundaries $s_{\text {tot }}^{+}=-k_{\mathrm{B}} \ln \left[p_{\mathrm{ss}}(N-1) / p_{\mathrm{ss}}(N)\right]+$ $F \ell / \mathrm{T}_{\text {env }}$ and $-s_{\text {tot }}^{-}=-k_{\mathrm{B}} \ln \left[p_{\mathrm{ss}}(N+1) / p_{\mathrm{ss}}(N)\right]-F \ell / \mathrm{T}_{\text {env }}$. If the bias dominates, the two boundaries have opposite sign, and we can use the theory of stopping times developed here. The hopping probabilities for forward and backward steps in the backtrack then follow from our general expressions for passage probabilities of entropy production, Eqs. (5) and (6).

The maximum backtrack depth $N_{\max }(t)$, at a time $t$ after entering the backtrack state, is related to the infimum of entropy production, $S_{\text {inf }}(t)$. The infimum law (1) therefore provides a thermodynamic bound on the average of the 
maximum extent of a backtrack. Using Eq. (84) with $\Delta V_{\mathrm{RNA}} \simeq 0.5 k_{\mathrm{B}} \mathrm{T}_{\text {env }}$ and $F_{\text {mech }} \ell \simeq 0.4 k_{\mathrm{B}} \mathrm{T}_{\text {env }}$ [90-92], we estimate the upper bound for the maximum backtrack depth $\left\langle N_{\max }(t)\right\rangle \lesssim 10$ nucleotides; we have used the parameter values $F_{\text {mech }}=5 \mathrm{pN}, k_{\mathrm{B}} \mathrm{T}_{\text {env }}=4 \mathrm{pN} \mathrm{nm}$, and $\ell=0.34 \mathrm{~nm}$ (the distance between two nucleotides). Similarly, from Eq. (85), we find that the cumulative distribution of the maximum backtrack depth is upper bounded by an exponential [97]. This is consistent with single molecule experiments on RNA polymerase backtracking, which have reported that the distribution of the maximum backtrack depth is exponential (see Fig. S3 in Ref. [85]).

\section{DISCUSSION}

The second law of thermodynamics is a statement about the average entropy production when many realizations of a mesoscopic system are considered. This fundamental law leaves open the question of whether fluctuations of entropy production also obey general laws [37-39,47,78,98-101]. In the present paper, we have demonstrated that the infimum of entropy production and stopping times of entropy production exhibit statistical features that are universal and do not depend on the physical nature of a given system. The simplest example that illustrates these universal features is the case where entropy production follows a stochastic drift-diffusion process described by the Langevin equation

$$
\frac{\mathrm{d} S_{\mathrm{tot}}(t)}{\mathrm{d} t}=v_{\mathrm{S}}+\eta_{\mathrm{S}}(t)
$$

where the constant drift velocity $v_{\mathrm{S}}>0$ corresponds to the average entropy production rate. The Gaussian white noise $\eta_{\mathrm{S}}(t)$ has zero mean, and the autocorrelation $\left\langle\eta_{\mathrm{S}}(t) \eta_{\mathrm{S}}(0)\right\rangle=2 D_{\mathrm{S}} \delta(t)$. The diffusion constant $D_{\mathrm{S}}$ characterizes entropy-production fluctuations and obeys

$$
D_{\mathrm{S}}=k_{\mathrm{B}} v_{\mathrm{S}}
$$

Equations (88) and (89) can be derived from Eq. (78). These equations exhibit all the universal properties of entropy production discussed in this paper; see Sec. VII.

The infimum of entropy production up to a given time must be either zero or negative, even though entropy production is always positive on average. Here, we have shown that when averaging over all realizations in a nonequilibrium steady state, the average infimum of entropy production is always greater than or equal to $-k_{\mathrm{B}}$. Furthermore, the global infimum of entropy production is exponentially distributed with mean $-k_{\mathrm{B}}$. This is an exact result for the distribution of global infima of a correlated stochastic process, which is interesting because only a few similar results are known [102-104].
Our results are of special interest for experiments because entropy production reaches its global infimum in a finite time. The exact results on entropy infima could be verified experimentally, for example, using colloidal particles trapped with optical tweezers $[75,76,105]$ and feedback traps [106]. Other experimental systems that could be used to test our results are single-molecule experiments $[84,107,108]$ or mesoscopic electronic systems such as single-electron boxes [36].

We have furthermore shown that the stopping-time statistics and the passage statistics of entropy production exhibit universality. We have found a remarkable symmetry for the stopping times of entropy production. In a network of states, this symmetry relation implies that, for each link between two states, the statistics of waiting times is the same for forward and backward jumps along this link. Measuring statistics of waiting times thus reveals whether two forward and backward transitions along a link in a network of states are each other's time reverse. If the corresponding waiting-time distributions are not equal, then forward and backward transitions are not related by time reversal. It would be interesting to relate this symmetry for the waiting-time distributions to other symmetry properties of stochastic processes, such as the conditional reversibility relations [109].

Our work is based on the finding that in a steady state, $e^{-S_{\text {tot }}(t) / k_{\mathrm{B}}}$ is a martingale process. A martingale is a process for which the mean is unpredictable, or equivalently, it represents a fair game with no net gain or loss. The theory of martingales is well developed because of its importance to fields such as quantitative finance $[59,60]$ and decision theory [110]. In stochastic thermodynamics, martingales have not yet attracted much attention. However, the martingale property and equations of the form (21) and (23) have been discussed in the context of action functionals in Ref. [26]. Our present work explores the martingale property of $e^{-S_{\text {tot }}(t) / k_{\mathrm{B}}}$, revealing several previously unknown properties of stochastic entropy production of stationary processes. We show that entropy production itself is a submartingale, and we relate this fact to the second law of thermodynamics. Remarkably, the universal statistics of infima and stopping times all follow from the martingale property of $e^{-S_{\text {tot }}(t) / k_{\mathrm{B}}}$. Our work thus highlights the relevance of martingales to nonequilibrium thermodynamics.

Our results on entropy production fluctuations provide expressions for the hopping probabilities, the statistics of waiting times, and the extreme-value statistics of active molecular processes. We have illustrated our results on active molecular processes described by 1 degree of freedom and on the stochastic dynamics of DNA transcription by RNA polymerases. Our theory provides expressions for the probability of molecular motors to step forward or backward in terms of the entropy produced during the forward and backward steps, and it relates waiting-time 
statistics of forward and backward transitions. Moreover, the infimum law provides a thermodynamic bound on the maximal excursion of a motor against the effective force that drives its motion. For the dynamics of RNA polymerases, this implies that the statistics of the maximum backtrack depth is bounded by a limiting exponential distribution. This provides predictions about the mean and the second moment of backtrack depths that could be tested in future single-molecule experiments.

Cells and proteins often execute complex functions at random times. Stopping times provide a powerful tool to characterize the timing of stochastic biological processes. It will be interesting to explore whether our approach to the statistics of stopping times of nonequilibrium processes is also relevant to more complex systems, such as flagellar motor switching [111], sensory systems [112-115], selfreplicating nucleic acids [116,117], or cell-fate decisions [118-120].

\section{ACKNOWLEDGMENTS}

We thank Andre Cardoso Barato, Meik Dörpinghaus, Shamik Gupta, Stephan W. Grill, Mostafa Khalili-Marandi, Ana Lisica, Heinrich Meyr, Juan M. R. Parrondo, Simone Pigolotti, Matteo Polettini, Madan Rao, Lawrence S. Schulman, and Udo Seifert for stimulating discussions. We thank Raphael Chetrite for carefully reading our manuscript. E. R. acknowledges funding from the Spanish Government, Grant TerMic (No. FIS2014-52486-R).

\section{APPENDIX A: MARTINGALE PROCESSES}

We define martingale processes and discuss their properties using concepts and notations from probability and martingale theory [53].

\section{Definition of a martingale process}

A sequence of random variables $X_{1}, X_{2}, X_{3}, \ldots$ is a martingale when $\mathbb{E}\left(\left|X_{n}\right|\right)<\infty$, for all $n \in \mathbb{N}$, and when the conditional expectation $\mathbb{E}\left(X_{n} \mid X_{1}, X_{2}, \ldots, X_{m}\right)=X_{m}$, for all $m \leq n$. A martingale is thus a process for which the mean is unpredictable, or equivalently, it represents a fair game with no net gain or loss. Notice that here we use the standard notation $\mathbb{E}_{\mathbb{P}}\left(X_{n}\right)=\left\langle X_{n}\right\rangle_{\mathbb{P}}$ for the expectation of a random variable $X_{n}$ with respect to the measure $\mathbb{P}$.

For our purposes, we also need a general definition of a martingale process with respect to a filtered probability space. We consider a stochastic process $X(\omega ; t)$, with $t \in[0, \infty)$, and a filtered probability space $\left(\Omega, \mathcal{F},\{\mathcal{F}(t)\}_{t \geq 0}, \mathbb{P}\right)$. For processes in continuous time, we consider that the filtration $\{\mathcal{F}(t)\}_{t \geq 0}$ and the process $X$ are right continuous. A process $X(\omega ; t)$ is a martingale with respect to the filtered probability space $\left(\Omega, \mathcal{F},\{\mathcal{F}(t)\}_{t \geq 0}, \mathbb{P}\right)$ when the following occur: $X$ is adapted to the filtration $\{\mathcal{F}(t)\}_{t \geq 0} ; X$ is integrable, i.e., $\mathbb{E}(|X(\omega, t)|)<\infty$; and the conditional expectation
$\mathbb{E}(X(\omega ; t) \mid \mathcal{F}(s))=X(\omega ; s)$ for all $s<t$. The latter condition is equivalent to

$$
\int_{\omega \in \Phi} X(\omega ; s) \mathrm{d} \mathbb{P}=\int_{\omega \in \Phi} X(\omega ; t) \mathrm{d} \mathbb{P},
$$

for any $s<t$ and for any $\Phi \in \mathcal{F}(s)$. A submartingale satisfies the inequality

$$
\int_{\omega \in \Phi} X(\omega ; s) \mathrm{d} \mathbb{P} \leq \int_{\omega \in \Phi} X(\omega ; t) \mathrm{d} \mathbb{P}
$$

for any $s<t$ and for any $\Phi \in \mathcal{F}(s)$. The Wiener process, also called a Brownian motion, is an example of a martingale process.

\section{Uniform integrability}

Uniformly integrable processes play an important role in martingale theory. We call a stochastic process $X$ uniform integrable on the probability space $\left[\Omega,(\mathcal{F}(t))_{t \geq 0}\right.$, $\mathcal{F}, \mathbb{P}]$ if given $\epsilon>0$, there exists a $K \in[0, \infty)$ such that

$$
\mathbb{E}_{\mathbb{P}}\left(|X(t)| I_{|X(t)|>K}\right)<\epsilon,
$$

for all $t \geq 0$. The indicator function $I_{|X(t)|>K}$ is defined as

$$
I_{|X(t)|>K}= \begin{cases}1 & \text { if }|X(t)|>K, \\ 0 & \text { if }|X(t)| \leq K .\end{cases}
$$

A bounded random process is uniformly integrable, and a uniformly integrable process is integrable, i.e., $\mathbb{E}(|X(t)|)<\infty$ for all values of $t$. Uniform integrability can thus be seen as a condition that is less stringent than boundedness but more stringent than integrability.

For a uniformly integrable martingale process, the random variable $X(\omega ; \infty)=\lim _{t \rightarrow \infty} X(\omega ; t)$ exists [53], and it is finite for $\mathbb{P}$-almost all $\omega \in \Omega$. A process is uniform integrable if and only if [53]

$$
X(\omega ; t)=\mathbb{E}(X(\omega ;+\infty) \mid \mathcal{F}(t)),
$$

for $t \geq 0$, and with $X(\omega ;+\infty)$ integrable, i.e., $\mathbb{E}(|X(\omega ;+\infty)|)<\infty$.

\section{Doob's maximal inequality}

Doob's maximal inequality provides an upper bound on the cumulative distribution of the supremum of a nonnegative submartingale process $X(\omega ; \tau)$ in a time interval $[0, t][43]$, viz.,

$$
\operatorname{Pr}\left(\sup _{\tau \in[0, t]} X(\omega ; \tau) \geq \lambda\right) \leq \frac{1}{\lambda} \mathbb{E}_{\mathbb{P}}(X(\omega ; t)),
$$


for any constant $\lambda>0$. Doob's maximal inequality, given by Eq. (A6), holds for non-negative submartingales in discrete time and for right-continuous non-negative submartingales in continuous time [53].

Note that Doob's maximal inequality is a unique property of martingales and a stronger result than Markov's inequality. Markov's inequality provides an upper bound on the cumulative distribution of a nonnegative random variable $X$ :

$$
\operatorname{Pr}(X(\omega ; t) \geq \lambda) \leq \frac{1}{\lambda} \mathbb{E}_{\mathbb{P}}(X(\omega ; t)),
$$

with $\lambda>0$. Since $\operatorname{Pr}(X(\omega ; t) \geq \lambda) \geq \operatorname{Pr}\left(\sup _{\tau \in[0, t]} X(\omega ; \tau) \geq \lambda\right)$, Markov's inequality does not imply Doob's maximal inequality, but Doob's maximal inequality does imply Markov's inequality for non-negative martingales.

\section{Doob's optional sampling theorem}

We consider a uniformly integrable martingale process $X(\omega ; t)$ adapted to the filtration $\{\mathcal{F}(t)\}_{t \geq 0}$, and two stopping times $T_{1}(\omega)$ and $T_{2}(\omega)$, with the property that for each $\omega \in \Omega, T_{1}(\omega) \leq T_{2}(\omega)$. Additionally, in continuous time, $X$ is right continuous. Doob's optional sampling theorem then states that $[53,55]$

$$
\int_{\omega \in \Phi} X\left(\omega ; T_{2}(\omega)\right) \mathrm{d} \mathbb{P}=\int_{\omega \in \Phi} X\left(\omega ; T_{1}(\omega)\right) \mathrm{d} \mathbb{P},
$$

for each set $\Phi \in \mathcal{F}\left(T_{1}\right)$. The sub- $\sigma$-algebra $\mathcal{F}\left(T_{1}\right)$, corresponding to the stopping time $T_{1}$, is defined as

$$
\mathcal{F}\left(T_{1}\right)=\left\{\Phi \subset \Omega: \forall t \geq 0, \Phi \cap\left\{\omega: T_{1}(\omega) \leq t\right\} \in \mathcal{F}(t)\right\} .
$$

\section{APPENDIX B: PROPERTIES OF STOCHASTIC ENTROPY PRODUCTION}

We discuss the mathematical properties of the stochastic entropy production defined in Eq. (11). We first show that entropy production is a process of odd parity with respect to the time-inversion operator $\Theta_{t}$. Second, we show that $e^{-S_{\text {tot }}(\omega, t) / k_{\mathrm{B}}}$ is a uniformly integrable martingale with respect to the filtered probability space $\left(\Omega, \mathcal{F},\{\mathcal{F}(t)\}_{t \geq 0}, \mathbb{P}\right)$ generated by the dynamics of the mesoscopic degrees of freedom. This is a key property of entropy production, and it allows us to apply the techniques of martingale processes to entropy production.

\section{Entropy production is odd under time reversal}

An interesting property of entropy production is the change of its sign under the time-reversal operation $\Theta_{t}=\mathrm{T}_{t} \circ \Theta$, which time reverses trajectories with respect to the reference time $t / 2$, viz.,

$$
\begin{aligned}
S_{\text {tot }}\left(\Theta_{t}(\omega) ; t\right) & =k_{\mathrm{B}} \ln \frac{\left.\mathrm{d} \mathbb{P}\right|_{\mathcal{F}(t)}}{\left.\mathrm{d}(\mathbb{P} \circ \Theta)\right|_{\mathcal{F}(t)}}\left(\Theta_{t}(\omega)\right) \\
& =k_{\mathrm{B}} \ln \frac{\left.\mathrm{d}\left(\mathbb{P} \circ \Theta_{t}\right)\right|_{\mathcal{F}(t)}}{\left.\mathrm{d}\left(\mathbb{P} \circ \Theta \circ \Theta_{t}\right)\right|_{\mathcal{F}(t)}}(\omega) \\
& =k_{\mathrm{B}} \ln \frac{\left.\mathrm{d}\left(\mathbb{P} \circ \mathrm{T}_{t} \circ \Theta\right)\right|_{\mathcal{F}(t)}}{\left.\mathrm{d}\left(\mathbb{P} \circ \Theta \circ \mathrm{T}_{t} \circ \Theta\right)\right|_{\mathcal{F}(t)}}(\omega) \\
& =k_{\mathrm{B}} \ln \frac{\left.\mathrm{d}(\mathbb{P} \circ \Theta)\right|_{\mathcal{F}(t)}}{\left.\mathrm{d} \mathbb{P}\right|_{\mathcal{F}(t)}}(\omega) \\
& =-S_{\text {tot }}(\omega ; t),
\end{aligned}
$$

where we have used $\mathbb{P} \circ \mathrm{T}_{t}=\mathbb{P}, \mathbb{P} \circ \Theta \circ \mathrm{T}_{t}=\mathbb{P} \circ \Theta$, and $\Theta=\Theta^{-1}$.

\section{The exponentiated negative entropy production is a uniformly integrable martingale in a steady state}

Here, we first show that in a steady state, the exponential $e^{-S_{\text {tot }}(\omega ; t) / k_{\mathrm{B}}}$ is a martingale. We then show that it is also uniformly integrable. Both properties follow from the fact that $e^{-S_{\text {tot }}(\omega ; t) / k_{\mathrm{B}}}$ is a Radon-Nikodým density process. Indeed, the process $e^{-S_{\text {tot }}(\omega ; t) / k_{\mathrm{B}}}$ is the Radon-Nikodým density process of the filtered probability space $\left(\Omega, \mathcal{F},\{\mathcal{F}(t)\}_{t \geq 0}, \mathbb{Q}\right)$ with respect to the filtered probability space $\left(\Omega, \mathcal{F},\{\mathcal{F}(t)\}_{t \geq 0}, \mathbb{P}\right)$ :

$$
e^{-S_{\text {tot }}(\omega ; t) / k_{\mathrm{B}}}=\frac{\left.\mathrm{d} \mathbb{Q}\right|_{\mathcal{F}(t)}}{\left.\mathrm{d} \mathbb{P}\right|_{\mathcal{F}(t)}}(\omega),
$$

with $\mathbb{Q}=\mathbb{P} \circ \Theta$, the time-reversed measure, and $t \geq 0$. Consider two sub- $\sigma$-algebras $\mathcal{F}(\tau)$ and $\mathcal{F}(t)$ of $\mathcal{F}$, with $\tau<t$. We first write the measure $\mathbb{Q}(\Phi)$ of a set $\Phi \in \mathcal{F}(\tau)$ as an integral over the set $\Phi$ with respect to the probability space $\left(\Omega, \mathcal{F}(\tau),\left.\mathbb{Q}\right|_{\mathcal{F}(\tau)}\right)$,

$$
\begin{aligned}
\mathbb{Q}(\Phi) & =\left.\int_{\omega \in \Phi} \mathrm{d} \mathbb{Q}\right|_{\mathcal{F}(\tau)} \\
& =\left.\int_{\omega \in \Phi} e^{-S_{\text {tot }}(\omega ; \tau) / k_{\mathrm{B}}} \mathrm{d} \mathbb{P}\right|_{\mathcal{F}(\tau)} \\
& =\int_{\omega \in \Phi} e^{-S_{\text {tot }}(\omega ; \tau) / k_{\mathrm{B}}} \mathrm{d} \mathbb{P} .
\end{aligned}
$$

Alternatively, we write the measure $\mathbb{Q}(\Phi)$ as an integral over the set $\Phi$ with respect to the probability space $\left(\Omega, \mathcal{F}(t),\left.\mathbb{Q}\right|_{\mathcal{F}(t)}\right)$,

$$
\begin{aligned}
\mathbb{Q}(\Phi) & =\left.\int_{\omega \in \Phi} \mathrm{d} \mathbb{Q}\right|_{\mathcal{F}(t)} \\
& =\left.\int_{\omega \in \Phi} e^{-S_{\text {tot }}(\omega ; t) / k_{\mathrm{B}}} \mathrm{d} \mathbb{P}\right|_{\mathcal{F}(t)} \\
& =\int_{\omega \in \Phi} e^{-S_{\text {tot }}(\omega ; t) / k_{\mathrm{B}}} \mathrm{d} \mathbb{P} .
\end{aligned}
$$


In Eqs. (B3) and (B4), we use that, for $\tau<t$, by definition, $\left.\mathbb{P}\right|_{\mathcal{F}(\tau)}(\Phi)=\left.\mathbb{P}\right|_{\mathcal{F}(t)}(\Phi)=\mathbb{P}(\Phi)$ for all sets $\Phi \in \mathcal{F}(\tau)$. We thus have the equality

$$
\int_{\omega \in \Phi} e^{-S_{\text {tot }}(\omega ; \tau) / k_{\mathrm{B}}} \mathrm{d} \mathbb{P}=\int_{\omega \in \Phi} e^{-S_{\text {tot }}(\omega ; t) / k_{\mathrm{B}}} \mathrm{d} \mathbb{P},
$$

for all sets $\Phi \in \mathcal{F}(\tau)$, which is identical to Eq. (A1), which defines a martingale process. The process $e^{-S_{\text {tot }}(\omega ; t) / k_{\mathrm{B}}}$ is therefore a martingale process with respect to the measure $\mathbb{P}$. Equation (B5) is an example of the martingale property of a stochastic process. Other examples of martingales have previously been discussed in the context of action functionals [26].

We also show that $e^{-S_{\mathrm{tot}}(\omega ; t) / k_{\mathrm{B}}}$ is uniformly integrable. We use the necessary and sufficient condition (A5) for uniform integrability. The process $e^{-S_{\text {tot }}(\omega ; t) / k_{\mathrm{B}}}$ is uniformly integrable since, by definition, $e^{-S_{\text {tot }}(\omega ; t) / k_{\mathrm{B}}}$ is a conditional expectation:

$$
e^{-S_{\mathrm{tot}}(\omega ; t) / k_{\mathrm{B}}}=\mathbb{E}\left(\frac{\mathrm{d} \mathbb{P} \circ \Theta}{\mathrm{d} \mathbb{P}}(\omega) \mid \mathcal{F}(t)\right),
$$

with $\frac{\mathrm{d} \mathbb{P} \circ \Theta}{d \mathbb{P}}(\omega)=e^{-S_{\text {tot }}(\omega ;+\infty) / k_{\mathrm{B}}}$ a positive and integrable random variable.

\section{APPENDIX C: STOCHASTIC DOMINANCE}

Consider two positive-valued random variables $X \geq 0$ and $Y \geq 0$. We define their cumulative distributions as

$$
\begin{aligned}
& F_{X}(x)=\operatorname{Pr}(X \leq x), \\
& F_{Y}(x)=\operatorname{Pr}(Y \leq x) .
\end{aligned}
$$

We say that $X$ dominates $Y$ stochastically when the cumulative distribution functions of $X$ and $Y$ satisfy the relation $F_{X}(x) \geq F_{Y}(x)$. If $X$ dominates $Y$ stochastically, then the mean value of $X$ is smaller than the mean value of $Y:\langle X\rangle \leq\langle Y\rangle$. This follows directly from the relation $\langle X\rangle=$ $\int_{0}^{\infty} \mathrm{d} x\left[1-F_{X}(x)\right]$ between the mean and the cumulative distribution.

\section{APPENDIX D: GENERALIZED PASSAGE PROBABILITIES FOR ENTROPY PRODUCTION}

We generalize the relations Eqs. (45) and (46) for the two-boundary passage probabilities of entropy production in order to account for trajectories starting from a given macrostate I. We define a macrostate I as a subset of the phase space $\left\{\boldsymbol{q}, \boldsymbol{q}^{*}\right\}$. Here, we consider macrostates defined by a given set of constraints on the variables of even parity $\mathrm{I}_{\text {even }} \subseteq \mathbb{R}^{n}$. The initial state $\omega(0)$ belongs to the macrostate I if $\boldsymbol{q}(0) \in \mathrm{I}_{\text {even }}$.

We define the passage probability $\mathrm{P}_{+. \mathrm{I}}^{(2)}$ (and $\mathrm{P}_{-\mathrm{I}}^{(2)}$ ) as the joint probability of the process being initially in the macrostate I, i.e., $\omega(0) \in \mathrm{I}$, and of entropy production to pass the threshold $s_{\text {tot }}^{+}\left(s_{\text {tot }}^{-}\right)$before passing the threshold $s_{\text {tot }}^{-}\left(s_{\text {tot }}^{+}\right)$. Formally, these passage probabilities are defined by

$$
\begin{aligned}
& P_{+, \mathrm{I}}^{(2)} \equiv \mathbb{P}\left(\Phi_{+} \cap \Gamma_{\mathrm{I}}\right), \\
& \mathbf{P}_{-, \mathrm{I}}^{(2)} \equiv \mathbb{P}\left(\Phi_{-} \cap \Gamma_{\mathrm{I}}\right),
\end{aligned}
$$

with $\Phi_{+}$and $\Phi_{-}$the sets defined in Eqs. (31) and (32), respectively, and $\Gamma_{\mathrm{I}}$ the set of trajectories starting from the macrostate I:

$$
\Gamma_{\mathrm{I}}=\{\omega \in \Omega: \omega(0) \in \mathrm{I}\} .
$$

We also define the conjugate probabilities $\tilde{\mathrm{P}}_{+, \mathrm{I}}^{(2)}$ and $\tilde{\mathrm{P}}_{-, \mathrm{I}}^{(2)}$ :

$$
\begin{aligned}
& \tilde{\mathrm{P}}_{+, \mathrm{I}}^{(2)} \equiv(\mathbb{P} \circ \Theta)\left(\Phi_{+} \cap \Gamma_{\mathrm{I}}\right), \\
& \tilde{\mathrm{P}}_{-, \mathrm{I}}^{(2)} \equiv(\mathbb{P} \circ \Theta)\left(\Phi_{-} \cap \Gamma_{\mathrm{I}}\right) .
\end{aligned}
$$

Here, we have used $\mathrm{I}=\tilde{\mathrm{I}}$ since we have defined the macrostate using constraints on variables of even parity only.

For a nonequilibrium steady-state process, i.e., $\left\langle S_{\mathrm{tot}}(t)\right\rangle>0, S_{\mathrm{tot}}(t)$ passes one of the two thresholds $s_{\mathrm{tot}}^{ \pm}$ in a finite time, with probability 1 . We thus have

$$
\begin{aligned}
& \mathrm{P}_{+, \mathrm{I}}^{(2)}+\mathrm{P}_{-, \mathrm{I}}^{(2)}=\mathbb{P}\left(\Gamma_{\mathrm{I}}\right), \\
& \tilde{\mathrm{P}}_{+, \mathrm{I}}^{(2)}+\tilde{\mathrm{P}}_{-, \mathrm{I}}^{(2)}=\mathbb{P}\left(\Gamma_{\mathrm{I}}\right) .
\end{aligned}
$$

In addition, we can derive, using Doob's optional sampling theorem, the following two identities:

$$
\begin{aligned}
& \frac{\mathrm{P}_{+, \mathrm{I}}^{(2)}}{\tilde{\mathrm{P}}_{+, \mathrm{I}}^{(2)}}=e^{s_{\mathrm{tot}}^{+} / k_{\mathrm{B}},} \\
& \frac{\mathrm{P}_{-, \mathrm{I}}^{(2)}}{\tilde{\mathrm{P}}_{-, \mathrm{I}}^{(2)}}=e^{-s_{\mathrm{tot}}^{-} / k_{\mathrm{B}}} .
\end{aligned}
$$

For instance, Eq. (D8) follows immediately from Eqs. (39)-(44), if we replace $\Phi_{+}$by $\Phi_{+, \mathrm{I}}$. Note that in Eq. (40), we can still apply Doob's optional sampling theorem since the set $\Phi_{+, \mathrm{I}}$ belongs to the $\sigma$-algebra $\mathcal{F}\left(T^{(2)}\right)$, i.e., $\Phi_{+, \mathrm{I}} \in \mathcal{F}\left(T^{(2)}\right)$, with $T^{(2)}$ the two-boundary firstpassage time of entropy production with thresholds $s_{\text {tot }}^{+}$ and $s_{\text {tot }}^{-}$. From Eqs. (D4)-(D9), we find the following explicit expressions for the passage probabilities $\mathrm{P}_{+, \mathrm{I}}^{(2)}$ and $P_{+, I}^{(2)}$ :

$$
\mathrm{P}_{+, \mathrm{I}}^{(2)}=\mathbb{P}\left(\Gamma_{\mathrm{I}}\right) \frac{e^{s_{\text {tot }}^{-} / k_{\mathrm{B}}}-1}{e^{s_{\mathrm{tot}}^{-} / k_{\mathrm{B}}}-e^{-s_{\text {tot }}^{+} / k_{\mathrm{B}}}}
$$




$$
\mathrm{P}_{-, \mathrm{I}}^{(2)}=\mathbb{P}\left(\Gamma_{\mathrm{I}}\right) \frac{1-e^{-s_{\mathrm{tot}}^{+} / k_{\mathrm{B}}}}{e^{s_{\mathrm{tot}}^{-} / k_{\mathrm{B}}}-e^{-s_{\mathrm{tot}}^{+} / k_{\mathrm{B}}}}
$$

The conditional entropy-production passage probabilities, for trajectories that are initially in the macrostate I, are

$$
\begin{aligned}
& \frac{\mathrm{P}_{+, \mathrm{I}}^{(2)}}{\mathbb{P}\left(\Gamma_{\mathrm{I}}\right)}=\frac{e^{s_{\mathrm{tot}}^{-} / k_{\mathrm{B}}}-1}{e^{s_{\mathrm{tot}}^{-} / k_{\mathrm{B}}}-e^{-s_{\mathrm{tot}}^{+} / k_{\mathrm{B}}}}, \\
& \frac{\mathrm{P}_{-, \mathrm{I}}^{(2)}}{\mathbb{P}\left(\Gamma_{\mathrm{I}}\right)}=\frac{1-e^{-s_{\mathrm{tot}}^{+} / k_{\mathrm{B}}}}{e^{s_{\mathrm{tot}}^{-} / k_{\mathrm{B}}}-e^{-s_{\mathrm{tot}}^{+} / k_{\mathrm{B}}}} .
\end{aligned}
$$

The entropy-production passage probabilities conditioned on the initial state are thus the same as the unconditioned entropy-production passage probabilities, given by Eqs. (45) and (46).

\section{APPENDIX E: STOPPING-TIME FLUCTUATION THEOREMS FOR ENTROPY PRODUCTION}

In this appendix, we derive fluctuation relations for stopping times of entropy production. In the first subsection, we derive the fluctuation relation for entropy stopping times, given by Eq. (64); in the second subsection, we apply this fluctuation relation to first-passage times. In the third subsection, we derive a cumulative fluctuation relation for entropy stopping times of trajectories starting in a macrostate I. In the last subsection, we use this generalized cumulative fluctuation relation to derive the fluctuation relation for waiting times of stochastic processes, Eq. (73).

\section{Fluctuation theorem for entropy stopping times}

The cumulative fluctuation theorem for entropy stopping times, given by Eq. (60) in Sec. VI, follows from the following identities:

$$
\begin{aligned}
& \frac{\mathbb{P}\left(\Theta_{T_{+}}\left(\Phi_{T_{+} \leq t}\right)\right)}{\mathbb{P}\left(\Phi_{T_{+} \leq t}\right)} \\
& =\left.\frac{\int_{\omega \in \Theta_{T_{+}}}\left(\Phi_{T_{+} \leq t}\right)}{\left.\int_{\omega \in \Phi_{T_{+} \leq t}} \mathrm{~d} \mathbb{P}\right|_{\mathcal{F}(t)}}\right|_{\mathcal{F}(t)} \\
& =\frac{\left.\int_{\omega \in \Phi_{T_{+} \leq t}} \mathrm{~d}\left(\mathbb{P} \circ \Theta_{T_{+}}\right)\right|_{\mathcal{F}(t)}}{\left.\int_{\omega \in \Phi_{T_{+} \leq t}} \mathrm{~d} \mathbb{P}\right|_{\mathcal{F}(t)}} \\
& =\frac{\left.\int_{\omega \in \Phi_{T_{+} \leq t}} \mathrm{~d}\left(\mathbb{P} \circ \mathrm{T}_{T_{+}} \circ \Theta\right)\right|_{\mathcal{F}(t)}}{\left.\int_{\omega \in \Phi_{T_{+} \leq t}} \mathrm{~d} \mathbb{P}\right|_{\mathcal{F}(t)}} \\
& =\frac{\left.\int_{\omega \in \Phi_{T_{+} \leq t}} \mathrm{~d}(\mathbb{P} \circ \Theta)\right|_{\mathcal{F}(t)}}{\left.\int_{\omega \in \Phi_{T_{+} \leq t}} \mathrm{~d} \mathbb{P}\right|_{\mathcal{F}(t)}}
\end{aligned}
$$

$$
\begin{aligned}
& =\frac{\left.\int_{\omega \in \Phi_{T_{+} \leq t}} e^{-S_{\mathrm{tot}}(t ; \omega) / k_{\mathrm{B}}} \mathrm{d} \mathbb{P}\right|_{\mathcal{F}(t)}}{\left.\int_{\omega \in \Phi_{T_{+} \leq t}} \mathrm{~d} \mathbb{P}\right|_{\mathcal{F}(t)}} \\
& =\frac{\left.\int_{\omega \in \Phi_{T_{+} \leq t}} e^{-S_{\mathrm{tot}}\left(T_{+} ; \omega\right) / k_{\mathrm{B}}} \mathrm{d} \mathbb{P}\right|_{\mathcal{F}(t)}}{\left.\int_{\omega \in \Phi_{T_{+} \leq t}} \mathrm{~d} \mathbb{P}\right|_{\mathcal{F}(t)}} \\
& =e^{-s_{\mathrm{tot}} / k_{\mathrm{B}}} \frac{\left.\int_{\omega \in \Phi_{T_{+} \leq t}} \mathrm{~d} \mathbb{P}\right|_{\mathcal{F}(t)}}{\left.\int_{\omega \in \Phi_{T_{+} \leq t}} \mathrm{~d} \mathbb{P}\right|_{\mathcal{F}(t)}} \\
& =e^{-S_{\mathrm{tot}} / k_{\mathrm{B}}}
\end{aligned}
$$

Here, we have $0 \leq t<+\infty$. In Eq. (E2), we have written the measures of the $\mathcal{F}(t)$-measurable sets $\Theta_{T_{+}}\left(\Phi_{T_{+} \leq t}\right)$ and $\Phi_{T_{+} \leq t}$ in terms of integrals over these sets with respect to the probability space $\left(\Omega, \mathcal{F}(t),\left.\mathbb{P}\right|_{\mathcal{F}(t)}\right)$. In Eq. (E3), we have transformed the variables of the integral in the numerator with the change-of-variables formula, which we have applied using the measurable morphism $\Theta_{T_{+}}$. The change-of-variables formula relates two integrals under a change of variables, viz.,

$\int_{\omega \in \Phi} X(\omega) \mathrm{d} \mathbb{P}=\int_{\omega \in \phi(\Phi)}\left(X \circ \phi^{-1}\right)(\omega) \mathrm{d}\left(\mathbb{P} \circ \phi^{-1}\right)$,

with $X$ a random variable, measurable in a probability space $(\Omega, \mathcal{F}, \mathbb{P}) ; \Phi$ a measurable set in this probability space, i.e., $\Phi \in \mathcal{F}$; and $\phi: \mathcal{F} \rightarrow \mathcal{F}^{\prime}$ a measurable morphism from one probability space $(\Omega, \mathcal{F}, \mathbb{P})$ to another probability space $\left(\Omega^{\prime}, \mathcal{F}^{\prime}, \mathbb{P} \circ \phi\right)$, with the property that $\phi^{-1}(\Xi) \in \mathcal{F}$ for each $\Xi \in \mathcal{F}^{\prime}$ (see exercise 1.4.38 in Ref. [56]). In Eq. (E4), we have used the definition of the composition $\Theta_{T_{+}}=\mathrm{T}_{T_{+}} \circ \Theta$. In Eq. (E5), we have used that $\mathbb{P}$ is a stationary measure, and thus $\mathbb{P}=\mathbb{P} \circ T_{T_{+}}$. In Eq. (E6), we have used the Radon-Nikodým theorem, given by Eq. (17), in order to change the integral with respect to the measure $\mathbb{P} \circ \Theta$ into an integral with respect to the measure $\mathbb{P}$; subsequently, we have used our measure-theoretic definition of entropy production, given by Eq. (18), to write the Radon-Nikodým derivative in terms of entropy production $S_{\text {tot }}(t)$. Since $e^{-S_{\text {tot }}(t) / k_{\mathrm{B}}}$ is a uniformly integrable martingale with respect to $\mathbb{P}$, in Eq. (E7) we have used Doob's optional sampling theorem Eq. (A8). In order to apply this theorem, we additionally need $e^{-S_{\text {tot }}(t) / k_{\mathrm{B}}}$ to be right continuous. In Eq. (E8), we have used the fact that $T_{+}(\omega)$ is an $s_{\text {tot }}$-stopping time such that $s_{\text {tot }}=S_{\text {tot }}\left(\omega ; T_{+}(\omega)\right)$. Finally, in Eq. (E9), we have used that the measure of the set $\Phi_{T_{+} \leq t}$ is larger than zero, and that $a / a=1$ for $a \neq 0$.

The fluctuation theorem Eq. (60) can be written as a ratio between the cumulative distribution of the $s_{\text {tot }}$-stopping time $T_{+}$and the cumulative distribution of a conjugate $\left(-s_{\text {tot }}\right)$-stopping time $T_{-}$. We define the $\left(-s_{\text {tot }}\right)$-stopping time $T_{-}$associated with the stopping time $T_{+}$through the relation 


$$
T_{-}\left(\Theta_{T_{+}(\omega)}(\omega)\right)=T_{+}(\omega) .
$$

We thus have

$$
\Phi_{T_{-} \leq t}=\Theta_{T_{+}}\left(\Phi_{T_{+} \leq t}\right),
$$

and the cumulative fluctuation relation, given by Eq. (60), reads for $0 \leq t<+\infty$

$$
\frac{\mathbb{P}\left(\Phi_{T_{-} \leq t}\right)}{\mathbb{P}\left(\Phi_{T_{+} \leq t}\right)}=e^{s_{\mathrm{tot}} / k_{\mathrm{B}}}
$$

Since the probability-density functions of $T_{+}$and $T_{-}$are, respectively, given by

$$
\begin{aligned}
& p_{T_{+}}(t)=\frac{\mathrm{d}}{\mathrm{d} t} \mathbb{P}\left(\Phi_{T_{+} \leq t}\right), \\
& p_{T_{-}}(t)=\frac{\mathrm{d}}{\mathrm{d} t} \mathbb{P}\left(\Phi_{T_{-} \leq t}\right),
\end{aligned}
$$

we find the following fluctuation theorem for the distributions of entropy stopping times:

$$
\frac{p_{T_{+}}(t)}{p_{T_{-}}(t)}=e^{s_{\mathrm{tot}} / k_{\mathrm{B}}}
$$

which is precisely the stopping-time fluctuation theorem Eq. (64).

\section{First-passage time fluctuation theorems for entropy production}

We apply the fluctuation theorem, given by Eq. (E16), to first-passage times of entropy production. We consider here first-passage times with one absorbing boundary $T_{ \pm}^{(1)}$ and first-passage times with two absorbing boundaries $T_{ \pm}^{(2)}$ (see Sec. VI A).

The first-passage time $T_{ \pm}^{(1)}$ denotes the time when the process $S_{\text {tot }}(\omega ; t)$ first passes the threshold $\pm s_{\text {tot }}$. If $S_{\text {tot }}(\omega ; t)$ never passes the threshold $\pm s_{\text {tot }}$, then $T_{ \pm}^{(1)}=+\infty$.

The first-passage time $T_{+}^{(2)}$ denotes the time when the process $S_{\text {tot }}(\omega ; t)$ first passes the threshold $s_{\text {tot }}$, given that it has not reached $-s_{\text {tot }}$ before:

$$
T_{+}^{(2)}= \begin{cases}T_{+}^{(1)} & T_{+}^{(1)}<T_{-}^{(1)}, \\ +\infty & T_{+}^{(1)}>T_{-}^{(1)} .\end{cases}
$$

Analogously, we define $T_{-}^{(2)}$ :

$$
T_{-}^{(2)}= \begin{cases}+\infty & T_{+}^{(1)}<T_{-}^{(1)}, \\ T_{-}^{(1)} & T_{+}^{(1)}>T_{-}^{(1)} .\end{cases}
$$

Since entropy production is a process of odd parity with respect to the time-reversal map $\Theta_{t}$ [see Eq. (B1)], we have the relations

$$
\Theta_{T_{+}^{(1)}}\left(\Phi_{T_{+}^{(1)} \leq t}\right)=\Phi_{T_{-}^{(1)} \leq t}
$$

and

$$
\Theta_{T_{+}^{(2)}}\left(\Phi_{T_{+}^{(2)} \leq t}\right)=\Phi_{T_{-}^{(2)} \leq t} .
$$

Using Eqs. (E12), (E13), (E19), and (E20), we thus find the first-passage-time fluctuation relations:

$$
\frac{p_{T_{+}^{(1)}}(t)}{p_{T_{-}^{(1)}}(t)}=e^{s_{\mathrm{tot}} / k_{\mathrm{B}}}
$$

and

$$
\frac{p_{T_{+}^{(2)}}(t)}{p_{T_{-}^{(2)}}(t)}=e^{s_{\mathrm{tot}} / k_{\mathrm{B}}} .
$$

\section{Generalized cumulative fluctuation theorem for stopping times of entropy production}

We define macrostates as subsets I [II] of the phase space of configurations $\left\{\boldsymbol{q}, \boldsymbol{q}^{*}\right\}$. We also consider the subsets $\tilde{\mathrm{I}}[\tilde{\mathrm{II}}]$ of the corresponding time-reversed states $\tilde{\mathrm{I}}=\left\{\left(\boldsymbol{q}, \boldsymbol{q}^{*}\right):\left(\boldsymbol{q},-\boldsymbol{q}^{*}\right) \in \mathrm{I}\right\} \quad\left[\tilde{\mathrm{II}}=\left\{\left(\boldsymbol{q}, \boldsymbol{q}^{*}\right):\left(\boldsymbol{q},-\boldsymbol{q}^{*}\right) \in \mathrm{II}\right\}\right]$. With each macrostate I, we associate a set of trajectories $\Gamma_{\mathrm{I}}$ :

$$
\Gamma_{\mathrm{I}}=\{\omega \in \Omega: \omega(0) \in \mathrm{I}\} .
$$

Note that Doob's optional sampling theorem, given by Eq. (A8), applies to the set $\Phi_{T_{+} \leq t} \cap \Gamma_{\mathrm{I}}$ since $\Phi_{T_{+} \leq t} \cap \Gamma_{\mathrm{I}} \in \mathcal{F}\left(T_{+}\right)$. We can therefore follow the steps of Eqs. (E1)-(E8) in order to find the following generalized cumulative fluctuation relation:

$$
\frac{\mathbb{P}\left(\Theta_{T_{+}}\left(\Phi_{T_{+} \leq t} \cap \Gamma_{\mathrm{I}}\right)\right)}{\mathbb{P}\left(\Phi_{T_{+} \leq t} \cap \Gamma_{\mathrm{I}}\right)}=e^{-s_{\text {tot }} / k_{\mathrm{B}}} .
$$

\section{Fluctuation relations for waiting times}

Waiting times $T^{\mathrm{I} \rightarrow \mathrm{II}}$ denote the time a process takes to travel between two macrostates I and II (the time it takes for the system to change its macrostate from I to II). Here, we derive a fluctuation theorem for waiting times along trajectories that produce a given amount of entropy production. The entropy waiting time $T_{+}^{\mathrm{I} \rightarrow \mathrm{II}}$ denotes the time a process needs to travel from the macrostate I to the macrostate II while producing a positive entropy $s_{\text {tot }}$, and given that the process has not returned to the macrostate I before. Analogously, the entropy waiting time $T_{-}^{\tilde{I} \rightarrow \tilde{I}}$ denotes the time a process needs to travel from the macrostate II to the macrostate I while producing a negative entropy $-s_{\text {tot }}$, and given that the process has not returned to the macrostate II before. 
In order to define waiting times, we first define stopping times $T^{\mathrm{I}}$ and $T^{\mathrm{II}}$ that denote the time when a process reaches a given macrostate I or II, respectively:

$$
\begin{gathered}
T^{\mathrm{I}}(\omega)=\inf \{t>0 \cup\{+\infty\}: \omega(t) \in \mathrm{I}\}, \\
T^{\mathrm{II}}(\omega)=\inf \{t>0 \cup\{+\infty\}: \omega(t) \in \mathrm{II}\} .
\end{gathered}
$$

The waiting time $T^{\mathrm{I} \rightarrow \mathrm{II}}$ denotes the time a process takes to travel from I to II:

$$
T^{\mathrm{I} \rightarrow \mathrm{II}}(\omega)= \begin{cases}T^{\mathrm{II}}(\omega) & T^{\mathrm{II}}(\omega)<T^{\mathrm{I}}(\omega), \\ +\infty & T^{\mathrm{II}}(\omega)>T^{\mathrm{I}}(\omega),\end{cases}
$$

for all trajectories $\omega$ for which $\omega(0) \in \mathrm{I}$, and the associated $s_{\text {tot }}$-waiting time $T_{+}^{\mathrm{I} \rightarrow \mathrm{II}}$ is defined as

$T_{+}^{\mathrm{I} \rightarrow \mathrm{II}}(\omega)=\left\{\begin{array}{cc}T^{\mathrm{I} \rightarrow \mathrm{II}}(\omega) & S_{\text {tot }}\left(T^{\mathrm{I} \rightarrow \mathrm{II}}(\omega) ; \omega\right)=s_{\text {tot }}, \\ +\infty & S_{\text {tot }}\left(T^{\mathrm{I} \rightarrow \mathrm{II}}(\omega) ; \omega\right) \neq s_{\text {tot }},\end{array}\right.$

for all trajectories $\omega$ for which $\omega(0) \in \mathrm{I}$. We also define the $\left(-s_{\text {tot }}\right)$-stopping time $T_{-}^{\tilde{I} \rightarrow \tilde{I}}$, denoting the time it takes for a process to travel from the macrostate II to the macrostate I, while producing a negative entropy $s_{\mathrm{tot}}$ :

$T_{-}^{\tilde{\mathrm{I}} \rightarrow \tilde{\mathrm{I}}}(\omega)= \begin{cases}T^{\tilde{\mathrm{I}} \rightarrow \mathrm{I}}(\omega) & S_{\text {tot }}\left(T^{\tilde{\mathrm{I}} \rightarrow \tilde{\mathrm{I}}}(\omega) ; \omega\right)=-s_{\text {tot }}, \\ +\infty & S_{\text {tot }}\left(T^{\tilde{\mathrm{I}} \rightarrow \tilde{\mathrm{I}}}(\omega) ; \omega\right) \neq-s_{\text {tot }},\end{cases}$

for all trajectories $\omega$ for which $\omega(0) \in \tilde{I I}$

We apply the generalized cumulative fluctuation theorem, given by Eq. (E24), to the stopping time $T_{+}^{\mathrm{I} \rightarrow \mathrm{II}}$ and the set $\Gamma_{\mathrm{I}}$ defined in (E23), and find for $0 \leq t<+\infty$

$$
\frac{\mathbb{P}\left(\Phi_{T_{+}^{I \rightarrow \mathrm{I}} \leq t} \cap \Gamma_{\mathrm{I}}\right)}{\mathbb{P}\left(\Theta_{T_{+}^{\mathrm{I} \rightarrow \mathrm{II}}}\left(\Phi_{T_{+}^{\mathrm{I} I \mathrm{II}} \leq t} \cap \Gamma_{\mathrm{I}}\right)\right)}=e^{s_{\mathrm{tot}} / k_{\mathrm{B}}} .
$$

Notice that the waiting time $T_{+}^{\mathrm{I} \rightarrow \mathrm{II}}(\omega)$ is defined on all trajectories $\omega \in \Omega$, but we are interested in those trajectories for which $\omega(0) \in \mathrm{I}$, and we have thus set $\omega \in \Gamma_{\mathrm{I}}$. Since

$$
\Theta_{T_{+}^{\mathrm{I} \rightarrow \mathrm{II}}}\left(\Phi_{T_{+}^{\mathrm{I} \rightarrow \mathrm{I}} \leq t} \cap \Gamma_{\mathrm{I}}\right)=\Phi_{T_{-}^{\tilde{\mathrm{I}} \rightarrow \tilde{\mathrm{I}}} \leq t} \cap \Gamma_{\tilde{\tilde{I}}},
$$

we have

$$
\frac{\mathbb{P}\left(\Phi_{T_{+}^{\mathrm{I}}+\mathbb{I} \leq t} \cap \Gamma_{\mathrm{I}}\right)}{\mathbb{P}\left(\Phi_{T_{-}^{\tilde{I} \sim \tilde{I}} \leq t} \cap \Gamma_{\tilde{\mathrm{I}}}\right)}=e^{s_{\mathrm{tot}} / k_{\mathrm{B}}} .
$$

We write the probability densities of the entropy waiting times $T_{+}^{\mathrm{I} \rightarrow \mathrm{II}}$ and $T_{-}^{\tilde{\mathrm{I}} \rightarrow \mathrm{I}}$ in terms of the measures on the lefthand side of the previous equation:

$$
\begin{aligned}
& p_{T_{+}^{\mathrm{I} \rightarrow \mathrm{II}}}(t)=\frac{1}{\mathbb{P}\left(\Gamma_{\mathrm{I}}\right)} \frac{\mathrm{d}}{\mathrm{d} t} \mathbb{P}\left(\Phi_{T_{+}^{\mathrm{I} \rightarrow \mathrm{I}} \leq t} \cap \Gamma_{\mathrm{I}}\right), \\
& p_{T_{-}^{\tilde{\mathrm{I}} \tilde{\mathrm{I}}}}(t)=\frac{1}{\mathbb{P}\left[\Gamma_{\tilde{\mathrm{I}}}\right]} \frac{\mathrm{d}}{\mathrm{d} t} \mathbb{P}\left(\Phi_{T_{-}^{\tilde{\mathrm{I}} \rightarrow \tilde{\mathrm{I}}} \leq t} \cap \Gamma_{\tilde{\mathrm{I}}}\right),
\end{aligned}
$$

which holds for $0 \leq t<+\infty$. We thus find the following fluctuation theorem for waiting times between macrostates:

$$
\frac{p_{T_{+}^{I \rightarrow I I}}(t)}{p_{T_{-}^{\tilde{I}} \rightarrow \tilde{I}}(t)}=e^{s_{\mathrm{tot}} / k_{\mathrm{B}}+\log _{\frac{\mathbb{P}}{\mathrm{P}\left(\Gamma_{\tilde{I}} \tilde{I}\right)}}}
$$

If we set $\Gamma_{\mathrm{I}}=\Gamma_{\mathrm{II}}=\Omega$, Eq. (E35) is equal to the entropy stopping-time fluctuation theorem, given by Eq. (E16).

The quantity in the exponential of the right-hand side of Eq. (E35) has, in general, no particular meaning. For macrostates defined by variables of even parity, i.e., $\tilde{\mathrm{I}}=\mathrm{I}$ and $\tilde{\mathrm{II}}=\mathrm{II}$, we have the relation $\Gamma_{\tilde{\mathrm{II}}}=\Gamma_{\mathrm{II}}$. Then, we recognize in Eq. (E35) the system entropy change:

$$
\Delta s_{\mathrm{sys}}=-k_{\mathrm{B}} \log \frac{\mathbb{P}\left(\Gamma_{\mathrm{II}}\right)}{\mathbb{P}\left(\Gamma_{\mathrm{I}}\right)},
$$

between the two macrostates I and II.

A particular important example of the waiting-time fluctuation theorem, given by Eq. (E35), is for macrostates I and II, which correspond to one single point in phase space, i.e., I $=\left\{\boldsymbol{q}_{\mathrm{I}}\right\}$ and II $=\left\{\boldsymbol{q}_{\mathrm{II}}\right\}$. Then, we find the relation

$$
\frac{p_{T_{+}^{I \rightarrow \mathrm{II}}}(t)}{p_{T_{-}^{\mathrm{II}} \rightarrow \mathrm{I}}(t)}=e^{s_{\mathrm{env}} / k_{\mathrm{B}}},
$$

with $s_{\text {env }}=s_{\text {tot }}-\Delta s_{\text {sys }}$ the change in the environment entropy and $\Delta s_{\text {sys }}$ the system-entropy change, which here is given by

$$
\Delta s_{\mathrm{sys}}=-k_{\mathrm{B}} \log \frac{p_{\mathrm{ss}}\left(\boldsymbol{q}_{\mathrm{II}}\right)}{p_{\mathrm{ss}}\left(\boldsymbol{q}_{\mathrm{I}}\right)} .
$$

\section{APPENDIX F: INFIMUM STATISTICS FOR THE DRIFT-DIFFUSION PROCESS}

In this appendix, we derive explicit expressions for the distribution and the mean of the infimum of entropy production in the drift-diffusion process. Since in a drift-diffusion process entropy production is $S_{\text {tot }}(t) / k_{\mathrm{B}}=(v / D)[X(t)-X(0)]$, the infimum statistics of entropy production follow from the infimum statistics of the position coordinate $X(t)$. Therefore, we first derive the extreme value statistics of $X(t)$, and subsequently we derive the infimum statistics of entropy production.

\section{Cumulative distribution of the infimum of entropy production}

The cumulative distribution $\operatorname{Pr}(\sup X(t) \leq L)$ of the supremum $\sup X(t) \equiv \sup _{\tau \in[0, t]} X(\tau) \quad$ of a stochastic 
process, over a time interval $[0, t]$, equals the survival probability $Q_{X}(L, t)$ of the process, in the interval $(-\infty, L)$ at time $t[104,121]$ :

$\operatorname{Pr}(\sup X(t) \leq L)=\operatorname{Pr}(X(s) \leq L ; s \leq t)=Q_{X}(L, t)$,

with $L>0$. For a general stochastic process, the survival probability in an interval can be calculated from the distribution $p_{T}(\tau ; L)$ of first-passage times to reach an absorbing boundary located at $L$ :

$$
Q_{X}(L, t)=1-\int_{0}^{t} \mathrm{~d} \tau p_{T}(\tau ; L)
$$

We use this relation between survival probabilities and firstpassage-time distributions to determine the cumulative distribution of extreme values of $X(t)$.

The infimum of a drift-diffusion process with positive drift is equal to the supremum of a drift-diffusion process with the same but negative drift. We therefore consider the following two conjugate drift-diffusion processes:

(1) $X_{+}(t)$ with velocity $v$, diffusion $D$, and initial condition $X_{+}(0)=0$,

(2) $X_{-}(t)$ with velocity $-v$ and diffusion $D$, and initial condition $X_{-}(0)=0$,

and $v>0$ in both processes. The dynamics of both processes follows from Eq. (76) for $V(x)=0$ and for, respectively, a positive and negative velocity. The infimum value of $X_{+}(t)$ is equal to minus the supremum of the conjugate process $X_{-}(t)$. In the following, we derive analytical expressions for the statistics of the supremum of $X_{-}(t)$ and use these to obtain the statistics of the infimum of $X_{+}(t)$.

The survival probability of $X_{-}(t)$ can be obtained from the distribution $p_{T}$ of first-passage times to first reach the threshold $L$; see Eq. (F2). This first-passage-time distribution reads

$$
p_{T}(t ; L)=\frac{L}{\sqrt{4 \pi D t^{3}}} e^{-(L+v t)^{2} / 4 D t} .
$$

Substituting Eq. (F3) in Eq. (F2) results in the following expression for the survival probability of $X_{-}$:

$$
\begin{aligned}
Q_{X_{-}}(L, t)= & 1-\frac{1}{2}\left[\operatorname{erfc}\left(\frac{L+v t}{\sqrt{4 D t}}\right)\right. \\
& \left.+e^{-v L / D} \operatorname{erfc}\left(\frac{L-v t}{\sqrt{4 D t}}\right)\right],
\end{aligned}
$$

where erfc is the complementary error function. Equation (F4) yields the cumulative density function of the supremum of $X_{-}$, as follows from Eq. (F1).

From the relation between the conjugate processes, we relate the cumulative distribution of the infimum of $X_{+}$over the interval $[0, t], \inf X_{+}(t)$, to the survival probability of $X_{-}(t)$ :

$$
\begin{aligned}
& \operatorname{Pr}\left(-\inf X_{+}(t) \leq L\right) \\
& =\operatorname{Pr}\left(\inf X_{+}(t) \geq-L\right) \\
& =\operatorname{Pr}\left(\sup X_{-}(t) \leq L\right) \\
& =Q_{X_{-}}(L, t) .
\end{aligned}
$$

Using Eq. (F4) and the property $\operatorname{erfc}(x)+\operatorname{erfc}(-x)=2$, we obtain an analytical expression for the cumulative distribution of the infimum of the position of a driftdiffusion process with positive velocity:

$$
\begin{aligned}
\operatorname{Pr}\left(-\inf X_{+}(t) \leq L\right)= & \frac{1}{2}\left[\operatorname{erfc}\left(\frac{-L-v t}{\sqrt{4 D t}}\right)\right. \\
& \left.-e^{-v L / D} \operatorname{erfc}\left(\frac{L-v t}{\sqrt{4 D t}}\right)\right]
\end{aligned}
$$

Finally, for the stochastic process $S_{\text {tot }}(t) / k_{\mathrm{B}}=$ $(v / D) X_{+}(t)$, the infimum distribution can be obtained by replacing $v$ and $D$ in Eq. (F6) by the effective values for the process $S_{\mathrm{tot}}(t) / k_{\mathrm{B}}$, given by $v_{\mathrm{eff}}=v^{2} / D$ and $D_{\text {eff }}=v^{2} / D$. Defining $\quad \bar{s}(t)=\left\langle S_{\text {tot }}(t)\right\rangle / k_{\mathrm{B}}=\left(v^{2} / D\right) t$, we obtain

$$
\begin{aligned}
\operatorname{Pr}\left(-\frac{S_{\text {inf }}(t)}{k} \leq s\right)= & \frac{1}{2}\left[\operatorname{erfc}\left(\frac{-s-\bar{s}(t)}{2 \sqrt{\bar{s}(t)}}\right)\right. \\
& \left.-e^{-s} \operatorname{erfc}\left(\frac{s-\bar{s}(t)}{2 \sqrt{\bar{s}(t)}}\right)\right],
\end{aligned}
$$

which is equal to Eq. (81).

\section{Mean infimum of entropy production}

We first determine the distribution of the infimum of $X_{+}$and then compute its mean value. Note that the infimum of $X_{+}$equals minus the supremum of the conjugate process $X_{-}$:

$$
\left\langle\inf X_{+}(t)\right\rangle=-\left\langle\sup X_{-}(t)\right\rangle .
$$

The cumulative distribution of the supremum of $X_{-}$is given by

$$
\begin{aligned}
\operatorname{Pr} & \left(\sup X_{-}(t) \leq L\right) \\
& =Q_{X_{-}}(L, t) \\
& =\frac{1}{2}\left[\operatorname{erfc}\left(\frac{-L-v t}{\sqrt{4 D t}}\right)-e^{-v L / D} \operatorname{erfc}\left(\frac{L-v t}{\sqrt{4 D t}}\right)\right],
\end{aligned}
$$

where we have used Eq. (F4) and the property $\operatorname{erfc}(x)+\operatorname{erfc}(-x)=2$. The distribution of the supremum of $X_{-}$can be found by deriving Eq. (F9) with respect to $L$, which yields 


$$
\begin{aligned}
\operatorname{Pr}\left(\sup X_{-}(t)=L\right)= & \frac{1}{\sqrt{\pi D t}} e^{-v^{2} t / 4 D} \\
& +\frac{v}{2 D} e^{-v L / D} \operatorname{erfc}\left(\frac{v \sqrt{t}}{2 \sqrt{D}}\right) .
\end{aligned}
$$

The mean of the supremum of $X_{-}$is the integral

$$
\left\langle\sup X_{-}(t)\right\rangle=\int_{0}^{\infty} \mathrm{d} L \operatorname{Pr}\left(\sup X_{-}(t)=L\right) L,
$$

which, after some algebra, yields

$$
\begin{aligned}
\left\langle\sup X_{-}(t)\right\rangle= & \frac{D}{v} \operatorname{erf}\left(\frac{v \sqrt{t}}{2 \sqrt{D}}\right)-\frac{v t}{2} \operatorname{erfc}\left(\frac{v \sqrt{t}}{2 \sqrt{D}}\right) \\
& +\sqrt{\frac{D t}{\pi}} e^{-v^{2} t / 4 D} .
\end{aligned}
$$

From Eqs. (F8) and (F12), we find an exact expression for the mean infimum of a drift-diffusion process with positive velocity:

$$
\begin{aligned}
\left\langle\inf X_{+}(t)\right\rangle= & -\frac{D}{v} \operatorname{erf}\left(\frac{v \sqrt{t}}{2 \sqrt{D}}\right)+\frac{v t}{2} \operatorname{erfc}\left(\frac{v \sqrt{t}}{2 \sqrt{D}}\right) \\
& -\sqrt{\frac{D t}{\pi}} e^{-v^{2} t / 4 D} .
\end{aligned}
$$

Using $\quad \bar{s}(t)=\left\langle S_{\text {tot }}(t)\right\rangle / k_{\mathrm{B}}=\left(v^{2} / D\right) t \quad$ and $\quad S_{\mathrm{tot}}(t)=$ $(v / D) X_{+}(t)$, we obtain, from the expression (F13), an analytical expression for the mean infimum of entropy production in a drift-diffusion process at time $t$ :

$$
\begin{aligned}
\left\langle\frac{S_{\mathrm{inf}}(t)}{k_{\mathrm{B}}}\right\rangle= & -\operatorname{erf}\left(\frac{\sqrt{\bar{s}(t)}}{2}\right)+\frac{\bar{s}(t)}{2} \operatorname{erfc}\left(\frac{\sqrt{\bar{s}(t)}}{2}\right) \\
& -\sqrt{\frac{\bar{s}(t)}{\pi}} e^{-\bar{s}(t) / 4} .
\end{aligned}
$$

The above result is Eq. (82) in the main text.

\section{APPENDIX G: PASSAGE PROBABILITIES AND FIRST-PASSAGE TIMES FOR THE DRIFT-DIFFUSION PROCESS}

We determine the passage probabilities and the firstpassage-time statistics of entropy production for a drifted Brownian particle with diffusion coefficient $D$ and drift velocity $v$, as described by the Langevin Eq. (76) [with $V(x)=0]$. Since in a drift-diffusion process entropy production is $S_{\mathrm{tot}}(t) / k_{\mathrm{B}}=(v / D)[X(t)-X(0)]$, the passage probabilities and the first-passage-time statistics of entropy production follow from the corresponding statistics of $X(t)$.

\section{First-passage-time statistics for one absorbing boundary or two symmetric absorbing boundaries}

The first-passage-time distribution for $X$ to pass the threshold $L>0$ at time $T=t$ for the first time, starting from the initial condition $X(0)=0$, is given by Wald's distribution $[122,123]$

$$
p_{T}(t ; L)=\frac{|L|}{\sqrt{4 \pi D t^{3}}} e^{-(L-v t)^{2} / 4 D t} .
$$

Equation (G1) implies

$$
\frac{p_{T}(t ; L)}{p_{T}(t ;-L)}=e^{v L / D}
$$

Note that the argument of the exponential is equal to the Péclet number $\mathrm{Pe}=v L / D$.

The distribution of entropy first-passage times $T_{+}^{(1)}$ with one absorbing boundary located at $s_{\text {tot }}$ is equal to the firstpassage-time distribution for the position of the particle, given by Eq. (G1), with one absorbing boundary located at $L=s_{\text {tot }} D /\left(v k_{\mathrm{B}}\right)$. Equation (G2) implies thus a fluctuation theorem for first-passage times of entropy production, consistent with the fluctuation theorem for stopping times given by Eq. (8). An analogous relation to Eq. (G2) holds for two-boundary first-passage times $T_{+}^{(2)}$ for entropy production in the drift-diffusion process and can be derived using the results in Sec. 2.2.2.2 in Ref. [122] (see also Ref. [47]).

\section{Comparing first-passage-time statistics for two asymmetric absorbing boundaries}

We consider the drift-diffusion process with two absorbing boundaries located at $L_{+} \geq 0$ and $-L_{-} \leq 0$, and with the initial position $X(0)=0$. The passage probabilities $\mathrm{P}_{+}^{(2)}$ and $\mathrm{P}_{-}^{(2)}$, to first reach $L_{+}$and $-L_{-}$, respectively, are [122]

$$
\begin{aligned}
& \mathbf{P}_{+}^{(2)}=\frac{e^{v L_{-} / D}-1}{e^{v L_{-} / D}-e^{-v L_{+} / D}}, \\
& \mathbf{P}_{-}^{(2)}=\frac{1-e^{-v L_{+} / D}}{e^{v L_{-} / D}-e^{-v L_{+} / D}} .
\end{aligned}
$$

The corresponding entropy-production passage probabilities follow from the expressions (G3) and (G4) using the threshold values $s_{\text {tot }}^{-} / k_{\mathrm{B}}=v L_{-} / D$ and $s_{\text {tot }}^{+} / k_{\mathrm{B}}=v L_{+} / D$. Equations (G3) and (G4) are in correspondence with Eqs. (5) and (6) for passage probabilities of entropy production.

Notably, the first-passage-time fluctuation theorem, given by Eq. (8), does not simply extend to asymmetric boundaries. From the expression (G1) for the first-passagetime distribution of the position with one absorbing boundary, we find 


$$
\frac{p_{T}\left(t ; L_{+}\right)}{p_{T}\left(t ;-L_{-}\right)}=\frac{L_{+}}{L_{-}} e^{v\left(L_{+}-L_{-}\right) /(2 D)} e^{-\left(L_{+}^{2}-L_{-}^{2}\right) /(4 D t)}
$$

Using $s_{\text {tot }}^{-}=v L_{-} / D, s_{\text {tot }}^{+}=v L_{+} / D$, and $\bar{s}=\left(v^{2} / D\right) t$, we find for the ratio of the first-passage time distributions of entropy production

$$
\frac{p_{T_{+}^{(1)}}\left(t ; s_{\text {tot }}^{+}\right)}{p_{T_{-}^{(1)}}\left(t ;-s_{\text {tot }}^{-}\right)}=\frac{s_{\text {tot }}^{+}}{s_{\text {tot }}^{-}} e^{\left(s_{\text {tot }}^{+}+s_{\text {tot }}^{-}\right)\left(1-\frac{s_{\text {tot }}^{+}-s_{\text {tot }}^{-}}{2 k_{\mathrm{B}} \bar{s}(t)}\right) /\left(2 k_{\mathrm{B}}\right)} .
$$

For asymmetric boundaries, the ratio of the first-passagetime distributions is time dependent and converges to a finite value in the limit $t \rightarrow \infty$ [78]. Consequently, for asymmetric boundaries, the mean first-passage time for entropy production to reach the positive threshold $s_{\text {tot }}^{+}$ is different than the mean first-passage time for entropy production to reach the negative threshold $s_{\text {tot }}^{-}$, $\left\langle T_{+}^{(1)}\right\rangle \neq\left\langle T_{-}^{(1)}\right\rangle$. When $s_{\text {tot }}^{+}=s_{\text {tot }}^{-}=s_{\text {tot }}$, we recover the time-independent ratio, $e^{s_{\text {tot }} / k_{\mathrm{B}}}$, in accordance with the first-passage-time fluctuation theorem Eq. (8).

[1] J. C. Maxwell, Taits Thermodynamics, Nature 17, 660 (1878).

[2] G. Bochkov and I. E. Kuzovlev, General Theory of Thermal Fluctuations in Nonlinear Systems, Zh. Eksp. Teor. Fiz. 72, 238 (1977) [J. Exp. Theor. Phys. 45, 125 (1977)].

[3] D. J. Evans, E. Cohen, and G. Morriss, Probability of Second Law Violations in Shearing Steady States, Phys. Rev. Lett. 71, 2401 (1993).

[4] D. J. Evans and D. J. Searles, Equilibrium Microstates which Generate Second Law Violating Steady States, Phys. Rev. E 50, 1645 (1994).

[5] G. Gallavotti and E. Cohen, Dynamical Ensembles in Nonequilibrium Statistical Mechanics, Phys. Rev. Lett. 74, 2694 (1995).

[6] K. Sekimoto, Langevin Equation and Thermodynamics, Prog. Theor. Phys. Suppl. 130, 17 (1998).

[7] G. E. Crooks, Nonequilibrium Measurements of Free Energy Differences for Microscopically Reversible Markovian Systems, J. Stat. Phys. 90, 1481 (1998).

[8] G. E. Crooks, Entropy Production Fluctuation Theorem and the Nonequilibrium Work Relation for Free Energy Differences, Phys. Rev. E 60, 2721 (1999).

[9] J. L. Lebowitz and H. Spohn, A Gallavotti-Cohen-Type Symmetry in the Large Deviation Functional for Stochastic Dynamics, J. Stat. Phys. 95, 333 (1999).

[10] C. Maes, The Fluctuation Theorem as a Gibbs Property, J. Stat. Phys. 95, 367 (1999).

[11] C. Maes and K. Netočný, Time-Reversal and Entropy, J. Stat. Phys. 110, 269 (2003).

[12] P. Gaspard, Time-Reversed Dynamical Entropy and Irreversibility in Markovian Random Processes, J. Stat. Phys. 117, 599 (2004).
[13] D.-Q. Jiang, M. Qian, and M.-P. Qian, Mathematical Theory of Nonequilibrium Steady States: On the Frontier of Probability and Dynamical Systems, No. 1833 (Springer Science \& Business Media, Berlin, 2004).

[14] U. Seifert, Entropy Production Along a Stochastic Trajectory and an Integral Fluctuation Theorem, Phys. Rev. Lett. 95, 040602 (2005).

[15] R. Kawai, J. M. R. Parrondo, and C. V. den Broeck, Dissipation: The Phase-Space Perspective, Phys. Rev. Lett. 98, 080602 (2007).

[16] K. Sekimoto, Stochastic Energetics (Springer, Berlin, 2010), Vol. 799.

[17] R. E. Spinney and I. J. Ford, Entropy Production in Full Phase Space for Continuous Stochastic Dynamics, Phys. Rev. E 85, 051113 (2012).

[18] E. Roldán and J. M. R. Parrondo, Entropy Production and Kullback-Leibler Divergence between Stationary Trajectories of Discrete Systems, Phys. Rev. E 85, 031129 (2012).

[19] Y. Murashita, K. Funo, and M. Ueda, Nonequilibrium Equalities in Absolutely Irreversible Processes, Phys. Rev. E 90, 042110 (2014).

[20] S. Bo and A. Celani, Multiple-Scale Stochastic Processes: Decimation, Averaging and Beyond, Phys. Rep. 670, 1 (2017).

[21] J. Kurchan, Fluctuation Theorem for Stochastic Dynamics, J. Phys. A 31, 3719 (1998).

[22] T. Speck and U. Seifert, The Jarzynski Relation, Fluctuation Theorems, and Stochastic Thermodynamics for Non-Markovian Processes, J. Stat. Mech. (2007) L09002.

[23] R. Chetrite and K. Gawędzki, Fluctuation Relations for Diffusion Processes, Commun. Math. Phys. 282, 469 (2008).

[24] A. Saha, S. Lahiri, and A. M. Jayannavar, Entropy Production Theorems and Some Consequences, Phys. Rev. E 80, 011117 (2009).

[25] C. Aron, G. Biroli, and L. F. Cugliandolo, Symmetries of Generating Functionals of Langevin Processes with Colored Multiplicative Noise, J. Stat. Mech. (2010) P11018.

[26] R. Chetrite and S. Gupta, Two Refreshing Views of Fluctuation Theorems through Kinematics Elements and Exponential Martingale, J. Stat. Phys. 143, 543 (2011).

[27] S. Ciliberto and C. Laroche, An Experimental Test of the Gallavotti-Cohen Fluctuation Theorem, J. Phys. IV 08, Pr6 (1998).

[28] G. Wang, E. M. Sevick, E. Mittag, D. J. Searles, and D. J. Evans, Experimental Demonstration of Violations of the Second Law of Thermodynamics for Small Systems and Short Time Scales, Phys. Rev. Lett. 89, 050601 (2002).

[29] G. Hummer and A. Szabo, Free Energy Surfaces from Single-Molecule Force Spectroscopy, Acc. Chem. Res. 38, 504 (2005).

[30] T. Speck, V. Blickle, C. Bechinger, and U. Seifert, Distribution of Entropy Production for a Colloidal Particle in a Nonequilibrium Steady State, Europhys. Lett. 79, 30002 (2007).

[31] J. R. Gomez-Solano, L. Bellon, A. Petrosyan, and S. Ciliberto, Steady-State Fluctuation Relations for Systems 
Driven by an External Random Force, Europhys. Lett. 89, 60003 (2010).

[32] N. Garnier and S. Ciliberto, Nonequilibrium Fluctuations in a Resistor, Phys. Rev. E 71, 060101 (2005).

[33] K. Feitosa and N. Menon, Fluidized Granular Medium as an Instance of the Fluctuation Theorem, Phys. Rev. Lett. 92, 164301 (2004).

[34] C. Tietz, S. Schuler, T. Speck, U. Seifert, and J. Wrachtrup, Measurement of Stochastic Entropy Production, Phys. Rev. Lett. 97, 050602 (2006).

[35] K. Hayashi, H. Ueno, R. Iino, and H. Noji, Fluctuation Theorem Applied to F1-ATPase, Phys. Rev. Lett. 104, 218103 (2010).

[36] O.-P. Saira, Y. Yoon, T. Tanttu, M. Möttönen, D. Averin, and J.P. Pekola, Test of the Jarzynski and Crooks Fluctuation Relations in an Electronic System, Phys. Rev. Lett. 109, 180601 (2012).

[37] C. Bustamante, J. Liphardt, and F. Ritort, The Nonequilibrium Thermodynamics of Small Systems, Phys. Today 58, 43 (2005).

[38] C. Jarzynski, Equalities and Inequalities: Irreversibility and the Second Law of Thermodynamics at the Nanoscale, Annu. Rev. Condens. Matter Phys. 2, 329 (2011).

[39] U. Seifert, Stochastic Thermodynamics, Fluctuation Theorems and Molecular Machines, Rep. Prog. Phys. 75, 126001 (2012).

[40] S. R. De Groot and P. Mazur, Non-Equilibrium Thermodynamics (Dover, New York, 1984).

[41] F. Jülicher, A. Ajdari, and J. Prost, Modeling Molecular Motors, Rev. Mod. Phys. 69, 1269 (1997).

[42] M. Esposito, Stochastic Thermodynamics under Coarse Graining, Phys. Rev. E 85, 041125 (2012).

[43] J. L. Doob, Stochastic Processes (John Wiley \& Sons, Chapman \& Hall, New York, 1953).

[44] J. L. Doob, Measure Theory (Springer Science \& Business Media, Berlin, 1994), Vol. 143.

[45] Variables that are even under time reversal do not change sign under time reversal (e.g., position variables), whereas variables that are odd under time reversal change sign under time reversal (e.g., momenta variables or current variables).

[46] C. Maes, On the Origin and the Use of Fluctuation Relations for the Entropy, Séminaire Poincaré 2, 29 (2003).

[47] É. Roldán, I. Neri, M. Dörpinghaus, H. Meyr, and F. Jülicher, Decision Making in the Arrow of Time, Phys. Rev. Lett. 115, 250602 (2015).

[48] P. G. Bergmann and J. L. Lebowitz, New Approach to Nonequilibrium Processes, Phys. Rev. 99, 578 (1955).

[49] G. E. Crooks, Path-Ensemble Averages in Systems Driven Far from Equilibrium, Phys. Rev. E 61, 2361 (2000).

[50] M. Bauer and F. Cornu, Local Detailed Balance: A Microscopic Derivation, J. Phys. A 48, 015008 (2014).

[51] For a system in contact with $\ell$ thermal reservoirs at temperatures $T_{i}, i=1, \ldots, \ell$, the change of environment entropy $S_{\text {env }}(t)$ is equal to $S_{\text {env }}(t)=-\sum_{i=1}^{\ell} Q_{i}(t) / T_{i}$, with $Q_{i}(t)$ the heat exchanged between the $i$-th reservoir and the system.

[52] H. Risken, The Fokker-Planck Equation (Springer, Berlin, 1984).
[53] R. Liptser and A. N. Shiryaev, Statistics of Random Processes: I. General Theory, 2nd ed. (Springer Science \& Business Media, Berlin, 2013), Vol. 5.

[54] H. L. Royden and P. Fitzpatrick, Real Analysis (MacMillan, New York, 1988), Vol. 198.

[55] D. Williams, Probability with Martingales (Cambridge University Press, Cambridge, 1991).

[56] T. Tao, An Introduction to Measure Theory (American Mathematical Society, Providence, 2011), Vol. 126.

[57] In the theory of stochastic processes, one often refers to continuous processes that may have jumps as càdlàg processes (continue à droite, limite à gauche), which are processes that are right continuous everywhere and have left limits everywhere.

[58] C. Maes, F. Redig, and A. Van Moffaert, On the Definition of Entropy Production, via Examples, J. Math. Phys. 41, 1528 (2000).

[59] M. Musiela and M. Rutkowski, Martingale Methods in Financial Modelling (Springer Science \& Business Media, Berlin, 2006), Vol. 36.

[60] T. Björk, Arbitrage Theory in Continuous Time (Oxford University Press, Oxford, 2009).

[61] C. Jarzynski, Nonequilibrium Equality for Free Energy Differences, Phys. Rev. Lett. 78, 2690 (1997).

[62] É. Roldán, I. A. Martínez, J. M. R. Parrondo, and D. Petrov, Universal Features in the Energetics of Symmetry Breaking, Nat. Phys. 10, 457 (2014).

[63] H. Qian and X. S. Xie, Generalized Haldane Equation and Fluctuation Theorem in the Steady-State Cycle Kinetics of Single Enzymes, Phys. Rev. E 74, 010902 (2006).

[64] H. Wang and H. Qian, On Detailed Balance and Reversibility of Semi-Markov Processes and SingleMolecule Enzyme Kinetics, J. Math. Phys. 48, 013303 (2007).

[65] A. B. Kolomeisky, E. B. Stukalin, and A. A. Popov, Understanding Mechanochemical Coupling in Kinesins Using First-Passage-Time Processes, Phys. Rev. E 71, 031902 (2005).

[66] H. Ge, Waiting Cycle Times and Generalized Haldane Equality in the Steady-State Cycle Kinetics of Single Enzymes, J. Phys. Chem. B 112, 61 (2008).

[67] M. Lindén, Decay Times in Turnover Statistics of Single Enzymes, Phys. Rev. E 78, 010901 (2008).

[68] C. Jia, D. Jiang, and M. Qian, Cycle Symmetries and Circulation Fluctuations for Discrete-Time and Continuous-Time Markov Chains, Ann. Appl. Probab. 26, 2454 (2016).

[69] H. Qian, S. Kjelstrup, A. B. Kolomeisky, and D. Bedeaux, Entropy Production in Mesoscopic Stochastic Thermodynamics: Nonequilibrium Kinetic Cycles Driven by Chemical Potentials, Temperatures, and Mechanical Forces, J. Phys. Cond. Mat. 28, 153004 (2016).

[70] M. Bauer and F. Cornu, Affinity and Fluctuations in a Mesoscopic Noria, J. Stat. Phys. 155, 703 (2014).

[71] M. Dörpinghaus, É. Roldán, I. Neri, H. Meyr, and F. Jülicher, An Information Theoretic Analysis of Sequential Decision-Making, arXiv:1510.08952.

[72] H. A. Kramers, Brownian Motion in a Field of Force and the Diffusion Model of Chemical Reactions, Physica 7, 284 (1940). 
[73] P. Reimann, Brownian Motors: Noisy Transport Far from Equilibrium, Phys. Rep. 361, 57 (2002).

[74] P. Hänggi and F. Marchesoni, Artificial Brownian Motors: Controlling Transport on the Nanoscale, Rev. Mod. Phys. 81, 387 (2009).

[75] V. Blickle, T. Speck, U. Seifert, and C. Bechinger, Characterizing Potentials by a Generalized Boltzmann Factor, Phys. Rev. E 75, 060101 (2007).

[76] J. R. Gomez-Solano, A. Petrosyan, S. Ciliberto, R. Chetrite, and K. Gawedzki, Experimental Verification of a Modified Fluctuation-Dissipation Relation for a MicronSized Particle in a Nonequilibrium Steady State, Phys. Rev. Lett. 103, 040601 (2009).

[77] J. R. Gomez-Solano, A. Petrosyan, S. Ciliberto, and C. Maes, Fluctuations and Response in a Non-Equilibrium Micron-Sized System, J. Stat. Mech. (2011) P01008.

[78] K. Saito and A. Dhar, Waiting for Rare Entropic Fluctuations, Europhys. Lett. 114, 50004 (2016).

[79] A. Gomez-Marin and I. Pagonabarraga, Test of the Fluctuation Theorem for Stochastic Entropy Production in a Nonequilibrium Steady State, Phys. Rev. E 74, 061113 (2006).

[80] I. I. Gichman and A. W. Skorochod, Stochastische Differentialgleichungen (Akademie-Verlag, Berlin, 1971), Chap. 3, pp. 100-108.

[81] W. C. Lindsey and H. Meyr, Complete Statistical Description of the Phase-Error Process Generated by Correlative Tracking Systems, IEEE Trans. Inf. Theory 23, 194 (1977).

[82] D. Keller and C. Bustamante, The Mechanochemistry of Molecular Motors, Biophys. J. 78, 541 (2000).

[83] P. Hänggi, P. Talkner, and M. Borkovec, Reaction-Rate Theory: Fifty Years After Kramers, Rev. Mod. Phys. 62, 251 (1990).

[84] S. Toyabe, T. Watanabe-Nakayama, T. Okamoto, S. Kudo, and E. Muneyuki, Thermodynamic Efficiency and Mechanochemical Coupling of F1-ATPase, Proc. Natl. Acad. Sci. U.S.A. 108, 17951 (2011).

[85] E. A. Galburt, S. W. Grill, A. Wiedmann, L. Lubkowska, J. Choy, E. Nogales, M. Kashlev, and C. Bustamante, Backtracking Determines the Force Sensitivity of RNAP II in a Factor-Dependent Manner, Nature 446, 820 (2007).

[86] M. Depken, E. A. Galburt, and S. W. Grill, The Origin of Short Transcriptional Pauses, Biophys. J. 96, 2189 (2009).

[87] F. Jülicher and R. Bruinsma, Motion of RNA Polymerase Along DNA: A Stochastic Model, Biophys. J. 74, 1169 (1998).

[88] N. Komissarova and M. Kashlev, Transcriptional Arrest: Escherichia coli RNA Polymerase Translocates Backward, Leaving the 3' End of the RNA Intact and Extruded, Proc. Natl. Acad. Sci. U.S.A. 94, 1755 (1997).

[89] Another mechanism of backtrack recovery is cleavage of the backtracked RNA. Cleavage can be performed intrinsically by the polymerase itself or with the help of the TFIIS "transcription factor." In yeast, for polymerase I, cleavage plays an important role [90], but for polymerase II, the cleavage activity is negligible with respect to diffusion during backtrack recovery. For simplicity, we do not consider cleavage here.

[90] A. Lisica, C. Engel, M. Jahnel, É. Roldán, E. A. Galburt, P. Cramer, and S. W. Grill, Mechanisms of Backtrack Recovery by RNA Polymerases I and II, Proc. Natl. Acad. Sci. U.S.A. 113, 2946 (2016).

[91] B. Zamft, L. Bintu, T. Ishibashi, and C. Bustamante, Nascent RNA Structure Modulates the Transcriptional Dynamics of RNA Polymerases, Proc. Natl. Acad. Sci. U.S.A. 109, 8948 (2012).

[92] T. Ishibashi, M. Dangkulwanich, Y. Coello, T. A. Lionberger, L. Lubkowska, A. S. Ponticelli, M. Kashlev, and C. Bustamante, Transcription Factors IIS and IIF Enhance Transcription Efficiency by Differentially Modifying RNA Polymerase Pausing Dynamics, Proc. Natl. Acad. Sci. U.S.A. 111, 3419 (2014).

[93] M. Dangkulwanich, T. Ishibashi, S. Liu, M. L. Kireeva, L. Lubkowska, M. Kashlev, and C. J. Bustamante, Complete Dissection of Transcription Elongation Reveals Slow Translocation of RNA Polymerase II in a Linear Ratchet Mechanism, eLife 2, e00971 (2013).

[94] M. Depken, J. M. R. Parrondo, and S. W. Grill, Intermittent Transcription Dynamics for the Rapid Production of Long Transcripts of High Fidelity, Cell Reports 5, 521 (2013).

[95] M. H. Larson, J. Zhou, C. D. Kaplan, M. Palangat, R. D. Kornberg, R. Landick, and S. M. Block, Trigger Loop Dynamics Mediate the Balance between the Transcriptional Fidelity and Speed of RNA Polymerase II, Proc. Natl. Acad. Sci. U.S.A. 109, 6555 (2012).

[96] Note that here we use passage probabilities of entropy production with a given initial state as discussed in Appendix D.

[97] The bound holds despite the presence of an absorbing boundary since the effect of this boundary is to reduce the maximum depth of the backtrack.

[98] A. C. Barato and U. Seifert, Thermodynamic Uncertainty Relation for Biomolecular Processes, Phys. Rev. Lett. 114, 158101 (2015).

[99] T. R. Gingrich, J. M. Horowitz, N. Perunov, and J. L. England, Dissipation Bounds All Steady-State Current Fluctuations, Phys. Rev. Lett. 116, 120601 (2016).

[100] P. Pietzonka, A. C. Barato, and U. Seifert, Universal Bounds on Current Fluctuations, Phys. Rev. E 93, 052145 (2016).

[101] M. Polettini, A. Lazarescu, and M. Esposito, Tightening the Uncertainty Principle for Stochastic Currents, Phys. Rev. E 94, 052104 (2016).

[102] S. N. Majumdar and P. Krapivsky, Extreme Value Statistics and Traveling Fronts: Various Applications, Physica A 318, 161 (2003).

[103] A. Comtet and S. N. Majumdar, Precise Asymptotics for a Random Walker's Maximum, J. Stat. Mech. (2005) P06013.

[104] S. N. Majumdar and A. Pal, Extreme Value Statistics of Correlated Random Variables, arXiv:1406.6768.

[105] P. Mestres, I. A. Martínez, A. Ortiz-Ambriz, R. A. Rica, and É. Roldán, Realization of Nonequilibrium Thermodynamic Processes Using External Colored Noise, Phys. Rev. E 90, 032116 (2014). 
[106] Y. Jun, M. Gavrilov, and J. Bechhoefer, High-Precision Test of Landauer's Principle in a Feedback Trap, Phys. Rev. Lett. 113, 190601 (2014).

[107] M. Nishiyama, H. Higuchi, and T. Yanagida, Chemomechanical Coupling of the Forward and Backward Steps of Single Kinesin Molecules, Nat. Cell Biol. 4, 790 (2002).

[108] D. Collin, F. Ritort, C. Jarzynski, S. B. Smith, I. Tinoco, and C. Bustamante, Verification of the Crooks Fluctuation Theorem and Recovery of RNA Folding Free Energies, Nature 437, 231 (2005).

[109] M. V. Bonança and C. Jarzynski, Conditional Reversibility in Nonequilibrium Stochastic Systems, Phys. Rev. E 93, 022101 (2016).

[110] A. Tartakovsky, I. Nikiforov, and M. Basseville, Sequential Analysis: Hypothesis Testing and Changepoint Detection (CRC Press, Boca Raton, 2014).

[111] M. Morse, J. Bell, G. Li, and J. X. Tang, Flagellar Motor Switching in Caulobacter Crescentus Obeys First Passage Time Statistics, Phys. Rev. Lett. 115, 198103 (2015).

[112] P. Sartori, L. Granger, C. F. Lee, and J. M. Horowitz, Thermodynamic Costs of Information Processing in Sensory Adaptation, PLoS Comput. Biol. 10, e1003974 (2014).
[113] A. C. Barato, D. Hartich, and U. Seifert, Efficiency of Cellular Information Processing, New J. Phys. 16, 103024 (2014).

[114] S. Bo, M. Del Giudice, and A. Celani, Thermodynamic Limits to Information Harvesting by Sensory Systems, J. Stat. Mech. (2015) P01014.

[115] S. G. Das, G. Iyengar, and M. Rao, A Lower Bound on the Free Energy Cost of Molecular Measurements, arXiv: 1608.07663.

[116] J. L. England, Statistical Physics of Self-Replication, J. Chem. Phys. 139, 121923 (2013).

[117] P. Sartori and S. Pigolotti, Thermodynamics of Error Correction, Phys. Rev. X 5, 041039 (2015).

[118] T. J. Kobayashi, Implementation of Dynamic Bayesian Decision Making by Intracellular Kinetics, Phys. Rev. Lett. 104, 228104 (2010).

[119] E. D. Siggia and M. Vergassola, Decisions on the Fly in Cellular Sensory Systems, Proc. Natl. Acad. Sci. U.S.A. 110, E3704 (2013).

[120] J. Wang, Landscape and Flux Theory of Non-Equilibrium Dynamical Systems with Application to Biology, Adv. Phys. 64, 1 (2015).

[121] I. Marzuoli, Master thesis, 2015.

[122] S. Redner, A Guide to First-Passage Processes (Cambridge University Press, Cambridge, 2001).

[123] S. Sato and J. Inoue, Inverse Gaussian Distribution and Its Application, Electron. Comm. Jpn. 77, 32 (1994). 